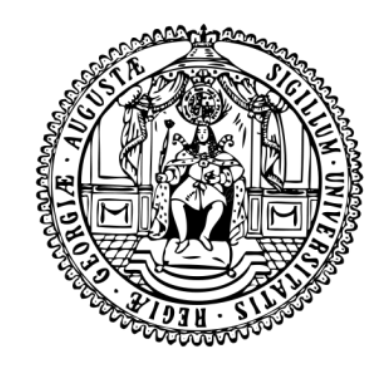

\title{
Cardiac functions of the cellular oxygen sensors prolyl-4-hydroxylase domain enzymes 2 and 3
}

\author{
Kumulative Dissertation \\ zur Erlangung des mathematisch-naturwissenschaftlichen Doktorgrades \\ "Doctor rerum naturalium" \\ der Georg-August-Universität zu Göttingen
}

vorgelegt von

Marion Hölscher

aus Paderborn

Göttingen 2012 

Mitglieder des Betreuungsauschusses:

Referentin: Prof. Dr. S. Pöggeler

Koreferentin: Prof. Dr. F. Alves

Koreferentin: Prof. Dr. D. M. Katschinski

Tag der mündlichen Prüfung: 06.06.2012 


\section{Statutory Declaration}

I declare that I have authored this thesis independently, that I have not used other than the declared sources / resources, and that I have explicitly marked all material which has been quoted either literally or by content from the used sources.

Marion Hölscher 


\section{Contents}

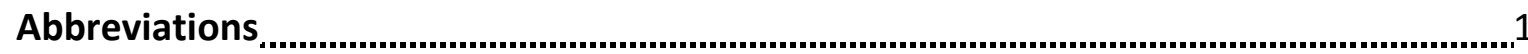

List of publications

1. Summary

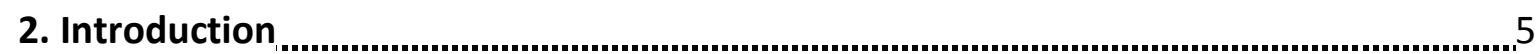

2.1 Hypoxia

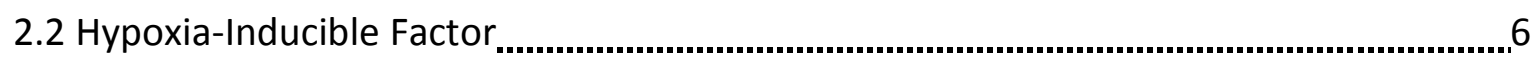

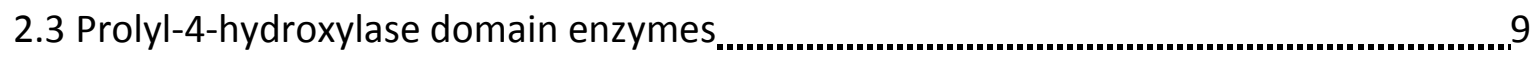

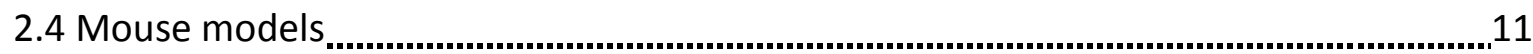

2.4.1 PHD knock-out mice

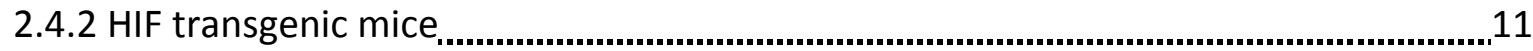

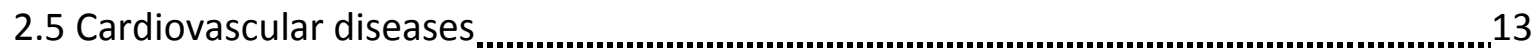

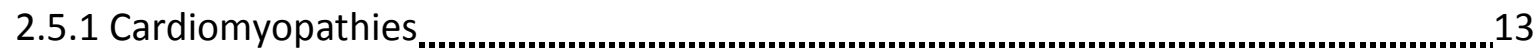

2.5.2 Mechanical load: preload versus afterload ............................................................13

2.5.2 Myocardial infarction ................................................................................................

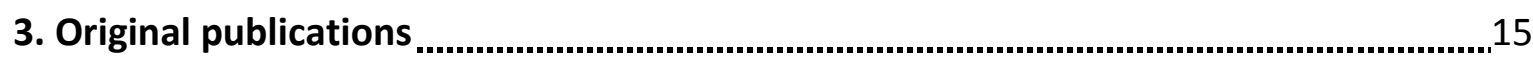

3.1 Cardiomyocyte-specific Prolyl-4-hydroxylase Domain 2 Knock Out Protects from

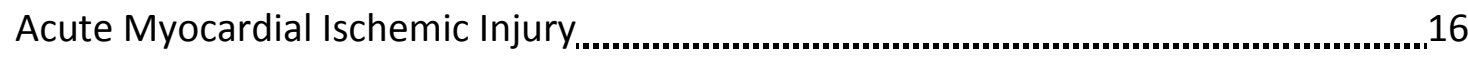

3.2 Unfavourable consequences of chronic cardiac HIF-1 $\alpha$ stabilization .............................28

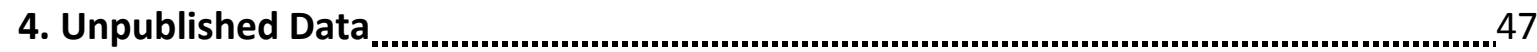

4.1 Detrimental consequences of a cardiomyocyte-specific knock-out of PHD2 .................47

4.2 Cardiac-specific double knock-out of PHD2 and PHD3 leads to dilated cardiomyopathy and a premature death................................................................. 50

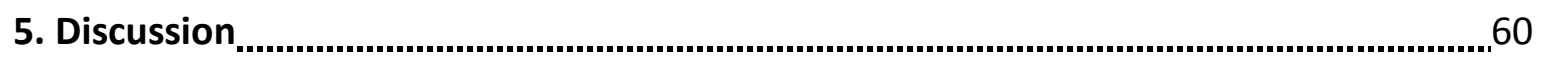

5.1 Beneficial effects of PHD2 knock-out, respectively HIF-1 $\alpha$ stabilization .........................61

5.2 Detrimental effects of PHD2 knock-out, respectively HIF-1 $\alpha$ stabilization ......................62

5.3 Cardiomyocyte-specific PHD2/PHD3 double knock-out mice ........................................64

5.4 PHD inhibitors as a therapeutic approach in acute myocardial infarction .....................65

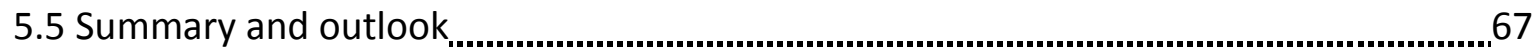

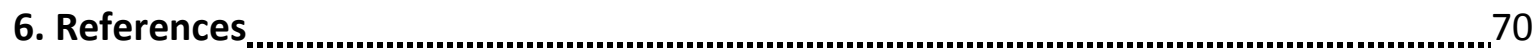

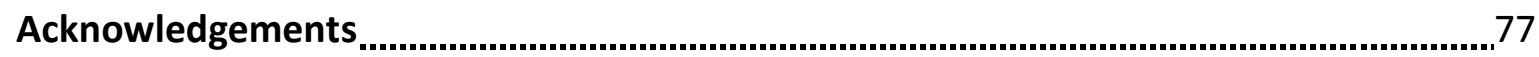

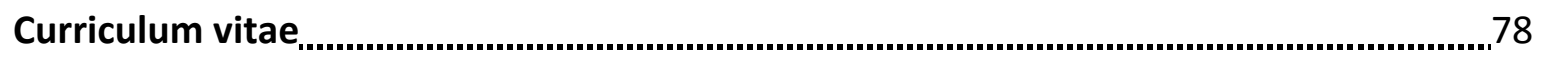


Abbreviations

\begin{tabular}{|c|c|}
\hline 2-OG & 2-oxoglutarate \\
\hline$\alpha \mathrm{MHC}$ & $\alpha$-myosin heavy chain \\
\hline AAR & area at risk \\
\hline AMPK & 5'-adenosine monophosphate-activated protein kinase \\
\hline AON & area of necrosis \\
\hline ARNT & aryl hydrocarbon nuclear translocator \\
\hline bHLH & basic helix-loop-helix \\
\hline $\mathrm{Ca}^{2+}$ & calcium \\
\hline CA IX & carbonic anhydrase 9 \\
\hline CSQ & calsequestrin \\
\hline C-TAD & C-terminal transactivation domain \\
\hline DCM & dilated cardiomyopathy \\
\hline DMOG & dimethyloxaloylglycine \\
\hline $\mathrm{E}$ & embryonic day \\
\hline e.g. & exempli gratia (for example) \\
\hline EGLN & egg-laying deficiency protein nine-like protein \\
\hline Fig & figure \\
\hline $\mathrm{FIH}-1$ & factor inhibiting HIF-1 \\
\hline FAS & fractional area shortening \\
\hline FDG & fluordesoxyglucose \\
\hline $\mathrm{Fe}^{2+}$ & iron \\
\hline FS & fractional shortening \\
\hline FTHA & fluoro-6-thia-heptadecanoic acid \\
\hline Glut1 & glucose transporter 1 \\
\hline H9c2 & rat heart cell-line \\
\hline HCM & hypertrophic cardiomyopathy \\
\hline HIF & hypoxia-inducible factor \\
\hline HRE & hypoxia responsive element \\
\hline ICM & ischemic cardiomyopathy \\
\hline i.e. & id est (that is) \\
\hline LAD & left anterior descending artery \\
\hline LVEDD & left ventricular end-diastolic diameter \\
\hline LVESD & left ventricular end-systolic diameter \\
\hline $\mathrm{Ml}$ & myocardial infarction \\
\hline $\mathrm{O}_{2}$ & oxygen \\
\hline ODDD & oxygen-dependent degradation domain \\
\hline PAS & PER-ARNT-SIM \\
\hline PET & positron emission tomography \\
\hline Pfkl & phosphofructokinase I \\
\hline PHD & prolyl -4-hydroxylase domain enzyme \\
\hline PLB & phospholamban \\
\hline RyR2 & type- 2 ryanodine receptor \\
\hline SEM & standard error of the mean \\
\hline SERCA & sarcoplasmic/endoplasmic reticulum calcium ATPase \\
\hline SUV & standard uptake volume \\
\hline TAC & transverse aortic constriction \\
\hline TTC & triphenyltetrazolium chloride \\
\hline pVHL & von Hippel-Lindau protein \\
\hline qRT-PCR & quantitative real-time polymerase chain reaction \\
\hline & wild-type \\
\hline
\end{tabular}




\section{List of Publications}

Marion Hölscher, Monique Silter, Sabine Krull, Melanie von Ahlen, Amke Hesse, Peter Schwartz, Ben Wielockx, Georg Breier, Dörthe M. Katschinski, Anke Zieseniss (2011)

Cardiomyocyte-specific Prolyl-4-hydroxylase Domain 2 Knock Out Protects from Acute Myocardial Ischaemic Injury

J Biol Chem 286:11185-11194

Marion Hölscher, Katrin Schäfer, Sabine Krull, Katja Farhat, Amke Hesse, Monique Silter, Yun Lin, Bernd Pichler, Patricia Thistlethwaite, Ali El-Armouche, Lars S. Maier, Dörthe M. Katschinski, Anke Zieseniss (2012)

Unfavourable consequences of chronic cardiac HIF-1 $\alpha$ stabilization

Cardiovasc Res. 94:77-86 


\section{Summary}

\section{Summary}

Cardiovascular diseases are the major cause of death in the western world and produce furthermore the highest medical costs among all diseases. About $41 \%$ of all deaths in Germany in the year 2010 can be attributed to cardiovascular diseases ("Statistisches Bundesamt Deutschland", press release no. 354, 23.09.2011). About $17 \%$ of these deaths were caused by myocardial infarction (men: $56 \%$, women: $44 \%$ ). Cardiac tissue protection in case of acute ischemia could therefore result in better short and long term survival of these patients.

In 2003 it was described for the first time that the Hypoxia-inducible factor (HIF), a heterodimeric transcription factor, which is stabilized under hypoxic conditions, e.g. in myocardial infarction, can mediate cardioprotection (Cai et al., 2003). It was shown that the cardioprotective effect of intermittent hypoxia, which is defined as repeated episodes of hypoxia interspersed with episodes of normoxia, is dependent on the HIF-1 $\alpha$ gene dosage. This was shown by a better ventricular contractile function and a significantly smaller infarct size following ischemia-reperfusion in mice pretreated with intermittent hypoxia. This preconditioning effect was lost in HIF-1 $\alpha^{+/-}$mice. Since then a lot of subsequent papers have been published that demonstrate the crucial role for HIF-1 in various modalities of cardioprotection (e.g. Kido et al., 2005; Date et al. 2005). With the discovery of the HIF regulating proly-4 hydroxylase domain (PHD) enzymes 1-3 potential therapeutic targets to modulate HIF-1 $\alpha$ stability and thus possibilities to improve ischemic diseases have been discovered. Despite these findings a lot of open questions remain that will be addressed in my PhD thesis.

- Are there any effects of long-term stabilization of HIF-1 $\alpha$ in the heart?

- Are there any PHD-dependent but HIF-independent effects that need to be considered if PHD inhibiting drugs are being developed in order to stabilize HIF-1 $\alpha$ ?

- Is HIF-1 $\alpha$ stabilization also beneficial after elevated mechanical load?

- Are there isoform specific functions of the three PHDs that need to be taken into account when designing PHD inhibitors?

- Which cell parameters like calcium handling or metabolism are modulated by HIF-1 $\alpha$ stabilization that could be beneficial or detrimental for the heart? 


\section{Summary}

To answer some of the above mentioned questions I have analyzed several genetically modified mouse strains during my thesis:

-cardiomyocyte-specific PHD2 knock-out mice (cPhd $\left.2^{-/}\right)$

-cardiac-specific transgenic HIF-1 $\alpha$ mice $\left(H i f-1 \alpha^{t g}\right)$

-cardiomyocyte-specific PHD2/PHD3 double knock-out mice (cPhd2/3/-)

In summary, I was able to generate cardiomyocyte-specific PHD2 knock-out mice (cPhd $2^{-/}$) with subsequent mild stabilization of HIF-1 $\alpha$. These mice are protected from acute myocardial infarction as shown by a smaller area at risk (AAR) and a smaller area of necrosis (AON) six hours after ligation of the left anterior descending artery (LAD). Three weeks after the ligation these mice show a better preserved heart function compared to $w t$ littermates. This phenomenon is at least in part due to an increased capillary area in the hearts of $\mathrm{CPhd}^{-/}$mice. When challenged by elevated mechanical load by performing transverse aortic constriction (TAC) surgery these mice show a comparable degree of hypertrophy with a similar decline in heart function. Aging $\mathrm{CPhd} 2^{-/-}$mice develop a mild hypertrophy which does not occur in wt mice.

Furthermore I could show in a cardiac-specific HIF-1 $\alpha$ transgenic mouse model $\left(H i f-1 \alpha^{\text {tg }}\right)$ that the constitutive expression of HIF-1 $\alpha$ leads to changes in capillary area and shifts the cardiac metabolism towards glycolysis. Furthermore, calcium- $\left(\mathrm{Ca}^{2+}\right)$ handling of the cells is altered, which leads to increased $\mathrm{Ca}^{2+}$ transients and a faster intracellular $\mathrm{Ca}^{2+}$ decline. After TAC surgery Hif- $1 \alpha^{\text {tg }}$ mice show a strong cardiac decompensation. Moreover, cardiomyopathy was also seen in aging $H i f-1 \alpha^{t g}$ mice.

Preliminary data of cardiomyocyte-specific PHD2/PHD3 double knock-out mice ( $c$ Phd $\left.2 / 3^{-/-}\right)$ illustrate detrimental effects with severe dilated cardiomyopathy that lead to a premature death at the age of 4.5 to 6 months.

Summing up, I could show that a short-term stabilization of HIF-1 $\alpha$ seems to have beneficial effects on the heart whereas long-term stabilization seems to be detrimental. 


\section{Introduction}

\section{Introduction}

\subsection{Hypoxia}

The maintenance of cellular oxygen homeostasis is critical to all aerobic organisms in order to e.g. produce energy for cell survival, motility or reproduction. In case of reduced oxygen availability hypoxia develops. Hypoxia is a condition in which the whole body (generalized hypoxia) or parts of the body (tissue hypoxia) are deprived of an adequate oxygen supply. This is due to an imbalance of oxygen availability and oxygen consumption. Anoxia is defined by a complete lack of oxygen. Hypoxia occurs physiologically during embryonic development (3-5\% $\left.\mathrm{O}_{2}\right)$ when hypoxia is required for vascular growth, which is in turn important for successful development (Asikainen and White, 2007), or when ascending to higher altitudes (Hackett and Roach, 2001). This can cause high-altitude sickness leading to life-threatening conditions (Paralikar and Paralikar, 2010).

Tissue hypoxia occurs when there is a local restriction of the blood flow to otherwise welloxygenated tissues. The reduced oxygen supply is then insufficient to satisfy the tissue's oxygen demand. This pathophysiological hypoxia occurs for example in ischemic diseases or in solid tumors.

The master regulator of the cellular hypoxia response pathway is the transcription factor hypoxia-inducible factor (HIF). HIF was discovered by the identification of a hypoxia response element (HRE) (5'-RCGTG-3') in the enhancer of the erythropoietin gene (Goldberg et al., 1988; Semenza, 1998), a glycoprotein hormone that controls erythropoiesis. In following studies HIF was identified as a heterodimeric transcription factor consisting of an oxygensensitive $\alpha$-subunit and a constitutively expressed $\beta$-subunit, which was beforehand identified as the aryl hydrocarbon nuclear translocator (ARNT), a binding partner of the aryl hydrocarbon receptor (Reyes et al., 1992).

Among the critical physiological processes regulated by HIF-1 are erythropoiesis, angiogenesis, and glycolysis, which are examples of systemic, local tissue, and intracellular adaptive responses to hypoxia. So far, more than 200 genes were identified to be HIFdependently regulated (Benita et al., 2009; Ortiz-Barahona et al., 2010), but it is assumed that in total about $2 \%$ of the human genome is hypoxia-inducible. 


\section{Introduction}

\subsection{Hypoxia-inducible factor}

HIFs are heterodimeric transcription factors consisting of one out of three oxygen-sensitive $\alpha$-subunits (HIF- $1 \alpha$, HIF- $2 \alpha$ or HIF-3 $\alpha$ ) and the common oxygen-insensitive HIF- $\beta$ subunit. Both subunits belong to the PER-ARNT-SIM (PAS) subfamily of the basic helix-loop-helix (bHLH) family of transcription factors. The N-terminal domain contains the bHLH and the PAS domains which facilitate the dimerization of the HIF- $\alpha$ and the HIF- $\beta$ subunit and allows the binding to hypoxia responsive elements (HREs) of HIF target genes (Erbel et al., 2003).

The $\mathrm{N}$ - and the C-terminal oxygen-dependent degradation domains (ODDD) are located within the $\mathrm{N}$-terminal transactivation domain. The $\mathrm{O}_{2^{-}}, \mathrm{Fe}^{2+}-, 2$-oxoglutarate- (2-OG) and ascorbate-dependent proly-4-hydroxylase domain (PHD) proteins, PHD1, PHD2 and PHD3, can hydroxylate two proline residues ( Pro $^{402}$ and $\mathrm{Pro}^{564}$ ) within this domain and thereby influence the stability of HIF- $\alpha$. The hydroxylation initiates the binding of an E3 ubiquitin ligase, the von Hippel-Lindau ( $\mathrm{VVHL}$ ) protein. $\mathrm{pVHL}$ is a component of a protein-ubiquitin ligase complex that targets the $\alpha$-subunit for rapid degradation by the proteasome (Maxwell et al., 1999).

Besides the HIF- $\alpha$ stability also the HIF- $\alpha$ activity is determined by hydroxylation. In 2001 the factor inhibiting HIF (FIH-1) was first described (Mahon et al., 2001). It can hydroxylate an asparagine residue $\left(\mathrm{Asn}^{803}\right)$ in the C-terminal transactivation domain (C-TAD) of HIF-1 $\alpha$. Through this hydroxylation the binding of necessary co-factors, such as p300, is blocked and so the transcriptional activation of HIF-1 is restricted (Lando et al., 2002).

In figure 1 a schematic drawing of HIF-1 $\alpha$ and the oxygen-dependent regulation of HIF-1 $\alpha$ protein stabilization and activation are shown. 


\section{Introduction}

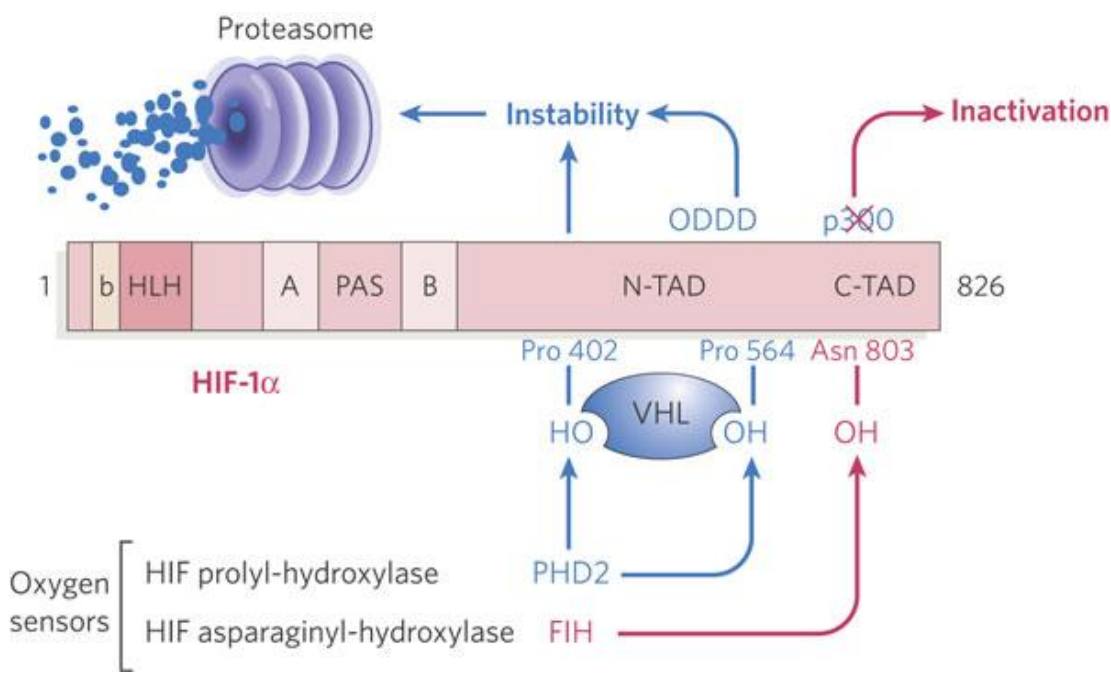

Taken from Pouysségur et al. (2006)

Fig.1 Schematic drawing of HIF-1 $\alpha$ and the oxygen-dependent regulation of HIF-1 $\alpha$ protein stabilization and activation. The basic helix-loop-helix (bHLH) domain, involved in DNA-binding, and the Per-Arnt-Sim (PAS) A and B domains, which are responsible for heterodimerization with HIF-1B, are located in the N-terminal region of HIF-1 $\alpha$. The proline residues $\operatorname{Pro}^{402}$ and Pro $^{564}$ that are hydroxylated by the PHD enzymes are located in the oxygen-dependent degradation domain (ODDD). The factor inhibiting HIF (FIH) can hydroxylate an asparagine residue $\left(\mathrm{Asn}^{803}\right)$ in the C-terminal transcriptional activation domain (C-TAD) of HIF-1 $\alpha$ and inhibits thereby the binding of cofactors, such as p300, that are required for the transcription of HIF-dependent genes. The N-TAD, a second transcriptional activation domain, overlapping with the ODDD, is $\mathrm{FIH}$-independent and may be involved in distinct gene expression.

In normoxia the transcriptionally inactive HIF-1 $\alpha$ subunit is quickly degraded with a half-life of less than five minutes. In hypoxia on the contrary, the oxygen dependent PHDs and FIH are enzymatically inactive and hence cannot hydroxylate the proline and the asparagine residues of the HIF-1 $\alpha$ subunit. Thereupon, the non-degraded, stabilized HIF-1 $\alpha$ accumulates into the nucleus where it dimerizes with the constitutively expressed HIF-1ß subunit. Subsequently HIF-1 binds to HREs of HIF target genes, e.g. Glut1 or VEGF, which are then transcriptionally activated (see fig. 2). 


\section{Introduction}

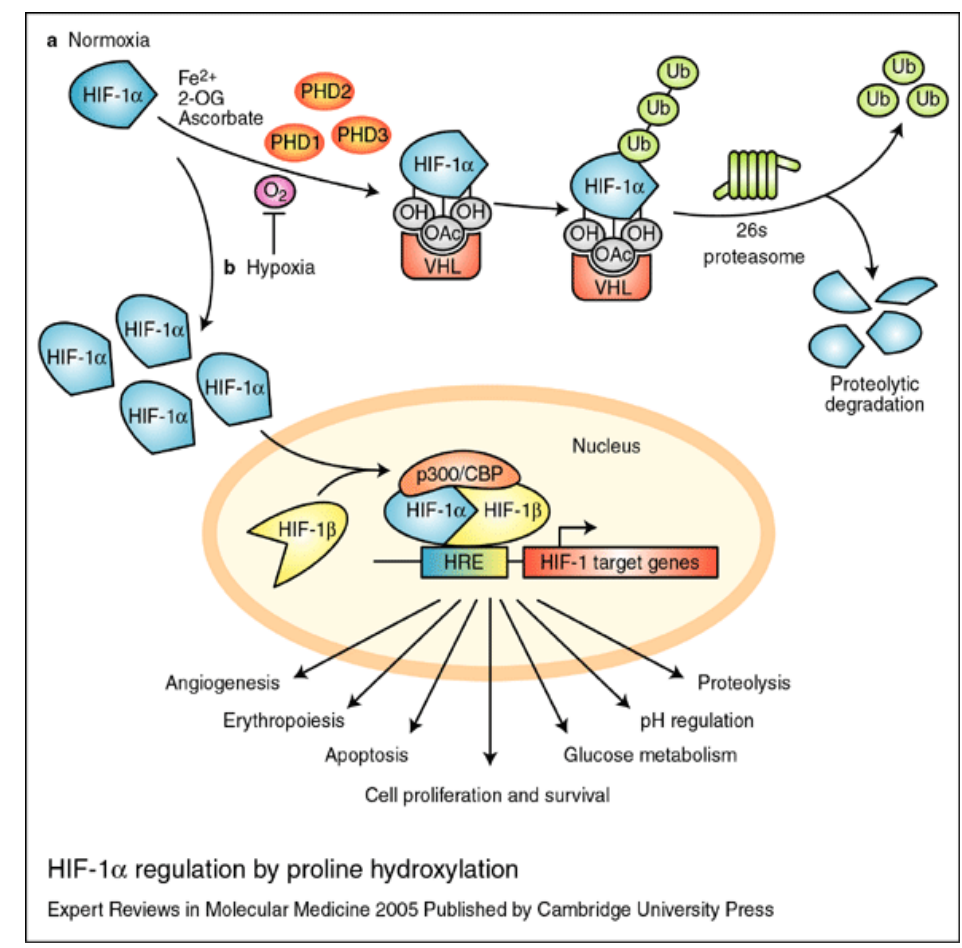

Taken from Carroll et al. (2005)

Fig.2 HIF-1 $\alpha$ in hypoxia and normoxia. Under normoxic conditions HIF-1 $\alpha$ is hydroxylated by the PHDs. This hydroxylation triggers its association with the PVHL E3 ligase complex which leads to rapid proteasomal degradation of HIF-1 $\alpha$. In hypoxia the oxygen-dependent PHDs cannot hydroxylate the HIF- $1 \alpha$ subunit. HIF- $1 \alpha$ is consequently stabilized and translocates into the nucleus where it dimerizes with HIF-1 $\beta$. After binding to p300/CBP the transcription of HIF-target genes, like Glut1 or VEGF, is induced.

HIF-1 has been detected in all metazoan species that have been analyzed from Trichoplax adhaerens (Loernaz et al., 2011) to Homo sapiens, suggesting that the appearance of HIF-1 represented an adaptation that was essential to metazoan evolution.

After the discovery of HIF-1 $\alpha$ two additional HIF- $\alpha$ isoforms were identified - HIF- $2 \alpha$ and HIF-3 $\alpha$ (Ema et al., 1997; Flamme et al., 1997). Both HIF-2 $\alpha$ and HIF-3 $\alpha$ display close sequence similarity to HIF-1 $\alpha$ in the N-terminal part of the 22 polypeptide containing the bHLH and PAS domains, but HIF-3 $\alpha$ lacks the C-TAD (Ema et al., 1997; Flamme et al., 1997; Gu et al., 1998; Hogenesch et al., 1997; Tian et al., 1997.)

HIF- $1 \alpha$ and HIF- $2 \alpha$ have a number of structural and biochemical similarities and both bind to HREs. But in contrast to the ubiquitously expressed HIF-1 $\alpha$, HIF-2 $\alpha$ is mainly expressed in the lung, endothelium and carotid body (Ema et al., 1997; Tian et al., 1998). Furthermore there is evidence that HIF-1 $\alpha$ preferentially activates genes that are important for glycolysis, while HIF-2 $\alpha$ appears to favor the activation of genes involved in angiogenesis (Hu et al., 2003). The importance of HIF- $1 \alpha$ and HIF- $2 \alpha$ can be demonstrated by the fact that knock-out of HIF- 


\section{Introduction}

$1 \alpha$ as well as HIF-2 $\alpha$ leads to embryonic lethality in mice (Compernolle et al., 2002; Iver et al., 1998).

Although less is known about HIF-3 $\alpha$, it could be shown that HIF-3 $\alpha$ can also dimerize with HIF-1 $\beta$ and bind to HREs thereby preventing the binding of HIF-1 and HIF-2. There are splice variants of HIF-3 $\alpha$ without transactivation domains. These variants instead bind to HIF-1 $\alpha$ and prevent its DNA-binding. So in general HIF-3 $\alpha$ acts as a negative regulator of HIF-1 $\alpha$ and HIF-2 $\alpha$ (Hara et al., 2001).

\subsection{Prolyl-4-hydroxylase domain enzymes}

The prolyl-4-hydroxylase domain enzymes (PHDs) have been first described in 2001-2002 as a subfamily of 2-oxoglutarate-dependent dioxygenases that are responsible for the hydroxylation of HIF-1 $\alpha$ (Bruick et al., 2001; Epstein et al., 2002). In lower animals, like nematodes, only a single PHD isoform exists whereas in higher animals, like in mammals, three PHDs (PHD1, PHD2 and PHD3) have been discovered. Another isoform, prolyl-4hydroxylase transmembrane (P4H-TM) was detected but so far the function of this transmembrane protein remains unclear (Oehme et al., 2002; Koivunen et al., 2007).

The PHD enzymes are given several names: prolyl-4-hydroxylase domain enzymes (PHD1, PHD2 and PHD3), HIF-prolyl hydroxylases (HIF-P4H-3, HIF-P4H-2 and HIF-P4H-1) and EGLNs (EGLN-2, EGLN-1 and EGLN-3, respectively). The EGLN terminology refers to the relationship of these proteins to the protein egg-laying-deficient 9 of the nematode worm Caenorhabditis elegans and the term prolyl-hydroxylase domain (PHD) reflects the functionality as hydroxylases but avoids it to consider the PHDs to be HIF-specific as HIF-independent functions of the PHDs have been described (Cummins et al., 2006).

The ability of the PHDs to function as oxygen sensors can be revealed by their $\mathrm{Km}$ values (230- $250 \mu \mathrm{M}$ ) which are slightly above the atmospheric partial oxygen pressure at sea level (Hirsilä et al., 2003). Therefore even small changes in atmospheric oxygen concentration affect the enzymatic function of the PHDs and consequently the transcriptional activity of HIF is induced.

As mentioned before, PHDs hydroxylate two proline residues in HIF's Leu-Xaa-Xaa-Leu-AlaPro motif. It was shown that the hydroxylation activity of PHD2 and PHD3 is similar whereas that of PHD1 is lower (Tuckerman et al., 2004). In this hydroxylation reaction one oxygen atom of the molecular oxygen is added to a peptidyl proline to form hydroxyproline and the 


\section{Introduction}

other oxygen atom is used in a coupled decarboxylation reaction that converts 2-oxoglutarate to succinate; thus, PHDs require 2-oxoglutarate as a co-substrate (McDonough et al., 2006).

PHD2 is clearly the most abundant of the three mammalian isoenzymes and it is also responsible for setting the normoxic steady-state levels of HIF- $\alpha$ (Appelhoff et al., 2004; Berra et al., 2003). Furthermore, PHD2 knock-out mice die between embryonic day (E) 12.5 and 14.5, whereas PHD1 and PHD3 knock-out mice are viable.

The phd 2 and phd3 genes contain HREs which makes these two isoforms inducible by HIF-1 under hypoxia (Metzen et al., 2005; Pescador et al., 2005). This is to guarantee a quick degradation of HIF-1 $\alpha$ after reoxygenation.

Although the PHDs have many features in common, they show some differences in their expression pattern, catalytic properties and physiological role. In table 1 alternative names, the tissue distribution, intracellular localization, substrate preference, hypoxia inducibility, chromosome localization, protein size and the $K \mathrm{~m}$ values are listed.

Table 1 Overview of some features of PHD1, PHD2 and PHD3

\begin{tabular}{|c|c|c|c|}
\hline & PHD1 & PHD2 & PHD3 \\
\hline alternative names & EGLN2, HPH3 [1] & EGLN1, HPH2 [1] & EGLN3, HPH1 [1] \\
\hline $\begin{array}{l}\text { tissue of expression } \\
\text { (in mice) }\end{array}$ & $\begin{array}{l}\text { testis, liver, kidney, } \\
\text { brain, (heart) [2] }\end{array}$ & $\begin{array}{c}\text { heart, liver, brain, } \\
\text { kidney, skeletal muscle, } \\
\text { lung [2] }\end{array}$ & $\begin{array}{c}\text { heart, liver, brain, } \\
\text { kidney, skeletal muscle, } \\
\text { lung [2] }\end{array}$ \\
\hline $\begin{array}{l}\text { intracellular } \\
\text { localization }\end{array}$ & nucleus [5] & cytoplasm [5] & $\begin{array}{l}\text { cytoplasm and } \\
\text { nucleus [5] }\end{array}$ \\
\hline $\begin{array}{l}\text { relative substrate } \\
\text { preference }\end{array}$ & $\begin{array}{l}\text { HIF- } 2 \alpha>\mathrm{HIF}-1 \alpha[3] \\
\text { C-ODD>N-ODD [4] }\end{array}$ & $\begin{array}{l}\text { HIF- } 1 \alpha>\mathrm{HIF}-2 \alpha[3] \\
\text { C-ODD }>\mathrm{N}-\mathrm{ODD}[4]\end{array}$ & $\begin{array}{c}\text { HIF- } 2 \alpha>\text { HIF- } 1 \alpha[3] \\
\text { C-ODD only [4] }\end{array}$ \\
\hline hypoxia-inducibility & no & yes & yes \\
\hline $\begin{array}{c}\text { chromosomal localization } \\
\text { (in mice) }\end{array}$ & 7 & 8 & 12 \\
\hline protein size [kDa] & 44 & 46 & 27 \\
\hline$K \mathrm{~m}$ values for $\mathrm{O}_{2}[\mu \mathrm{M}]$ & 230 & 250 & 230 \\
\hline
\end{tabular}

[1] Sharp and Bernaudin, 2004 [2] Lieb et al., 2002 [3] Appellhoff et al., 2004 [4] Hirsilä et al., 2003 [5] Metzen et al., 2003 


\section{Introduction}

\subsection{Mouse models}

\subsubsection{PHD knock-out mice}

To gain insight into the in vivo functions of the PHDs several PHD knock-out mouse models have been established.

Takeda et al. (2006) created constitutive PHD1 (targeted Exon: 3), PHD2 (targeted Exon: 2) and PHD3 (targeted Exon: 2) knock-out mice (PHD1 $\left.{ }^{-/}, \mathrm{PHD}^{-{ }^{-}}, \mathrm{PHD}^{-/}\right)$. While $\mathrm{PHD}^{-/-}$and $\mathrm{PHD}^{-/-}$mice seem apparently normal, $\mathrm{PHD}^{-/-}$mice die between E12.5 and E14.5. These embryos display severe symptoms like retardation in heart development, an open interventricular septum, thinner compact layer of ventricles, enlarged atria (which were congested with fetal erythrocytes), a malformed placenta and a pale yolk sac.

To further study the function of PHD2 in vivo, two groups created two independent conditional PHD2 knock-out mouse models (Minamishima et al., 2007; Takeda et al., 2007). The tamoxifen-inducible PHD2 knock-out mouse model created by Minamishima et al. (targeted Exons: 2 and 3) is restricted viable as these mice die suddenly at the age of about ten weeks. The knock-out mice are significantly smaller than their wt littermates and exhibit profound increases in red blood cell production which goes along with increased serum erythropoietin levels. Histological examination reveals venous congestion and occasional retroperitoneal hemorrhage. It has also been shown that these mice develop a dilated cardiomyopathy with enlarged cardiomoycytes. Furthermore signs of early cardiac ischemia were shown by an increased contraction band necrosis. But it was not analyzed whether these symptoms are an indirect consequence of polycythemia and hyperviscosity or if it is a direct consequence of chronic high-level HIF activation in cardiomyocytes.

The PHD2 knock-out mouse model created by Takeda et al. (targeted Exon: 2) is viable. These mice showed a hyperactive angiogenesis and angiectasia in multiple organs, including lung, liver and the heart.

\subsubsection{Transgenic HIF-1 $\alpha$ mice}

To study the function of HIF, which is normally only active in a hypoxic environment, in normoxia, HIF-1 $\alpha$ transgenic mice were created. Bekeredjian et al. (2010) created a transgenic mouse model carrying the human HIF-1 $\alpha$ CDNA under the control of the $\alpha M H C$ promoter with alanine substitutions at Pro ${ }^{402}$, Pro ${ }^{564}$ and $\mathrm{Asn}^{803}$ which makes HIF-1 $\alpha$ oxygeninsensitive. A doxycycline-inducible construct (tet-off system) was used to obtain tight 


\section{Introduction}

exogenous regulation of transgene expression. Echocardiographic analysis showed a marked dilation of the left ventricle and a decrease in fractional shortening but only modest signs of cardiac hypertrophy after doxycycline withdrawal. After restoring doxycycline, the heart function returned to normal. Despite the worsened heart function the myocardium showed no obvious abnormalities. No increased apoptosis, no fibrosis, no hypertrophy, nor heterogeneity in myocyte size could be detected. Cardiac transcript array analysis revealed an up-regulation of 213 genes and a down-regulation of 100 genes three days after doxycycline removal. The most notably regulated genes fulfill functions in metabolism, especially in glycolysis. In addition, genes regarding the calcium handling of the cell were regulated. The sarcoplasmic/endoplasmic reticulum calcium ATPase (SERCA), phospholamban (PLB) and the type-2 ryanodine receptor (RyR2) were also reduced on mRNA level with an impaired $\mathrm{Ca}^{2+}$ uptake after one week of HIF transgene expression.

Kido et al. (2005) investigated whether constitutive expression of HIF-1 $\alpha$ influences infarct size and cardiac performance after myocardial infarction. To answer this question a transgenic mouse was created carrying the unmodified HIF-1 $\alpha$ coding sequence under the control of the $\alpha \mathrm{MHC}$ promoter which restricts the expression of the transgene primarily to cardiomyocytes.

Transgenic (HIF-1 $\alpha(+))$ and age matched wt mice (HIF-1 $\alpha(-))$ underwent ligation of the left anterior descending artery (LAD) and were analyzed 24 hours and four weeks after ligation. 24 hours after the ligation HIF-1 $\alpha(+)$ and HIF-1 $\alpha(-)$ had comparable infarct sizes and areas at risk as determined by Evans Blue/ triphenyltetrazolium chloride (TTC) staining. However, it was shown that HIF-1 $\alpha(+)$ mice had a smaller infarct size than HIF-1 $\alpha(-)$ with a bigger viable left ventricle area four weeks after the ligation. The cardiac function was likewise better preserved in HIF-1 $\alpha(+)$ mice as indicated by a significantly higher fractional shortening and ejection fraction. A contributing factor could be the increased angiogenesis that could be detected in the infarct and the peri-infarct region four weeks after ligation. This mouse strain was further analyzed in this thesis. 


\section{Introduction}

\subsection{Cardiovascular diseases}

In this thesis several cardiovascular diseases are mimicked and analyzed in the different mouse models. These are cardiomyopathies, myocardial infarction and an elevated mechanical load.

\subsubsection{Cardiomyopathies}

Cardiomyopathies, or generally spoken heart muscle diseases are diseases, in which the heart is abnormally enlarged, thickened and/or stiffened. There are several different types of cardiomyopathies. Some important ones are:

-Dilated cardiomyopathy (DCM) which is the most often occuring form. It is a condition in which the heart becomes weakened and enlarged so that the blood cannot be pumped efficiently anymore. The decreased heart function can affect the lung, liver, and other organs (Maron et al., 2006).

-Ischemic cardiomyopathy (ICM) is defined as a state of the heart in which it cannot pump enough blood to supply the whole body. This is due to coronary artery disease, a buildup of a hard substance (plaque) in the coronary arteries (Maron et al., 2006).

-Hypertrophic cardiomyopathy (HCM) is a condition in which the heart muscle thickens. This thickening can appear asymmetrical so that one part of the heart is thicker than others. Through the hardening it is more difficult for the blood to leave the heart which forces the heart to pump even harder. HCM is caused by a variety of mutations encoding contractile proteins of the cardiac sarcomere (Maron et al., 2006).

\subsubsection{Mechanical load: preload versus afterload}

Load is a critical factor of myocardial function. The two types of load are preload and afterload. Preload builds up during diastolic filling and stretches the cardiomyocytes. Afterload is the tension or stress developed in the wall of the left ventricle during ejection. In a mouse model an elevated afterload is achieved through transverse aortic constriction (TAC) (Toischer et al., 2010). In this surgery a ligation with a defined diameter is placed around the aorta transversa (Laufs et al., 2008). This surgery mimics another important heart diseasean aortic valve stenosis in which the opening of the aortic valve is narrowed. This narrowing hinders the blood from easily flowing into the aorta and then into the body. The heart can initially compensate this by thickening of its walls which leads to cardiac hypertrophy. But in 


\section{Introduction}

later stages of the disease progress the left ventricle dilates, the wall becomes thinner and in addition the systolic function decreases.

\subsubsection{Myocardial Infarction}

Myocardial infarction is the irreversible necrosis of the heart muscle secondary to prolonged ischemia. This usually results from an imbalance between oxygen supply and demand, which is most often caused by blood clots in a coronary vessel, resulting in an acute reduction of blood and oxygen supply to a portion of the myocardium.

To mimic this disease pattern in a mouse model the left anterior descending artery can be surgically ligated (Patten et al., 1998). Afterwards the area at risk and the area of necrosis can be determined by Evan's Blue/TTC staining (Bohl et al., 2009). Furthermore, the cardiac remodeling can be analyzed by echocardiography over time. 


\section{Original Publications}

\section{Original Publications}

This chapter contains the following original articles which are published at the submission date of this thesis:

3.1. Marion Hölscher, Monique Silter, Sabine Krull, Melanie von Ahlen, Amke Hesse, Peter Schwartz, Ben Wielockx, Georg Breier, Dörthe M. Katschinski, Anke Zieseniss (2011)

Cardiomyocyte-specific Prolyl-4-hydroxylase Domain 2 Knock Out Protects from Acute Myocardial Ischaemic Injury

J Biol Chem 286:11185-11194

3.2. Marion Hölscher, Katrin Schäfer, Sabine Krull, Katja Farhat, Amke Hesse, Monique Silter, Yun Lin, Bernd Pichler, Patricia Thistlethwaite, Ali El-Armouche, Lars S. Maier, Dörthe M. Katschinski, Anke Zieseniss (2012)

Unfavourable consequences of chronic cardiac HIF-1 $\alpha$ stabilization

Cardiovasc Res 94:77-86 


\section{Original Publications}

\subsection{Cardiomyocyte-specific Prolyl-4-hydroxylase Domain 2 Knock Out Protects from Acute Myocardial Ischemic Injury}

The proly-4-hydroxlase domain enzyme 2 (PHD2) is the most important isoform of the PHDs based on its ubiquitous expression pattern and its dominant effect on HIF-1 $\alpha$ in normoxia. PHD2 knock-out embryos die between E12.5 and E14.5 which coincidences with increased levels of PHD2 in wt animals (Takeda et al., 2006). To further analyze the consequences of PHD2 loss in vivo two independent PHD2 knock-out mouse models have been created (Minamishima et al., 2007; Takeda et al., 2007). The phenotype of these mice is similar to the consequences of HIF-1 $\alpha$ overexpression with increased angiogenesis, erythropoiesis and extramedullar hematopoiesis. These mice also show signs of a cardiac phenotype with symptoms of a dilated cardiomyopathy. But as these mice also have an increased hematocrit, an indirect effect via the increased blood viscosity cannot be excluded.

To gain deeper insight into the direct functions of PHD2 in the heart, we created a cardiomyocyte-specific PHD2 knock-out mouse model (cPhd $\left.{ }^{--}\right)$and analyzed these mice in comparison to $w t$ littermates.

In the following publication the successful generation of these cardiomyocyte-specific PHD2 knock-out mice is described. The mice were analyzed with regard to HIF-1 $\alpha$ stabilization, heart function, capillaries and ultrastructure in the heart. Furthermore, these mice underwent transverse aortic constriction and permanent ligation of the left anterior descending artery.

In summary, I was able to show that these mice show elevated HIF-1 $\alpha$ protein levels with subsequent up-regulation of HIF target genes. $C P h d 2^{--}$mice do not respond differently to an increased mechanical load by transverse aortic constriction compared to their wt littermates. After ligation of the left anterior descending artery, however, the area at risk and area of necrosis were significantly smaller in $c P h d 2^{-/-}$mice compared with Phd2 wt mice. This is most likely due to an increased capillary area as a consequence of pro-vasodilatory pathways induced by HIF. 


\title{
Cardiomyocyte-specific Prolyl-4-hydroxylase Domain 2 Knock Out Protects from Acute Myocardial Ischemic Injury*[s
}

Received for publication, September 20,2010, and in revised form. January 15. 2011 Published. IEC Papers in Press. January 26. 2011, DOI 10.1074/Jbc M1 10.186809

\author{
Marion Hölscher ${ }^{\ddagger}$, Monique Silter ${ }^{\ddagger}$, Sabine Krull ${ }^{\ddagger}$, Melanie von Ahlen $^{\ddagger}$, Amke Hesse $^{\ddagger}$, Peter Schwartz \\ Ben Wielockx", Georg Breier", Dörthe M. Katschinski ${ }^{\ddagger 1}$, and Anke Zieseniss ${ }^{\ddagger}$ \\ From the ${ }^{\ddagger}$ Department of Cardiovascular Physiology, Universitätsmedizin Göttingen, Georg-August University Göttingen, \\ D-37073 Göttingen, Germany, the ${ }^{5}$ Department of Anatomy and Embryology, Universitätsmedizin Göttingen, Georg August \\ University Göttingen, D-37075 Göttingen, Germany, and the "Department of Pathology, TU Dresden, D-01307 Dresden, Germany
}

Prolylhydroxylase domain proteins (PHD) are cellular oxygen-sensing molecules that regulate the stability of the $\alpha$-subunit of the transcription factor hypoxia inducible factor (HIF)- 1 . HIF-1 affects cardiac development as well as adaptation of the heart toward increased pressure overload or myocardial infarction. We have disrupted PHD2 in cardiomyocytes (cPhd ${ }^{-1-}$ ) using $P h d 2^{\text {fox/fox }}$ mice in combination with MLCvCre mice, which resulted in $\mathrm{HIF}-1 \alpha$ stabilization and activation of HIF target genes in the heart. Although $\mathrm{CPhd} 2^{-t-}$ mice showed no gross abnormalities in cardiac filament structure or function, we observed a significant increased cardiac capillary area in those mice. $c P h d 2^{-t-}$ mice did not respond differently to increased mechanical load by transverse aortic constriction compared with their wild-type (wt) littermates. After ligation of the left anterior descending artery, however, the area at risk and area of necrosis were significantly smaller in the $c P_{h d} 2^{-t-}$ mice compared with $P h d 2 w t$ mice in line with the described pivotal role of HIF- $1 \alpha$ for tissue protection in case of myocardial infarction. This correlated with a decreased number of apoptotic cells in the infarcted myocardium in the $\mathrm{CPhd2^{-/- }}$ mice and significantly improved cardiac function 3 weeks after myocardial infarction.

When oxygen availability is impaired, the resulting hypoxia activates homeostatic mechanisms at the systemic and cellular level(1). Hypoxia-inducible factors (HIFs) ${ }^{2}$ are essential players in these responses because they regulate the transcription of a

* This work was supported by Kröner Fresenius Stiftung Grant P25/10// A12/10 (to A.Z) and Deutsche Forschungsgemeinschaft Grants Ka1269/ $11-1$ (to D. M. K), Bt 1336/2-3 and SFB 655 A 8 (to G. B.), and Wi 3291/1-1 (to B. W.). The work was performed as collaborative project within the COST Action TD0901 "HypoxiaNet."

5. The on-line version of this article (available at http://mww.jbc.org) contains supplemental Figs. 1 and 2.

'To whom correspondence should be addressed: Dept. of Cardiovascular Physiology, Georg-August University Göttingen, Humboldtallee 23 D-37073 Göttingen, Germany. Tel: 49551395648 ; Fax: 49551395895 ; E-mail katschinskiephysiol,med.uni-goettingen,de.

${ }^{2}$ The abbreviations used are: HIF, hypoxia-inducible factor; AAR, area at risk; Ang, angiopoietin; AON, area of necrosis; Bnip3, BCL2/adenovirus E1B $19-\mathrm{kDa}$ protein-interacting protein 3 ; BNP, brain natriuretic peptide; $c$ Phd2 $2^{-/-}$, cardiac-specific PHD2 knockout; E, embryonic day: FS, fractional shortening; Glut-1, glucose transporter-1; Hox-1, hemoxygenase; iNOS inducible nitric-oxide synthase: $\mathrm{LAD}$, left anterior descending artery: $\mathrm{PDK} 1$, pyruvate dehydrogenase kinase 1; pfk1, phosphofruktokinase 1; PGK phosphoglycerate kinase; PHD, prolylhydroxylase domain; TAC, transverse aortic constriction; $\Pi C, 2,3,5$-triphenyltetrazolium chloride; $w$, wild type. large number of genes that affect a myriad of cellular processes, including angiogenesis, metabolism, cell survival, and oxygen delivery (2). HIF is a heterodimeric protein comprising the oxy. gen-sensitive $\alpha$-subunit HIF- $1 \alpha$ or the more cell type-specifically expressed HIF- $2 \alpha$ or HIF- $3 \alpha$ and the oxygen-insensitive $\beta$-subunit (3). In the presence of oxygen, HIF $\alpha$ becomes hydroxylated at two critical proline residues by prolylhydroxy. lase domain (PHD) enzymes $(4,5)$. The PHD protein family responsible for $\mathrm{HIF} \alpha$ regulation consists of three members called prolylhydroxylase domain (PHD)1, PHD2, and PHD3 (6, 7). Following prolyl-4-hydroxylation of the critical prolyl residues under normoxic conditions, the ubiquitin ligase von Hippel-Lindau tumor suppressor protein recognizes HIF- $1 \alpha$ subunits and targets them for rapid ubiquitination and proteasomal degradation $(8-10)$.

Based on the ubiquitous expression pattern and its dominant effect in normoxia, it had to be assumed that PHD2 is the most critical HIF-1 $\alpha$-regulating PHD isoform in most tissues (1113). This notion, learned from in vitro studies, was confirmed by the up to now available genetically modified $P h d 2$ mouse models (14). Phd 2 knock-out embryos die between embryonic day (E) 12.5 and E14.5 (15). This time point coincides with the increased levels of PHD2 in wild-type (wt) mice starting from E9.0. A major role of PHD2 in regulating the HIF system is further underscored by mouse models with a somatic Phd $2^{-{ }^{\prime-}}$ knock out, which enable to analyze the in vivo function of PHD2 in the adult mice. Two independent inducible $\mathrm{Phd} 2^{-t-}$ mouse models were developed by Takeda et al. (16) and Minamishima et al. (17). The phenotype of these mice most obviously resembles the consequences of HIF $\alpha$ overexpression with increased angiogenesis, erythropoiesis, and extramedullar hematopoiesis $(17,18)$. Most interestingly, these mice also develop a cardiac phenotype with symptoms of dilated cardiomyopathy. In the heart, HIF- $1 \alpha$ and thereby also the PHDs are known to influence key components of heart development, morphogenesis, and function $(19,20)$. Long term activation of HIF- $1 \alpha$ in the heart seems to activate detrimental pathways resulting in the development of heart failure (21). Thus, it is tempting to speculate that loss of PHD2 in the heart is responsible for the dilated cardiomyopathy as observed in the inducible $P h d 2^{-1-}$ mice. However, because these mice also develop an increased hematocrit, secondary blood hyperviscosity-dependent cardiac changes cannot be ruled out.

Therefore we have generated cardiomyocyte-specific PHD2 knock-out mice ( $c P h d 2^{-1-}$ ) by crossing $\mathrm{MLCvCre}$ mice with 


\section{Original Publications}

\section{PHD2 and Myocardial Ischemia}

Phd $2^{\text {floxfflox }}$ mice. While this work was ongoing Moslehi et al. have recently reported the generation of cardiac-specific PHD2 knock-out mice using $\alpha M H C C r e$ expressing mice as deleter mice in combination with $P h d 2^{\text {flox flox }}$ mice (22). In line with our observation, Moslehi et al. observed no striking differences in morphology or function of resting young $c P h d 2^{-1-}$ mice. Moslehi et al. additionally analyzed older mice and demonstrated that with increasing age cardiomyocyte-specific PHD2 knock-out mice developed changes, which phenocopy ischemic cardiomyopathy. At a young age these animals responded to increased afterload with fortified heart failure despite the successful development of cardiac hypertrophy.

In contrast to the results of Moslehi et al., we did not observe significant changes in the development of hypertrophy and heart failure of young PHD2-deficient mice toward increased afterload in our animal model. Moreover, we observed cardioprotective effects in the PHD2-lacking mice in a model of myocardial ischemia. Challenging the mice with permanent ligation of the left anterior descending artery (LAD) resulted in cardiac tissue protection and decreased myocardial infarct size in the cPhd $2^{-t-}$ mice compared with Phd2 wt littermates. Taken together, our results show for the first time that lack of PHD2 in cardiomyocytes protects the heart from an acute ischemic insult. This phenomenon is at least in part most likely due to an increased capillary area in the $c P h d 2^{-1-}$ mice.

\section{EXPERIMENTAL PROCEDURES}

Mice-The generation and detailed characterization of Phd $2^{\text {flox flox }}$ mice will be reported elsewhere. ${ }^{3}$ In brief, LoxP sites were cloned into the $P h d 2$ gene via Red/ET recombination (23) flanking exons 2 and 3 . To characterize the $P h d 2^{\text {flox } f l o x}$ mice, full inactivation of the $P h d 2$ gene was achieved via an intercross with $P G K C r e$ mice. Consistent with earlier reports, no viable $P H D 2^{-1-}$ mice were obtained $(15,17)$.

Cre-mediated excision of exons 2 and 3 results in a frameshift mutation from exon 1 to 4 leading to an early translational stop and subsequent Phd 2 knock out. Exons 2 and 3 encode for almost the entire catalytic domain of Phd2. The successful deletion of Phd 2 in the floxed mice was verified by Western blotting on protein extracts of embryonic hearts (E14.5) from PGKCre $\times$ Phd $2^{\text {flox flox }}$ mice using a homemade Phd 2 antibody against a C-terminal peptide (supplemental Fig. 1). No PHD2 protein could be detected in $P h d 2^{-1-}$ hearts. Moreover, the fact that HIF- $1 \alpha$ protein is stabilized in these samples verifies the loss of functionality of Phd2. To analyze, if a shorter PHD2 protein encoded by exon 1 is formed, we performed Western blotting using an antibody, which detects the $\mathrm{N}$-terminal part of PHD2 (supplemental Fig. 2). There is indeed a shorter Phd2 protein detectable in the $P h d 2^{+/-}$and $P h d 2^{-1-}$ mice, which in its apparent molecular mass matches the predicted number of $31.5 \mathrm{kDa}$. The putatively exon 1-encoded protein, however, was expressed compared with the wt PHD2 protein in wt or $P h d 2^{+1-}$ embryos only at very low levels. Whether this shorter protein would have a dominant negative character and therefore would bind to its interaction partner (e.g. HIF $\alpha$ ) and block

${ }^{3}$ B. Wielockx, K. Anastassiadis, A. F. Stewart, and G. Breier, unpublished observations. its normal function has not been studied. However, it was suggested that in human PHD2 both $\mathrm{Arg}^{371}$ and $\mathrm{Pro}^{317}$ (in mice $\mathrm{Arg}^{348}$ and Pro ${ }^{294}$ located in exons 3 and 2, respectively) contribute to the HIF- $1 \alpha$ or HIF- $2 \alpha$ binding (24). In our mice these sites are not present anymore, and a dominant negative interaction with HIF- $1 \alpha$ is therefore unlikely.

All animals in this study were backcrossed to C57BL/6 mice at least five times. Phd $2^{\text {flox/flox }}$ mice were crossed with $\mathrm{MLCvCr} e^{+/-}$mice (25) to generate Phd $2^{\text {flox flox }} \times \mathrm{MLCvCre^{+/- }}$ mice within two generations. Phd flox/flox $\times M L C v C r e^{+1-}$ mice were then crossed with $P h d 2^{\text {flox/flox }}$ mice to obtain $c P h d 2^{-1-}\left(P h d 2^{\text {flox } / \text { flox }} \times\right.$ $\mathrm{MLCvCr} \mathrm{C}^{+/-}$) mice and littermate control wild-type mice $\left(P h d 2^{\text {flox/flox }}\right)$.

Mice were genotyped by PCR using the following primers: Cre forward, 5'-CGTACTGACGGTGGGAGAAT-3' and Cre reverse, 5'-CGGCAAAACAGGTAGTTA-3'; PHD2 forward, $5^{\prime}$-CTCACTGACCTACGCCGTGT-3' and PHD2 reverse, 5'-CGCATCTTCCATCTCCATTT-3'.

The deletion of floxed exons in cDNAs isolated from ventricles or atria by PCRs was shown with the following primers: PHD2 forward, 5' -TACAGGATAAACGGCCGAAC-3' and PHD2 reverse, 5'-GGCAACTGAGAGGCTGTAGG-3'. The forward primer binds in exon 1 , the reverse primer binds in exon 5.

Animal Experimentation and Echocardiography-Animal experimentation was performed with $c P h d 2^{-1-}$ mice and littermate $P h d 2 w t$ control mice. All protocols regarding animal experimentation were conducted according to the German animal protection laws and approved by the responsible governmental authority (Niedersächsisches Landesamt für Verbraucherschutz und Lebensmittelsicherheit in Oldenburg; animal experimentation numbers 33.942502-04-10/0024 and 33.9-42502-04-10/0069). Pressure overload was induced by transverse aortic constriction (TAC) and performed essentially as described previously by our group with blunted 27-gauge needles as placeholders in 10-week-old female mice (26).

LAD ligations were performed by an investigator who was blinded regarding the genotypes of the mice. The measurement of infarct size was performed by an additional investigator, who was likewise blinded.

LAD ligation was performed on female mice having a minimum weight of $21 \mathrm{~g}$ ( $8-12$ weeks of age). Mice were anesthetized ( $2 \%$ isoflurane). The trachea was surgically exposed, and tracheal intubation was performed. A blunt intubation cannula (intubation cannula, stainless steel with Y-adapter, $1.2-\mathrm{mm}$ outer diameter, 30-mm length; Hugo Sachs Elektronik, Harvard Apparatus $\mathrm{GmbH}$ ) was inserted into the trachea. Correct tube placement was confirmed by direct visualization of the cannula within the previously exposed trachea. The tracheal tube was connected to a mechanical ventilator (MiniVent; Hugo Sachs Elektronik, Harvard Apparatus GmbH), and the animals were ventilated by using a pressure-controlled ventilation mode (stroke volume $150 \mu \mathrm{l}, 150$ strokes/min fractional inspired $\mathrm{O}_{2}=0.3$ ). After exposing the heart, the pericardium was removed, and a 9-0 polyamide suture with a U-shaped needle was passed under the left anterior descending artery. The 


\section{Original Publications}

\section{PHD2 and Myocardial Ischemia}

suture was tied to occlude the artery. The chest was closed and the mouse recovered.

Echocardiography and measurement of posterior wall thickness, septum thickness, left ventricular end systolic diameter, left ventricular end diastolic diameter, and fractional shortening (FS) were performed as described by Silter et al. (26).

Measurement of Infarct Size-Six hours after LAD ligation the mice were given heparin ( 250 units) and anesthetized, and the hearts were excised. Myocardial infarct size was determined by using Evans blue/2,3,5-triphenyltetrazolium chloride (TTC) staining as described by Bohl et al. (27). Briefly, the ascending aorta was cannulated with a 20-gauge tubing adapter, and $1 \%$ Evans blue was perfused into the aorta and coronary arteries to delineate the "area at risk" (AAR). The Evans blue dye was distributed uniformly to those areas of the myocardium, which were well perfused; hence, the area of the myocardium that was not stained with Evans blue was defined as the AAR. The left ventricle was separated from the rest of the heart and sectioned into three transverse slices. Sections were incubated in $2 \%$ TTC for $20 \mathrm{~min}$ at $37^{\circ} \mathrm{C}$ to identify viable tissue. Infarct quantification was performed on digital photographs (SMZ 100; Nikon, Tokyo, Japan) using Imagel (National lnstitutes of Health). The area of necrosis $(A O N)$ and $A A R$ were determined as the average percent area per slice from the middle section and the lowest section. Also, myocardial samples were collected for cyrosectioning and assessment of apoptosis.

Protein Extraction and Immunoblot Analyses-Heart tissue was rapidly homogenized in a buffer containing $4 \mathrm{M}$ urea, 140 mM Tris $(\mathrm{pH} 6.8), 1 \%$ SDS, $2 \%$ Nonidet P-40, and protease inhibitors (Roche Applied Science). Protein concentrations were quantified (DC Protein Assay; Bio-Rad). For immunoblot analysis protein samples were resolved by SDS-PAGE and transferred onto nitrocellulose membranes (Amersham Biosciences) by semidry blotting (PeqLab). Primary antibodies used were: anti-PHD2 (NB100-2219; Novus), anti-HIF-1 $\alpha$ (NB100479; Novus), and anti-vinculin (hVin-1, V9264; Sigma).

For detection of immunocomplexes, horseradish peroxidase-conjugated secondary goat anti-rabbit or goat anti-mouse antibodies (Santa Cruz Biotechnology) were used, and membranes were incubated with $100 \mathrm{mM}$ Tris- $\mathrm{HCl}$ (p H 8.5), $2.65 \mathrm{~mm}$ $\mathrm{H}_{2} \mathrm{O}_{2}, 0.45 \mathrm{~mm}$ luminol, and $0.625 \mathrm{~mm}$ coumaric acid for $1 \mathrm{~min}$. Chemiluminescence signals were detected with the LAS3000 camera system (Fuji Film Europe, Düsseldorf, Germany).

RT-PCR Analyses-After RNA extraction reverse transcription (RT) was performed with $2 \mu \mathrm{g}$ of RNA and a first strand cDNA synthesis kit (Fermentas, St. Leon-Rot). mRNA levels were quantified by using $1 \mu \mathrm{l}$ of the cDNA reaction and a SYBR Green qPCR reaction kit (Clontech) in combination with a MX3000P light cycler (Stratagene). The initial template concentration of each sample was calculated by comparison with serial dilutions of a calibrated standard. Primers were as follows: glucose transporter-1 (Glut-1) forward, 5' - TGGCCTTGCTGGAACGGCTG and Glut-1 reverse, 5 ' -TCCTTGGGCTGCAGGGAGCA-3'; mS12 forward, 5'-GAAGCTGCCAAGGCCTTAGA-3 and mS12 reverse, 5'-AACT GCAACCAACCACCTTC-3'; PHD2 forward, 5'-TTGCTGACATTGAACCCAAA-3' and PHD2 reverse, 5'-GGCAACTGAGAGGCTGTAGG-3'; PHD1 forward, 5'-GCTAGGCTGAGGGAGG-
AAGT-3' and PHD1 reverse, $5^{\prime}$-TCTACCCAGGCAATCTGGTC-3'; PHD3 forward, 5'-GGCCGCTGTATCACCTGT. AT-3 and PHD3 reverse, $5^{\prime}$ - TTCTGCCCTTTCTTCAGCAT$3^{\prime}$; brain natriuretic peptide (BNP) forward, 5 ' -AAGTCCTAGCCAGTCTCCAGA-3' and BNP reverse, 5'-GAGCTGTCTCTGGGCCATTTC-3'; phosphofruktokinase 1 (Pfkl) forward, $5^{\prime}$-ACGAGGCCATCCAGCTCCGT-3' and Pfkl reverse $5^{\prime}$ - TGGGGCTTGGGCAGTGTCCT-3'; pyruvate dehydrogenase kinase 1 (PDK-1) forward, 5'-GTTCACGTCACGCTGGGCGA-3' and PDK-1 reverse, 5' -CCAGGCGTCCCATGTGCGTT-3'; phosphoglycerate kinase (PGK) forward, 5 '-ACGTCTGCCGCGCTGTTCTC-3' and PGK reverse, 5' -ACCATGGAGCTATGGGCTCGGT-3'; VEGF forward, 5'-CGAACGTACTGCCGATTGAGA-3 and VEGF reverse, 5 ' - TGGTGAGGTTTGATCCGCATGATCTG-3'; angiopoietin (Ang)-1 forward, 5'-CGCTCTCATGCTAACAGGAGGTTGG-3' and Ang-1 reverse, 5'-GCATTCTCTGGACCCAAGTGGCG-3'; Ang-2 forward, 5'-CACCCAACTCCAAGAGCTCGG-3' and Ang-2 reverse, 5'-CACGTAGCGGTGCTGACCGG-3'; BCL2/adenovirus E1B 19-kDa protein-interacting protein 3 (Bnip3) forward, 5'-GTCCAGTGTCGCCTGGCCTC-3' and Bnip3 reverse, 5'-TGGGAGCGAGGTGGGCTGTC-3'; iNOS, inducible nitric-oxide synthase; (iNOS) forward, $5^{\prime}$ - GCTGCCTTCCTGCTGTCGCA-3' and iNOS reverse, 5'-GGAGCCGCTGCTGCCAGAAA-3; hemoxygenase (Hox)-1 forward, 5'. TGGTGCAAGATACTGCCCCTGC- $3^{\prime}$ and Hox-1 reverse, 5'-TGGGGGACAGCAGTCGTGGT-3', apelin forward, $5^{\prime}$-TGCAGTTTGTGGAGTGCCACTG-3' and apelin reverse, 5'-GCACCGGGAGGCACT-3'; adrenomedullin forward, 5'-TGGCCCCCTACAAGCCAGCAAT-3' and adrenomedullin reverse, 5'-GCCAACGGGATACTCGCCCG- 3 ' The sets of primers for adenosine receptor $A 2 b$ and $C D 73$ were according to previously published sequences (28).

$B N P$ Enzyme Immunoassay-Plasma BNP levels were measured using a commercially available enzyme immunoassay kit (catalog number EK-011-23; Phoenix Pharmaceuticals) according to the manufacturer's instructions.

Histological Analyses-For Trichrome staining, heart tissue was fixed in $4 \%$ paraformaldehyde in phosphate-buffered saline (PBS) and embedded in paraffin before sectioning.

Cryosectioning and Immunofluorescence Labeling-Freshly isolated hearts were treated as described before (29). Briefly, hearts were sectioned $(5-10 \mu \mathrm{m})$ in a cryostat, mounted on glass slides, and dried for at least $30 \mathrm{~min}$. After being washed with PBS, the sections were briefly incubated with $1 \%$ Triton X-100 and then fixed with $4 \%$ formaldehyde and permeabilized with $0.2 \%$ Triton $\mathrm{X}-100$. Nonspecific binding of antibodies was blocked by incubation with $1 \%$ bovine serum albumin for $1 \mathrm{~h}$. The sections were then incubated with the polyclonal antiCD31 antibodies (DLA 310, diluted 1:20; Dianova) and antivinculin antibodies ( $\mathrm{hVin}$-1, diluted 1:100; Sigma) at room temperature for $1 \mathrm{~h}$, washed three times in PBS, and then incubated with anti-mouse TR (diluted 1:200; Santa Cruz Biotechnology) and anti-rat FITC (1:200; Santa Cruz Biotechnology). Samples were counterstained for DNA (Hoechst). Finally, samples were washed extensively in PBS, mounted in Mowiol, and examined by fluorescence microscopy (Axio Observer D1; Carl Zeiss, Göttingen, Germany). The capillary area was determined by 


\section{Original Publications}

\section{PHD2 and Myocardial Ischemia}

A
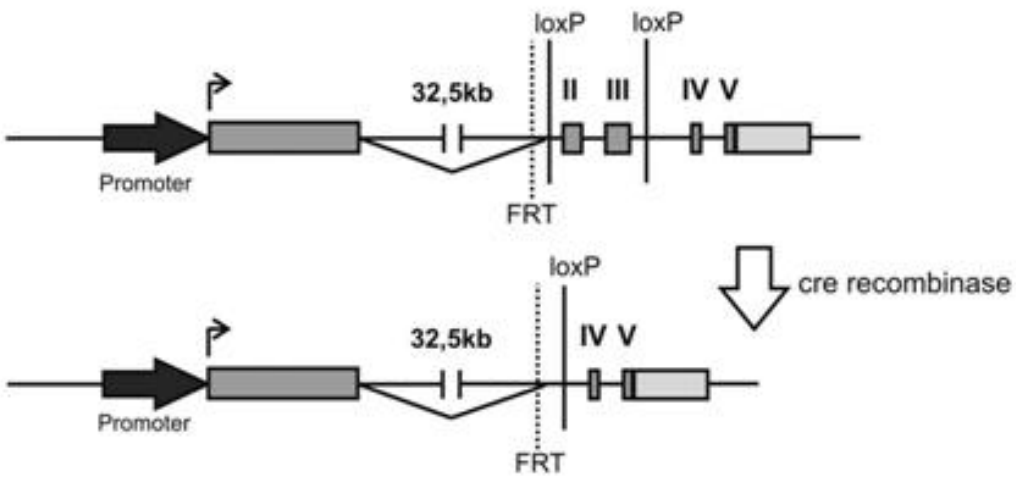

B
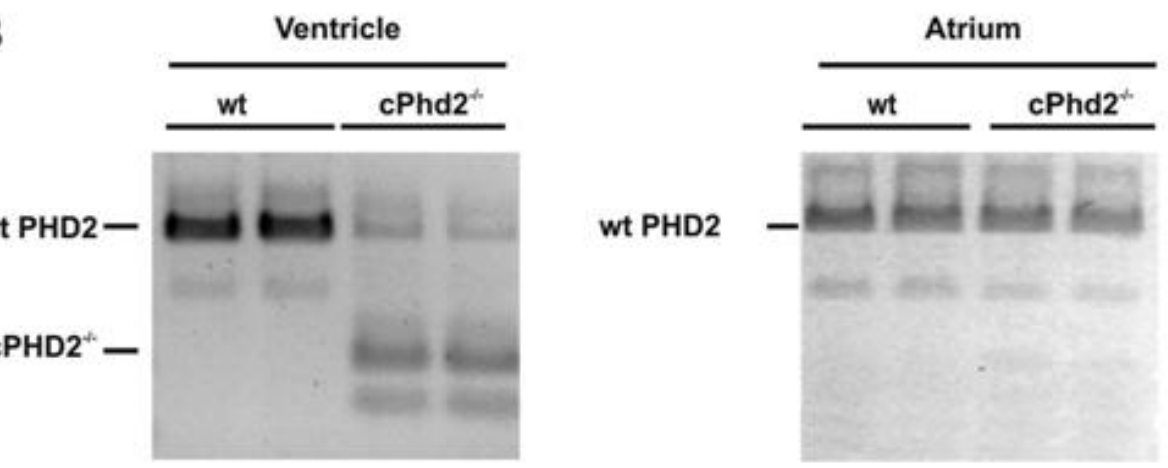

C

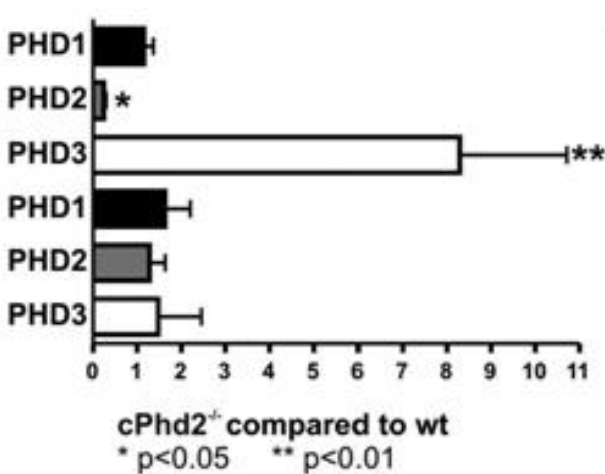

D

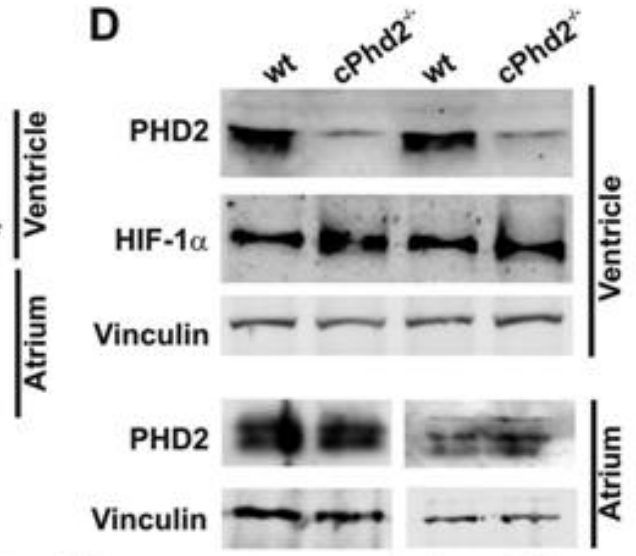

FIGURE 1. Generation and analyses of $c P h d 2^{-1-}$ mice. $A$, schematic description of the gene targeting strategy is shown. In the targeted Phd 2 locus, exons 2 and 3 are flanked by two loxP sites. Exons 2 and 3 are deleted by Cre-mediated recombination by crossing Phd ${ }^{\text {flox }}$ lox mice with MLCvCre mice. B. PCRs were performed with cDNAs isolated from left ventricles or atria of $c$ Phd2 $2^{-1-}$ or Phd2 wt control mice to demonstrate the deletion of floxed exons in the ventricles but not the atria. Incomplete recombination in the ventricles is most likely due to the presence of noncardiomyocytes in the tissue samples. Additional bands resemble splice variants. C. RT-PCR analysis confirmed the significant reduction of PHD2 mRNA transcripts in the left ventricles but not the atria of 8-week-old ${ }_{c}$ Phd $2^{-\gamma-}$ mice compared with wt littermates. Transcripts of the HIF-target PHD3 were increased whereas PHD2 mRNA was unchanged. In total, 11 wild-type mice and $7 \mathrm{CPHD}^{-\gamma-}$ were analyzed. ${ }^{*}, p<0.05 ; *, p<0.01$. Data represent mean values \pm S.E. (error bars). D, PHD2 protein was detected by Western blot analysis. Protein extracts prepared from left ventricles and atria of 8 -week-old Phd2 $w t$ and $c$ Phd $2^{-1-}$ mice were analyzed with anti-PHD2, anti-HIF-1 $\alpha$, and anti-vinculin antibodies, confirming that PHD2 was successfully knocked out in the left ventricles but not in the atria.

analyzing CD31-positive pixels using ImageJ. In addition, the number of capillaries were counted per view of field.

Immunohistochemistry-Paraffin-embedded sections were immunostained as described by others (22). The anti-HIF- $1 \alpha$ primary antibody (Novus NB100-123) was used at a 1:1000 dilution.

Electron Microscopy-Standard procedures were applied for fixation (1.5\% glutaraldehye and $1.5 \%$ paraformaldehyde in phosphate buffer) of hearts of $c P h d 2^{-1-}$ and Phd $2 w t$ mice. The hearts were then washed in PBS and fixed in $2 \%$ osmium tetroxide. The samples were dehydrated in a graded series of alcohol and embedded in Araldite. Ultrathin sections were stained with $2 \%$ methanolic uranyl acetate. Samples were then examined in a transmission electron microscope equipped with photodocumentation.

TUNEL Assay-Apoptotic cells were detected with a TUNEL. assay according to the manufacturer's protocol (In Situ Cell Death Detection kit, catalog number 11684795 001; Roche Applied Science). Three hearts of each genotype were analyzed, and five random high power fields from each heart sample were quantified.

Statistical Analyses-Data were analyzed by two-tailed Student's $t$ test or (in case of repeated echocardiography analysis after TAC treatment) as paired $t$ test and presented 


\section{Original Publications}

PHD2 and Myocardial Ischemia

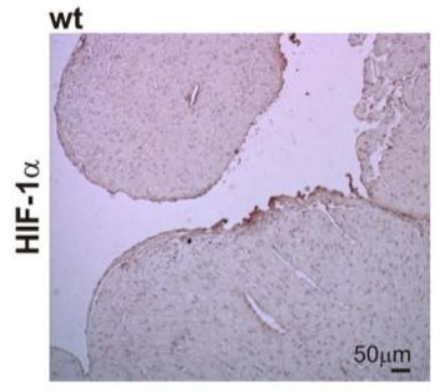

cPhd2
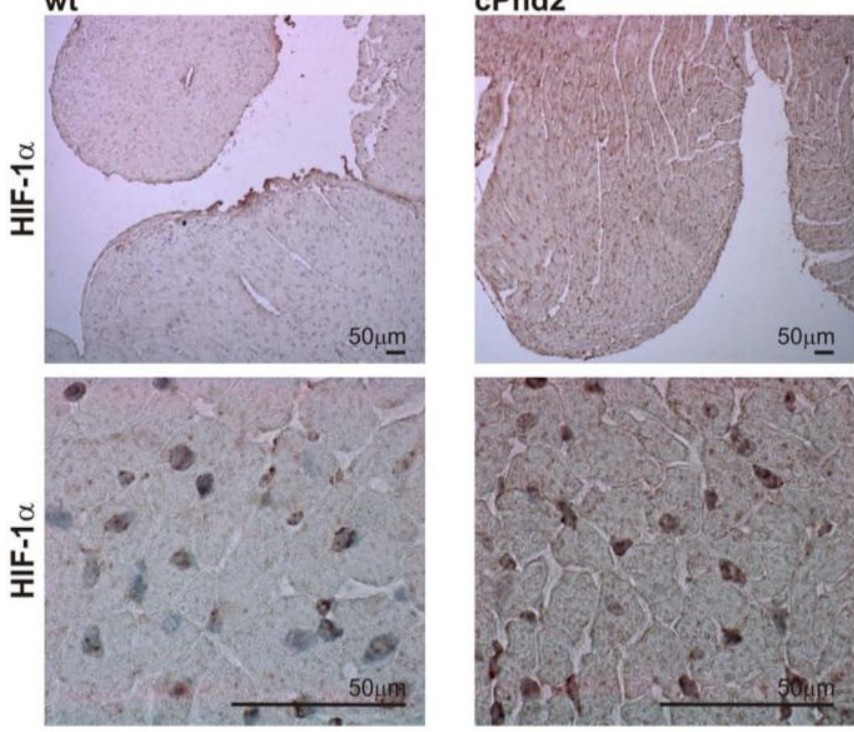

FIGURE 2. HIF-1 $\alpha$ protein accumulates in $\mathrm{cPhd2^{-1- }}$ hearts. Immunohistochemical analyses of hearts from 8-week-old $c P h d 2^{-1-}$ and $P$ hd 2 wt mice are shown. HIF- $1 \alpha$ protein accumulates in the nuclei of $c P h d 2^{-1-}$ ventricles.

as mean \pm S.E. A $p$ value $<0.05$ was considered statistically significant.

\section{RESULTS}

Generation of Cardiac-specific PHD2 Knock-out Mice-For generating $c P h d 2^{-1-}$ mice, we crossed Phd $2^{\text {flox/flox }}$ mice with mice that express the Cre recombinase under the control of the MLCv promoter (25). The MLCv-driven Cre permits recombination of the floxed Phd 2 exons 2 and 3 in ventricular cardiomyocytes (Fig. 1A). Isolation and PCR analysis of cDNAs from atria and ventricles of $c P h d 2^{-1-}$ and littermate Phd2 wt control mice verified successful recombination in the ventricles but not in the atria of the $c P h d 2^{-1-}$ mice (Fig. $1 B$ ). The additional bands seen in the PCR analysis of Phd $2 w t$ mice most likely are the result of described PHD2 splice variants (30).

Quantification of PHD2 RNA levels in the left ventricles and atria of $c P h d 2^{-1-}$ mice compared with $P h d 2 w t$ mice revealed a roughly 5 -fold reduction in the knock-out ventricles but not the atria, confirming the MLCvCre-mediated knock out in the ventricular cardiomyocytes (Fig. 1C). Considering that the RNA was isolated from the whole left ventricles, which besides the cardiomyocytes also contain especially cardiac fibroblasts but also endothelial cells, smooth muscle cells etc., this reduction in PHD2 expression is in accordance with a successful recombination in the Cre-expressing cardiomyocytes. In line, cardiac PHD2 expression was also reduced at the protein level in left ventricles but not the atria of $c P h d 2^{-1-}$ mice as determined by immunoblot analysis (Fig. 1D). As a consequence of the PHD2 knock out, HIF-1 $\alpha$ protein levels were slightly increased (Fig. $1 D)$. Immunohistochemical analysis confirmed the accumulation of HIF- $1 \alpha$ protein in cell nuclei of the cardiomyocytes (Fig. 2). Detection of HIF- $2 \alpha$ by immunohistochemistry was hampered by varying qualities of the batches of the commercially available polyclonal anti-HIF- $2 \alpha$ antibodies. At least in vitro it was described that in contrast to PHD3, PHD2 seems to have

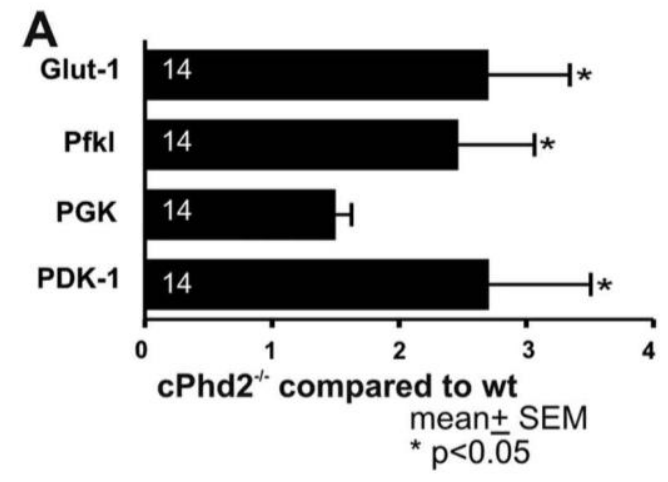

B
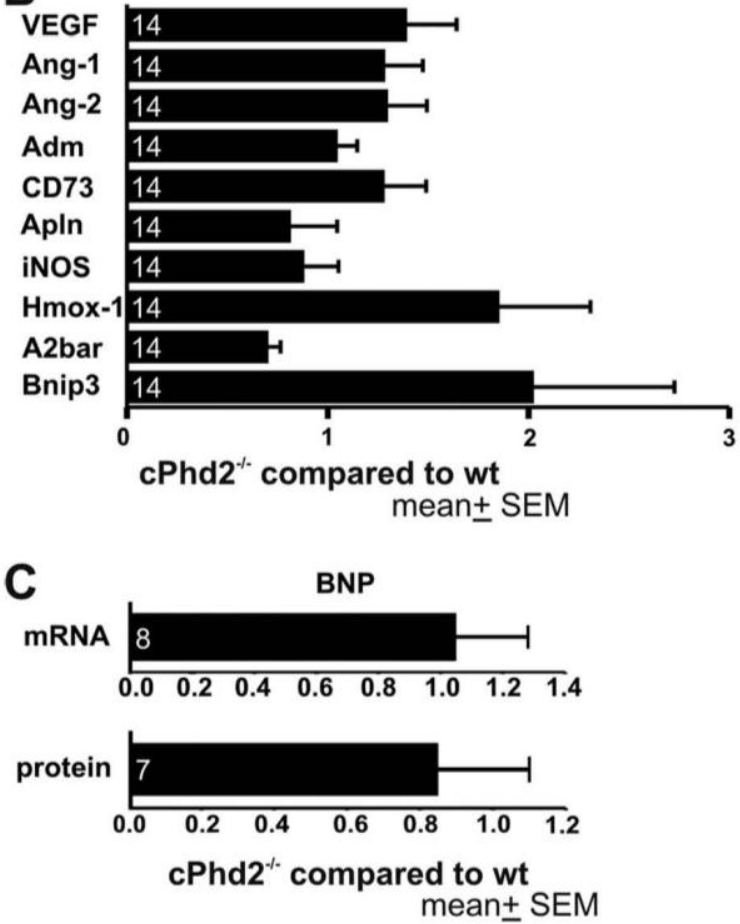

FIGURE 3. PHD2-deficient ventricles exhibit increased expression of HIF target genes. $A$ and $B$, quantitative real-time RT-PCR (qPCR) analysis of left ventricles from 8 -week-old $c P h d 2^{-1-}$ or Phd 2 wt mice was performed. Transcript levels of genes involved in glucose transport and glucose metabolism (A) as well as HIF-target genes related to angiogenesis, vasotonus regulation, and apoptosis $(B)$ were analyzed. The numbers in the bars indicate the number of animals analyzed. ${ }^{*}, p<0.05$. Data represent mean values \pm S.E. (errorbars). $C$, changes of BNP mRNA in the left ventricles or in serum protein levels of CPhd $2^{-1-}$ compared with Phd 2 wt mice were not detectable by qPCR and ELISA, respectively. Data represent mean values \pm S.E.

higher activity toward HIF- $1 \alpha$ compared with HIF- $2 \alpha$ (30). Therefore the increased levels of HIF- $1 \alpha$ in the cardiomyocytes are in line with an effective PHD2 knock out in our mouse model. A moderate activation of the HIF-signaling pathway in the hearts of $c P h d 2^{-1-}$ mice was likewise detected by analyzing the expression of the HIF target genes PHD3 (Fig. 1C), Glut-1, Pfk1, and PDK1 (Fig. 3A). Whereas increased levels of Glut-1, Pfk1, and PDK1 reflect the impact of HIF on glucose transport and glucose metabolism, respectively (31), the elevated levels of PHD3 are indicative of the described negative HIF/PHD feedback loop, which involves the HIF-dependent transcription of PHD3 (32). In contrast, PHD1 RNA levels, which are not induc- 


\section{Original Publications}

\section{PHD2 and Myocardial Ischemia}

A wt

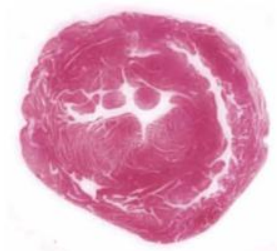

B
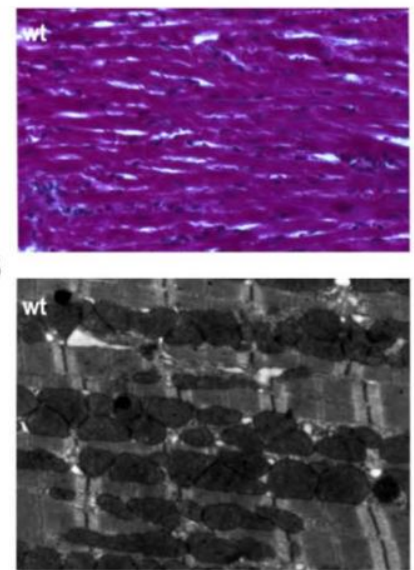

cPhd2
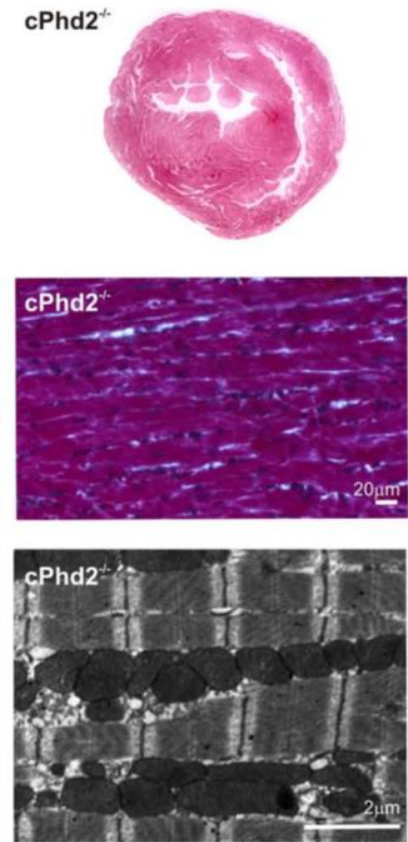

FIGURE4.CPhd2 ${ }^{-1-}$ hearts show no gross structural abnormalities. $A$, paraffin-embedded tissue sections of Phd2 wt and $c P h d 2^{-1-}$ hearts stained with Trichrome. B, transmission electron microscopy of the Phd2 wt and $C P h d 2^{-1-}$ hearts.
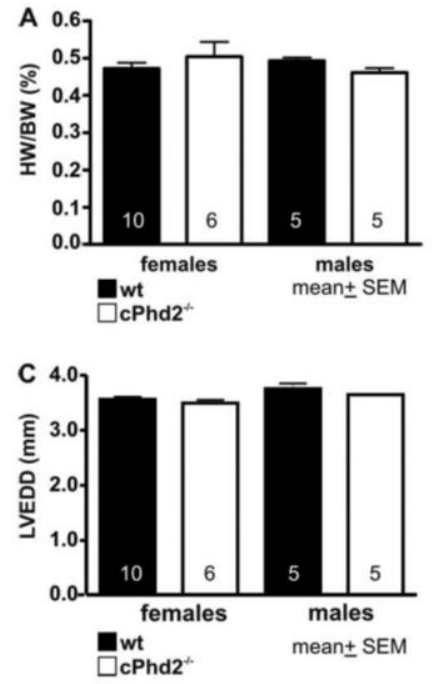
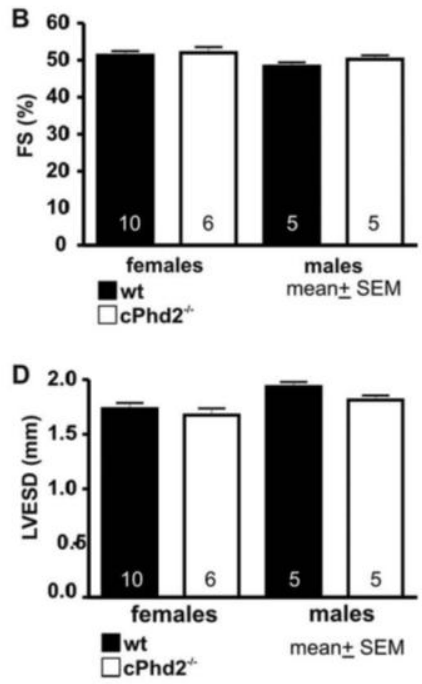

FIGURE 5. Cardiac PHD2 deficiency does not affect heart function in 8-week-old mice. $A$, hearts of $P h d 2$ wt or $C P h d 2^{-1-}$ mice were excised, and the ratios of heart weight ( $H W$ ) compared with body weight ( $B W$ ) were determined. Data represent mean values \pm S.E. (error bars). The numbers in the bars indicate the number of animals analyzed. $B-D$, fractional shortening $(F S)(B)$ left ventricular end diastolic diameter (LVEDD) $(C)$, and left ventricular end systolic diameter (LVESD) (D) were analyzed by echocardiography and showed no differences between indicated genotypes. The numbers in the bars indicate the number of animals analyzed. Data represent mean values \pm S.E.

ible by hypoxia (13), were not affected in the hearts of $c P h d 2^{-1-}$ mice (Fig. 1C). HIF target genes involved in angiogenesis (VEGF, Ang-1, Ang-2), vasotonus regulation (adrenomedullin, apelin, iNOS, and Hox-1), purinergic signaling (CD73, and adenosine receptor $\mathrm{A} 2 \mathrm{~b}$ ), and apoptosis (Bnip3) were found to be unchanged (Fig. $3 B$ ). BNP is produced in the ventricles and is regarded as surrogate marker for heart failure (33). Analyzing

$A_{\text {w }}$
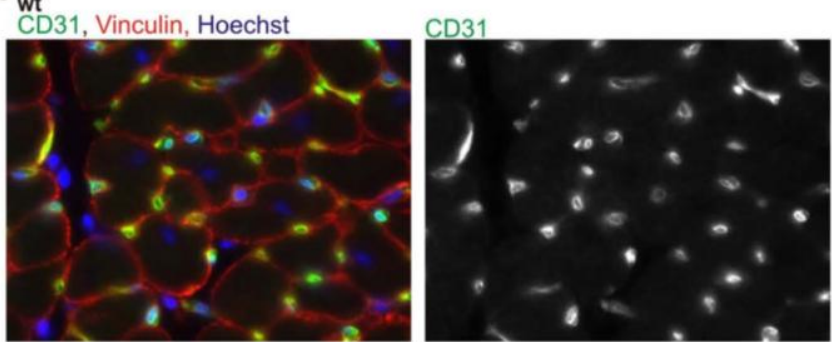

cPhd2 $^{\text {t- }}$
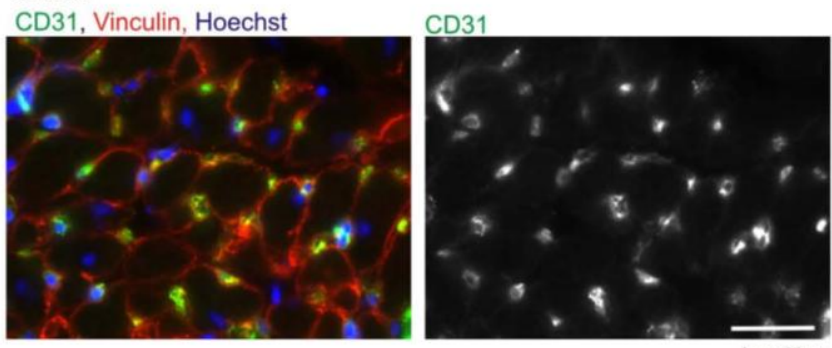

bar: $20 \mu \mathrm{m}$

\section{B}
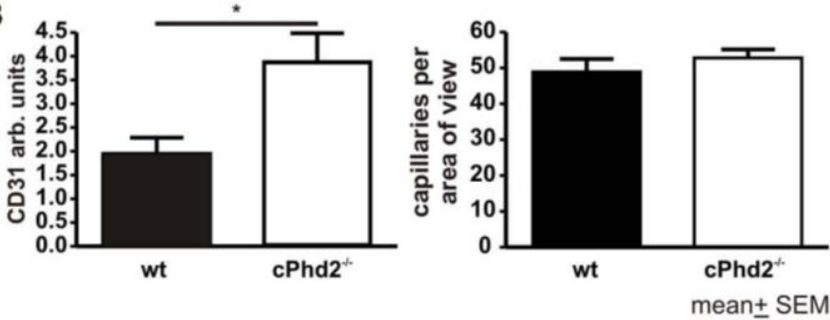

FIGURE 6. Increased capillary area in PHD2-deficient hearts. $A$, hearts of cPhd $2^{-1-}$ mice $(n=4)$ and Phd 2 wt $(n=4)$ littermates were excised and analyzed for angiogenesis as determined by anti-CD31 staining. Sections were co-stained for vinculin and DNA (Hoechst). B, the area of capillaries, which were determined in $A$, were quantified by determining the extent of CD31-positive staining, and the number of capillaries were counted. *, $p<$ 0.05 . Data represent mean values \pm S.E. (error bars).

BNP RNA levels in the left ventricles or BNP plasma levels of $P h d 2 w t$ and $c P h d 2^{-1-}$ mice revealed no significant differences, indicating that there were no obvious signs for heart failure in the $c P h d 2^{-1-}$ mice (Fig. 3C).

Histological assessment of the heart revealed no gross abnormalities in the $c P h d 2^{-1-}$ mice (Fig. $4 A$ ). Furthermore, electron microscopy analysis displayed no significant changes in ultrastructure or filament architecture (Fig. 4B). Heart weight (Fig. $5 A$ ) and heart function as determined by echocardiography of the $c P h d 2^{-1-}$ mice were found to be not different compared with Phd $2 w t$ mice (Fig. 5, B-D). Interestingly, a significant difference between Phd2 wt and $c P h d 2^{-1-}$ mice, however, could be observed when analyzing capillaries in the heart via CD31 immunostaining (Fig. 6). In the $c P h d 2^{-1-}$ hearts significantly more CD31-positive staining was detected. This was due to an increased capillary area but not to an increase in the number of capillaries (Fig. $6 B$ ). Taken together, basal heart structure and function are, besides an increased diameter of the capillaries, not changed in 8 weeks old mice as a consequence of a lack of PHD2 in ventricular cardiomyocytes. In this regard it should be noted that $M L C v C r e \times H I F-1 \alpha^{\text {flox/flox }}$ mice similarly do not present obvious changes in resting heart function (34). In contrast to resting conditions, however, HIF- $1 \alpha$ indeed seems to 


\section{Original Publications}

A $\mathbf{w t}_{\mathrm{w}(\mathrm{n}=3)} \mathrm{cPhd2}^{2}(\mathrm{n}=4)$
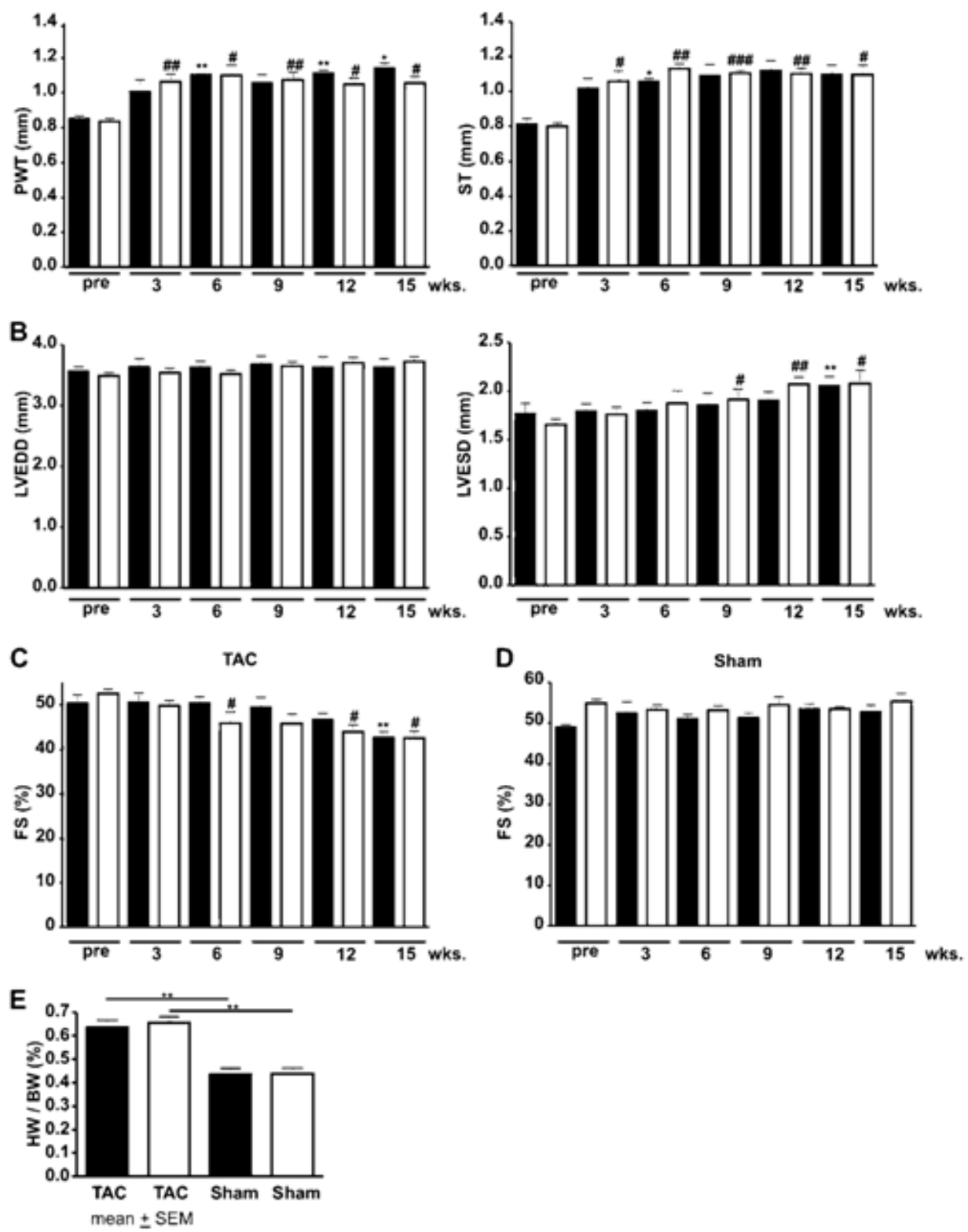

FIGURE 7. PHD2 does not affect cardiac hypertrophy and cardiac function after TAC in female mice. Sustained pressure overload was induced in 10-week-old female $P h d)$ wt and $(P h d)^{-1-}$ mice by TAC Subsequently $(A)$ posterior wall thickness $(P W T$ ) and septum thickness $(S T)$ as well as $(B)$ left ventricular end diastolic diameter (IVEDD) and left ventricular end systolic diameter (IVESD) were analyzed before (pre) and up to 15 weeks after TAC by echocardiography. Based on the left ventricular end diastolic diameter and left ventricular end systolic diameter, fractional shortenings (FS) of TAC-treated (C) and sham-operated animals $(D)$ were determined. In addition, heart weight (HW) to body weight (BW) ratios were determined 15 weeks after TAC ( $(E)$. $P<0.05$ TAC-treated $P$ hd 2 wt versus nontreated/pre Phd2 wt mice; ${ }^{*}, p<0.01$ TAC-treated Phd2 wt versus nontreated/pre Phd2 wt mice; $t, p<0.05$ TAC-treated $c$ Phd2 ${ }^{-1-}$ versus

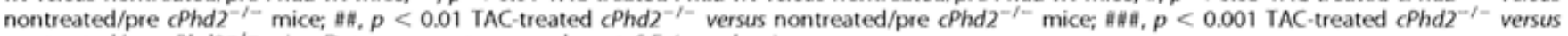
nontreated/pre $c$ Phd2 ${ }^{-1-}$ mice. Data represent mean values \pm S.E. (error bars).

play a role in the adaptation of the heart to increased mechanical load or ischemia $(20,26)$. Therefore, we analyzed the $c P h d 2^{-1-}$ mice under stressed conditions, i.e. TAC or LAD.

Lack of PHD2 in the Heart Does Not Affect the Cardiac Response to Increased Afterload -Increased mechanical load by TAC stimulates an adaptation program, which results in hypertrophy and if the load sustains in cardiac failure. We applied the aortic constriction between the branches of the truncus bra. chiocephalicus and the arteria carotis communis and followed the mice up to 15 weeks by echocardiography. As expected
TAC-treated Phd $2 w t$ and $c P h d 2^{-1-}$ mice developed cardiac hypertrophy as a consequence of the aortic constriction (Fig. $7 A)$ whereas sham-treated animal did not (data not shown). The load-induced increase in posterior wall thickness and septum thickness, however, was not different in Phd 2 wt compared with $c P h d 2^{-1-}$ mice. Likewise, both, ie. TAC-treated Phd 2 wt and $c P h d 2^{-1-}$ mice, but not the sham-treated animals (Fig. $7 D$ ), developed an increased left ventricular end systolic diameter (Fig. $7 B$ ) and a drop in FS (Fig. 7C) to a similar extent at around 12-15 weeks after the intervention. Furthermore, no change in 


\section{Original Publications}

\section{PHD2 and Myocardial Ischemia}

A
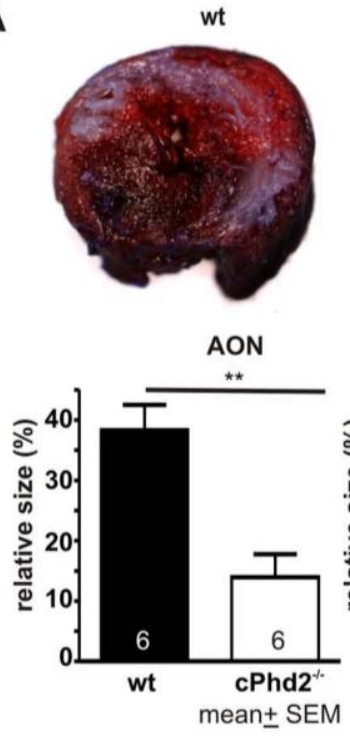

B

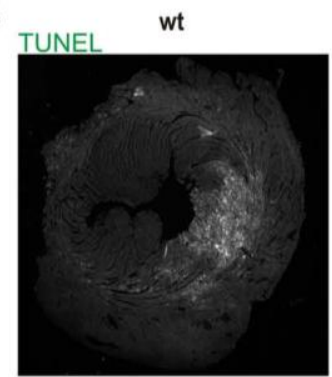

TUNEL, Hoechst
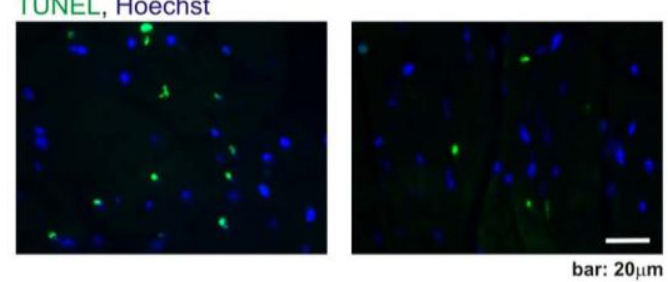

C

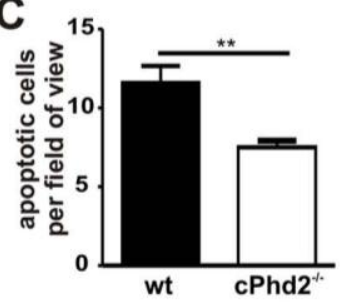

D

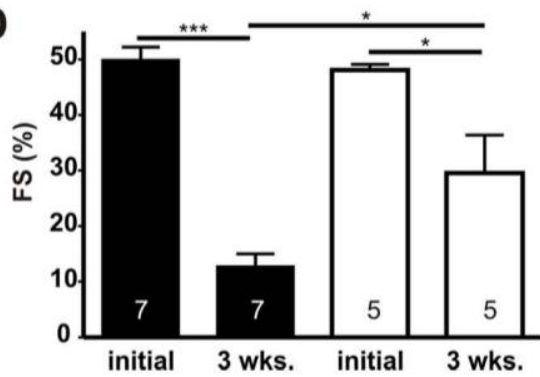

FIGURE 8. $C$ PHD2 ${ }^{-1-}$ mice are protected from myocardial infarction. $A$, hearts of female Phd 2 wt and $c P h d 2^{-1-}$ mice were harvested $6 \mathrm{~h}$ after LAD ligation, and the AON (white area) and the AAR (non-blue red area) were the heart to body weight ratio was seen in TAC-treated $P h d 2 w t$ mice compared with $c P h d 2^{-1-}$ mice (Fig. 7E).

Cardiac PHD2 Knock-out Mice Are Protected from Acute Myocardial Ischemia - Ligation of the LAD allows studying the acute and chronic pathophysiological processes in myocardial ischemia and was applied to $P h d 2 w t$ and $c P h d 2^{-1-}$ mice. The extent of tissue, which was well supplied by blood $6 \mathrm{~h}$ after the intervention, was analyzed by Evans blue perfusion. Myocardial slices were additionally stained with TTC to discriminate between nonperfused dead and vital tissue. This technique relies on the ability of dehydrogenase enzymes and co-factors in the vital tissue to react with tetrazolium salts to form a formazan pigment. Vital tissue appears red and reflects the AAR, whereas dead/nonvital tissue appears white, indicating the $\mathrm{AON}$. The AAR and the AON were significantly smaller in the $c P h d 2^{-1-}$ mice compared with littermate $P h d 2 w t$ control mice (Fig. 8A). This correlated with a significantly smaller number of apoptotic cardiomyocytes in the infarcted area in the $\mathrm{CPhd2^{-/- }}$ mice as determined by TUNEL assays (Fig. $8, B$ and $C$ ).

Echocardiography was performed at base line and 3 weeks after myocardial infarction in $c P h d 2^{-1-}$ mice compared with Phd $2 w t$ control mice (Fig. 8D). Before LAD ligation the FS was not different between $c P h d 2^{-1-}$ and control mice and was reduced in both groups after myocardial infarction. In line with the smaller AAR and AON, myocardial function was better preserved in the $c P h d 2^{-1-}$ mice as indicated by a significant higher FS 3 weeks after surgery in $c P h d 2^{-1-}$ mice than in $P h d 2$ $w t$ control mice.

\section{DISCUSSION}

Small molecule PHD inhibitors have been demonstrated to be promising in strategies for treating diseases related to hypoxic adaptation like anemia, stroke, or myocardial infarction $(14,35,36)$. The concept of cardiac tissue protection is delineated from several recent preclinical studies. Intramyocardial injection of small hairpin RNA targeting PHD2 following LAD ligation results in improved neovascularization in the peri-infarct region and improved cardiac function as indicated by determining FS (37). Treatment of mice with the PHD inhibitors dimethyloxaloylglycine or GSK60 had similar protective effects in mouse or rat myocardial infarction models $(28,38)$. Furthermore, a recent report by Hyvärinen et al. has underscored the cardiac protective effect of HIF- $1 \alpha$ in a PHD2 hypomorphic mouse model (39). Taken collectively, these studies point to a cardiac tissue protective mechanism by inhibiting

\footnotetext{
determined with Evans blue perfusion and TC staining. Representative midmyocardial cross-sections of stained hearts are shown. AAR and AON were significantly smaller in $C P H D 2^{-1-}$ hearts. The numbers in the bars indicate the number of animals analyzed. ${ }^{* *}, p<0.01$. Data represent mean values \pm S.E. (error bars). $B$, heart sections of $c P h d 2^{-1-}$ mice $(n=3)$ and wt mice $(n=3)$ were analyzed for apoptotic cells by TUNEL assay after LAD ligation. Nuclei were stained with Hoechst (blue), apoptotic nuclei were stained green by the TUNEL assay. Representative images are shown. $C$, apoptotic cells per field of view within the AAR after myocardial infarction of $\mathrm{CPhd2}^{-1-}$ and Phd2 wt mice were quantified. **, $p<0.01$. Data represent mean values \pm S.E. (error bars). $D$, cardiac function is preserved in $C P H D 2^{-1-}$ mice 3 weeks after myocardial infarction. FS was analyzed before (initial) and 3 weeks after LAD ligation by echocardiography. ${ }^{*}, p<0.05 ; * * *, p<0.001$. Data represent mean values \pm S.E. The numbers in the bars represent the number of animals analyzed.
} 


\section{Original Publications}

\section{PHD2 and Myocardial Ischemia}

PHD activity in ischemia; however, they do not explicitly answer whether inhibiting the activities of PHDs in cardiomyocytes is involved in this process. In our study, in which the surgeon and the investigator analyzing the myocardial infarct size were blinded, both, i.e. AAR and AON, were significantly decreased after LAD ligation in $c P h d 2^{-1-}$ mice, which lack PHD2 just in ventricular cardiomyocytes compared with $P h d 2$ $w t$ littermates. This correlated with a decreased number of apoptotic cells. Moreover, 3 weeks after LAD ligation $c P h d 2^{-1-}$ mice demonstrated better heart function compared with $P h d 2$ wt control animals. To this end the present study adds to the understanding of the consequences of loss of PHD2 specifically in cardiomyocytes.

In line with recently published results obtained with a mouse model similar to the one developed in our study, filament structure and cardiac function were not affected by the cardiomyocyte-specific loss of PHD2 in young mice (22). However, we did not observe any change in the response of $c P h d 2^{-1-}$ mice compared with $P h d 2$ wt mice to sustained pressure overload in regard to cardiac hypertrophy or development of heart failure. This is in contrast to the results of Moslehi et al. (22), who have described a decompensation in PHD2-deficient hearts in response to increased afterload. TAC in the mouse is a commonly used experimental model for pressure overload-induced cardiac hypertrophy and heart failure. The discrepancy in the TAC model comparing the study by Moslehi et al. and our study may be in part explained by the different deleter Cre mice applied. Furthermore, the development of cardiac failure after TAC intervention is highly depending on the severity of aortic constriction. The severity is mainly determined by the ratio of the basal diameter of the aorta and the diameter of the needle, which is used as placeholder during the constriction procedure. Whereas the needle size was the same in both studies, the severity of the constriction still seems to be more severe in the study of Moslehi et al. because the fractional shortening in the challenged mice was reduced already after $4-8$ weeks, whereas our mice responded just after $12-15$ weeks with a diminished FS.

Interestingly, we observed a significant increase in myocardial capillary size as defined by CD31 staining but not in the number of capillaries. This is consistent with the observation that genes involved in angiogenesis were not found to be up. regulated in the $c \mathrm{Phd}^{-1-}$ mice presented in this study. Still, it is conceivable that the increased capillary diameter results in an improved cardiac blood supply which might contribute to the cardiac tissue protection in the $c P h d 2^{-t-}$ mice $6 \mathrm{~h}$ after ligation of the LAD.

A HIF- $1 \alpha$-mediated cardioprotection has also been observed in cardiac-specific HIF-1 transgenic mice after myocardial infarction (40) and in ischemia-reperfusion in mice carrying a hypomorphic Phd2 allele (39) and, very recently, in $\mathrm{Phd1}^{-f-}$ mice (41). The cause of cardioprotection is likely multifactoral and due to the activation of several HIF target genes and the subsequent modulation of pathways involved in, for example, $\beta$-catenin signaling (41), the purinergic signaling pathways (28), and the glucose metabolism (39). However, in the $c P h d 2^{-l-}$ mice presented in our study purinergic signaling was not affected as no significant changes in adenosine $2 \mathrm{~B}$ receptor and CD73 transcriptional levels were observed. Yet, similar to the study by Hyvärinen et al. (39), we noticed significantly increased mRNA levels for Glut-1 and several enzymes of gly. colysis which are all known HIF targets (42). A shift from oxidative to glycolytic metabolism in the heart confers an advantage in surviving ischemic insults. This might, together with the increased capillary vessel area in $\mathrm{cPhd} 2^{-i-}$ hearts, explain the cardioprotection after acute myocardial infarction.

Taken together, our data indicate that deficiency of PHD2 in the heart does not affect the response toward increased mechanical load but induces an acute tissue protective effect in case of myocardial ischemia. Long term inhibition of PHD2 as well as long term stabilization of HIF- $1 \alpha$ in the heart seem to impair heart function as demonstrated in old (8 months) $c P h d 2^{-t-}$ mice (22) (and own observations) and heart-specific HIF-l $\alpha$ transgenic mice (21). Further studies, which clarify for how long and when in relation to the myocardial insult PHD activity needs to be diminished, are therefore needed to delineate whether the application of small molecule PHD inhibitors are feasible and useful for inducing tissue protective effects in case of myocardial infarction.

Acknowledgnents-We thank Gudrun Federkeil for help with the cryosections and Annette Hillemann for expert technical assistance.

\section{REFERENCES}

1. Schofield, C. 1., and Ratcliffe, P. 1. (2005) Biochem Biophys. Res Commut $30.617-626$

2. Wenger, R. H. (2002) FASEB J. 16. 1151-1162

3. Wang, G. L., and Semenza, G. L. (1995) J. Biok. Chess. 270, 1230-1237

4. Ivan, M., Kondo, K., Yang, H., Kim, W., Valiando, I., Ohh, M., Salic, A., Asara, I. M., Lane, W. S., and Kaelin. W. G., Ir. (2001) Science 292. $464-468$

5. laakkola, P., Mole, D. R., Tian, Y. M., Wilson, M. 1., Gielbert, 1., Gaskell, S. I., von Kriegsheim, A., Hebestreit, H. F., Mukherii, M., Schofield, C. I., Maxwell, P. H., Pugh, C. W., and Ratcliffe, P. I. (2001) Science 292. $468-472$

6. Epstein, A. C., Gleadle, I. M., McNeill, L. A., Hewitson, K. S., O'Rourke, I. Mole, D. R., Mukherii, M., Metzen, E., Wilson, M. I., Dhanda, A., Tian. Y. M., Masson, N., Hamilton, D. L., laakkola, P., Barstead, R., Hodgkin, I., Maxovell, P. H., Pugh, C. W., Schofield, C. I., and Ratcliffe, P. I. (2001) Cell $107,43-54$

7. Bruick, R. K, and McKnight, S. L. (2001) Science 294. 1337-1340

8. Hon, W. C., Wilson, M. I., Harlos, K., Claridge, T. D., Schofield, C. I., Pugh, C. W., Maxivell, P. H., Ratcliffe, P. 1., Stuart, D. 1., and lones, E. Y. (2002) Nature 417, $975-978$

9. Min, J. H., Yang, H., Jvan, M., Gertler, F., Kaelin, W. G., Jr., and Pavletich, N. P. (2002) Science 296. 1886-1889

10. Maxwell, P. H., Wiesener, M. S., Chang, G. W., Clifford, S. C, Vaux, E C., Cockman, M. E., Wykoff, C. C., Pugh, C. W., Maher, E. R., and Ratcliffe, P. 1. (1999) Nature 399, 271-275

11. Berra, E. Benizri, E., Ginouvès, A., Volmat, V., Roux, D., and Pouyssegeur. 1. (2003) EMBO I. 22, 4082-4090

12. Lieb, M. E., Menzies, K, Moschella, M. C., Ni, R, and Taubman, M. B. (2002) Biochen. Cell Biol. s0, 421-426

13. Appelhoff, R. I., Tian, Y. M., Raval, R. R., Turley, H., Harris, A. L., Pugh, C. W., Ratcliffe, P. 1., and Gleadle, I. M. (2004) J. Biol. Clown. 279. $38458-3 \& 465$

14. Katschinshi, D. M. (2009) Acta Plusiol. 195, 407-414

15. Takeda, K., Ho, V. C., Takeda, H., Duan, L. I., Nagy, A., and Fong G. H. (2006) Mol. Cell Biol. 26, $8336-8346$

16. Takeda, K. Cowan, A, and Fong, G. H. (2007) Circulation 116, 774-781

17. Minamishima, Y. A., Moslehi, 1., Bardeesy, N., Cullen, D., Bronson, R. T., and Kaelin, W. G., Ir. (2008) Blood 111.3236-3244 


\section{Original Publications}

\section{PHD2 and Myocardial /schemia}

18. Takeda, K., Aguíla, H. L., Parikh, N. S., Li, X., Lamothe, K., Duan, L. J., Takeda, H., Lee, F. S., and Fong, G. H. (2008) Blood 111, 3229-3235

19. Krishnan, J., Ahuja, P., Bodenmann, S., Knapik, D., Perriard, E., Krek, W., and Perriard, J. C. (2008) Circ. Res. 103, 1139-1146

20. Krishnan, J., Suter, M., Windak, R., Krebs, T., Felley, A., Montessuit, C., Tokarska-Schlattner, M., Aasum, E., Bogdanova, A., Perriard, E., Perriard, J. C., Larsen, T., Pedrazzini, T., and Krek, W. (2009) Cell Metab. 9, $512-524$

21. Bekeredjian, R., Walton, C. B., MacCannell, K. A., Ecker, J., Kruse, F., Outten, J. T., Sutcliffe, D., Gerard, R. D., Bruick, R. K., and Shohet, R. V. (2010) PLoS One 5, e11693

22. Moslehi, J., Minamishima, Y. A., Shi, J., Neuberg, D., Charytan, D. M., Padera, R. F., Signoretti, S., Liao, R., and Kaelin, W. G., Jr. (2010) Circulation 122, 1004-1016

23. Zhang, Y., Muyrers, J. P., Testa, G., and Stewart, A. F. (2000) Nat. Biotechnol. 18, 1314-1317

24. Percy, M. J., Furlow, P. W., Beer, P. A., Lappin, T. R., McMullin, M. F., and Lee, F. S. (2007) Blood 110, 2193-2196

25. Minamisawa, S., Gu, Y., Ross, J., Jr., Chien, K. R., and Chen, J. (1999) J. Biol. Chem. 274, $10066-10070$

26. Silter, M., Kögler, H., Zieseniss, A., Wilting, J., Schafer, K., Toischer, K., Rokita, A. G., Breves, G., Maier, L. S., and Katschinski, D. M. (2010) Eur. J. Physiol. 459, 569-577

27. Bohl, S., Medway, D. J., Schulz-Menger, J., Schneider, J. E., Neubauer, S., and Lygate, C. A. (2009) Am. J. Physiol Heart Circ Physiol. 297, H2054-2058

28. Eckle, T., Köhler, D., Lehmann, R., El Kasmi, K., and Eltzschig, H. K. (2008) Circulation 118, 166-175

29. Zieseniss, A., Schroeder, U., Buchmeier, S., Schoenenberger, C. A., van

den Heuvel, J., Jockusch, B. M., and Illenberger, S. (2007) Cell Tissue Res. $327,583-594$

30. Hirsilä, M., Koivunen, P., Günzler, V., Kivirikko, K. I., and Myllyharju, J. (2003) J. Biol. Chem. 278, 30772-30780

31. Wenger, R. H., Stiehl, D. P., and Camenisch, G. (2005) Sci. STKE 2005 , re12

32. Stiehl, D. P., Wirthner, R., Köditz, J., Spielmann, P., Camenisch, G., and Wenger, R. H. (2006) J. Biol Chem. 281, 23482-23491

33. Palazzuoli, A., Gallotta, M., Quatrini, I., and Nuti, R. (2010) Vasc. Health RiskManag, 6, 411-418

34. Huang, Y., Hickey, R. P., Yeh, J. L., Liu, D., Dadak, A., Young, L. H., Johnson, R. S., and Giordano, F. J. (2004) FASEB J. 18, $1138-1140$

35. Myllyharju, J. (2009) Curr. Pharm. Des. 15, 3878-3885

36. Yan, L., Colandrea, V. J., and Hale, J. J. (2010) Expert Opin. Ther. Pat. 20, $1219-1245$

37. Huang, M., Chan, D. A., Jia, F., Xie, X., Li, Z., Hoyt, G., Robbins, R. C., Chen, X., Giaccia, A. J., and Wu, J. C. (2008) Circulation 118, S226-233

38. Bao, W., Qin, P., Needle, S., Erickson-Miller, C. L., Duffy, K. J., Ariazi, J. L. Zhao, S., Olzinski, A. R., Behm, D. J., Pipes, G. C., Jucker, B. M., Hu, E., Lepore, J. J., and Willette, R. N. (2010) J. Cardiovasc. Phamacol. 56, $147-155$

39. Hyvärinen, J., Hassinen, I. E., Sormunen, R., Mäki, J. M., Kivirikko, K. I., Koivunen, P., and Myllyharju, J. (2010) J. Biol. Chem. 285, 13646-13657

40. Kido, M., Du, L., Sullivan, C. C., Li, X., Deutsch, R., Jamieson, S. W., and Thistlethwaite, P. A. (2005) J. Am. Coll. Cardiol, 46, 2116-2124

41. Adluri, R. S., Thirunavukkarasu, M., Dunna, N. R., Zhan, L., Oriowo, B. Takeda, K., Sanchez, J., Otani, H., Maulik, G., Fong, G. H., and Maulik, N. (2010) Antioxid. Redox Signal, in press

42. Semenza, G. L. (2007) J. Bioenerg. Biomembr. 39, 231-234

\section{Supplemental figures}

\section{Fig.1}

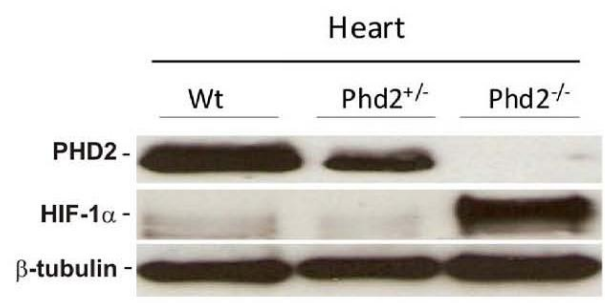

Supplementary Figure 1: Protein expression in the Phd 2 deficient heart. Hearts from mouse embryos (PGKCre $x$ Phd2flox/flox) were isolated at E14.5 and western blot analysis was performed on protein extracts (10 $\mu \mathrm{g}$ total protein). The anti mouse-Phd2 antibody targeted the C-terminal end of the protein (homemade). No PHD2 signal could be detected in the Phd2-/- heart (as in the rest of the embryo (data not shown)) resulting in the protection of HIF-1 $\alpha$ protein from degradation. $\beta$-tubulin served as loading control. 


\section{Original Publications}

\section{Fig.2}

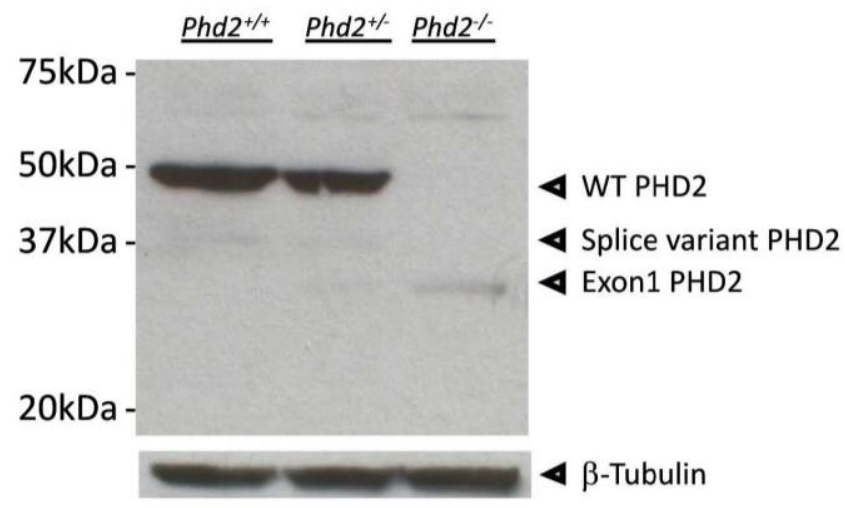

Supplementary Figure 2: Proteins were isolated from embryos (PGKCre $x$ Phd2flox/flox) at E12.5 and western blot analysis was performed on protein extracts (30 $\mu \mathrm{g}$ total protein). The anti mouse-Phd2 antibody detects the $\mathrm{N}$-terminal end of the protein (Cell Signaling). A truncated Phd2 protein (exon1) could be detected in the Phd2-\% as well as in the Phd2+/- embryos. $\beta$-tubulin served as loading control. 


\section{Original Publications}

\subsection{Unfavourable consequences of chronic cardiac HIF-1 $\alpha$ stabilization}

Kido et al. created a mouse strain carrying the unmodified HIF-1 $\alpha$ gene under the control of the $\alpha \mathrm{MHC}$ promoter (HIF-1 $\alpha(+))$ (Kido et al., 2005). By analyzing this mouse model it is possible to determine the effects of HIF-1 $\alpha$, which is normally only stabilized and therefore active in hypoxic conditions, in a normoxic environment. Kido et al. could show that HIF-1 $\alpha(+)$ mice have a better outcome after myocardial infarction as indicated by a smaller infarct size and a better preserved heart function four weeks after the ligation of the left anterior descending artery.

Long-term consequences of HIF-1 $\alpha$ stabilization and the response of these mice to mechanical load, however, were not investigated. The following manuscript describes the investigations of these HIF-1 $\alpha$ transgenic mice $\left(H i f-1 \alpha^{t g}\right)$ when challenged by transverse aortic constriction. Furthermore, a group of mice was followed over a time period of eight months to investigate long term consequences of HIF-1 $\alpha$ stabilization. In addition, the metabolism and the calcium handling of $H i f-1 \alpha^{\text {tg }}$ mice in comparison to $w t$ littermates were determined.

In summary, I was able to show that the metabolism of Hif- $1 \alpha^{\text {tg }}$ mice is shifted towards glycolysis with a net increase in glucose uptake and that furthermore $\mathrm{Ca}^{2+}$-handling is altered with increased $\mathrm{Ca}^{2+}$-transients and a faster $\mathrm{Ca}^{2+}$ re-uptake. Subjected to transverse aortic constriction Hif- $1 \alpha^{\text {tg }}$ mice exhibited profound cardiac decompensation. Moreover, cardiomyopathy was also seen in aging Hif- $1 \alpha^{\text {tg }}$ mice. 


\title{
Unfavourable consequences of chronic cardiac HIF-1 $\alpha$ stabilization
}

\author{
Marion Hölscher', Katrin Schäfer², Sabine Krull', Katja Farhat', Amke Hesse', \\ Monique Silter ${ }^{1}$, Yun Lin ${ }^{3}$, Bernd J. Pichler ${ }^{3}$, Patricia Thistlethwaite ${ }^{4}$, Ali El-Armouche ${ }^{5}$, \\ Lars. S. Maier ${ }^{2}$, Dörthe M. Katschinski ${ }^{1 * \dagger}$, and Anke Zieseniss ${ }^{1 \dagger}$
}

${ }^{1}$ Department of Cardiovascular Physiology, Universitätsmedizin, Georg-August-University Göttingen, Humboldtallee 23, 37073 Göttingen, Germany; ${ }^{2}$ Department of Cardiology and Pneumology, Georg-August-University Göttingen, Germany; ${ }^{3}$ Department of Preclinical Imaging and Imaging Technology of the Werner Siemens-Foundation, University of Tübingen, Germany; ${ }^{4}$ Division of Cardiothoracic Surgery, University of California, San Diego, CA, USA; and ${ }^{5}$ Department of Pharmacology, Georg-August-University Göttingen, Germany

Received 19 October 2011; revised 19 December 2011; accepted 12 january 2012

Time for primary review: 25 days

Aims

The hypoxia-inducible factor-1 (HIF-1) is the master modulator of hypoxic gene expression. The effects of chronically stabilized cardiac HIF-1 $\alpha$ and its role in the diseased heart are not precisely known. The aims of this study were as follows: (i) to elucidate consequences of HIF-1 $\alpha$ stabilization in the heart; (ii) to analyse long-term effects of HIF-1 $\alpha$ stabilization with ageing and the ability of the HIF-1 $\alpha$ overexpressing hearts to respond to increased mechanical load; and (iii) to analyse HIF-1 $\alpha$ protein levels in failing heart samples.

Methods In a cardiac-specific HIF-1 $\alpha$ transgenic mouse model, constitutive expression of HIF-1 $\alpha$ leads to changes in capillary and results area and shifts the cardiac metabolism towards glycolysis with a net increase in glucose uptake. Furthermore, $\mathrm{Ca}^{2+}$ handling is altered, with increased $\mathrm{Ca}^{2+}$ transients and faster intracellular $\left[\mathrm{Ca}^{2+}\right]$ decline. These changes are associated with decreased expression of sarcoplasmic/endoplasmic reticulum calcium ATPase 2 a but elevated phosphorylation of phospholamban. HIF-1 $\alpha$ transgenic mice subjected to transverse aortic constriction exhibited profound cardiac decompensation. Moreover, cardiomyopathy was also seen in ageing transgenic mice. In parallel, we found an increased stabilization of HIF-1 $\alpha$ in heart samples of patients with end-stage heart failure.

Conclusion Changes induced with transgenic cardiac HIF-1 $\alpha$ possibly mediate beneficial effects in the short term; however, with increased mechanical load and ageing they become detrimental for cardiac function. Together with the finding of increased HIF-1 $\alpha$ protein levels in samples from human patients with cardiomyopathy, these data indicate that chronic HIF-1 $\alpha$ stabilization drives autonomous pathways that add to disease progression.

Keywords Hypoxia - Prolyl-4-hydroxylase domain enzymes - Hypoxia-inducible factor-1 • Metabolism • Ischaemia • Cardiomyopathy

\section{Introduction}

Coronary artery disease and increased mechanical load are two major leading causes for cardiomyopathy. ${ }^{1}$ Myocardial ischaemia, in the case of coronary artery disease, and cardiac hypertrophy, in the case of increased mechanical load, are both associated with a dysfunctional oxygen supply to the heart. This is due to vessel occlusion and capillary rarefaction, respectively. ${ }^{2}$ Hypoxia is the initial trigger during myocardial ischaemia, and during increased mechanical load Hypoxia is the initial trigger during the process of myocardial remodelling.
The hypoxia-inducible factor (HIF) plays a pivotal role in the transcriptional response to changes in oxygen availability. ${ }^{3}$ HIF comprises two subunits, the $\alpha$-subunit, which is regulated in an oxygendependent manner, and the constitutively expressed $\beta$-subunit. The $\mathrm{HIF} \alpha$ subunit has an exceptionally short half-life and low steady-state levels in normoxic conditions. ${ }^{4}$ The regulation of HIF $\alpha$ half-life is mediated by three prolyl-4-hydroxylase domain (PHD) enzymes, which hydroxylate two prolyl residues by the use of molecular oxygen. ${ }^{5,6}$ Hydroxylation of HIF $\alpha$ allows binding of the von HippelLindau tumour ( $\mathrm{PVHL}$ ) suppressor, which targets HIF $\alpha$ for

\footnotetext{
* Corresponding author. Tel: +4955139 9778; fax: +4955139 5895, Email: doerthe.katschinsk@@med.uni.goettingen.de

tThese authors contributed equally to this work.

Published on behalf of the European Society of Cardiology. All rights reserved. (C) The Author 2012. For permissions please email: journals.permissions@oup.com.
} 


\section{Original Publications}

proteasomal degradation. ${ }^{78}$ Hypoxia impairs the hydroxylation, which results in $\mathrm{HIF} \alpha$ stabilization, nuclear accumulation, heterodimerization with HIF-1 $1 \beta$, and subsequent hypoxia-inducible gene expression. HIF-1 is known to control the expression of a myriad of genes that regulate cell survival, cell metabolism, and angiogenesis in hypoxic conditions. ${ }^{9}$ In line with these documented functions, increasing HIF-1 $\alpha$ stabilization has been reported to be protective against acute cardiac ischaemia. ${ }^{10-12}$ The consequences of HIF-1 $\alpha$ stabilization in the heart, which may form the basis for ischaemic cardioprotection, are not fully characterized. ${ }^{13}$

In particular, the consequences of long-term stabilization of HIF- $1 \alpha$ in the heart are yet to be described. To clarify the outcome of chronically increased HIF-1 $\alpha$ protein levels in the heart, we analysed the influence of cardiac transgenic overexpression of HIF-1 $\alpha$ in mice ( $H$ if $-1 \alpha^{\mathrm{tg}}$ ). It has been shown previously that cardiac HIF- $1 \alpha$ stabilization has tissue protective effects when these mice are challenged by myocardial infarction at a young age (12-week-old mice) $i^{14}$ however, the long-term consequences of HIF- $1 \alpha$ stabilization in older mice and their response to mechanical load are unknown.

\section{Methods}

\subsection{Animals and surgical intervention}

All protocols regarding animal experimentation were approved by the Niedersächsische Landesamt für Verbraucherschutz und Lebensmittelsicherheit (33.9.42502-04-10/0024 and 33.9.42502-04-10/0069) and conform with the Directive 2010/63/EU of the European Parliament Surgical interventions were performed with littermate mice that were either wild-type (Hif-1 $\alpha^{\mathrm{m}}$ ) or heterozygous for the Hilf-1 $\alpha$ transgene $\left(H i f-1 \alpha^{\text {tg }}\right)$ as described previously: ${ }^{14}$ see Supplemental Methods for further details.

\subsection{Echocardiography}

Echocardiography and measurements of posterior wall thickness (PWT), septum thickness (ST), left ventricular end-systolic diameter (LVESD), left ventricular end-diastolic diameter (LVEDD), and fractional shortening (FS) of ageing mice were performed as described before by our group. ${ }^{15}$ Assessment of fractional area shortening (FAS), PWT, ST, LVESD, and LVEDD after transverse aortic constriction (TAC) was performed using a Vevo2100 system (VisualSonics, Toronto, ON, Canada). See Supplemental Methods for further details.

\subsection{Positron emission tomography analysis}

The $\left[{ }^{18} \mathrm{~F}\right] \mathrm{FTHA}$ and $\left[{ }^{18} \mathrm{~F}\right] \mathrm{FDG}$ positron emission tomography (PET) measurements were performed on a dedicated small animal Inveon PET scanner (Siemens Healthcare, Knoxville, TN, USA) with 2 days in between to monitor the energy alteration of fatty acid oxidation and glucose metabolism. Experimental details of the PET analysis and the radiotracer production can be found in the Supplemental Methods section.

\subsection{Histology}

For Massons's Trichrome and periodic acid-Schiff staining, heart tissue was fixed in $4 \%$ paraformaldehyde in phosphate-buffered saline (PBS) and embedded in paraffin before sectioning. To visualize triglycerides and lipids. 10- $\mu$ m-thick cryosections were prepared and stained with Sudan III. Nuclei were stained with Haematoxylin.

\subsection{Immunohistochemistry}

Paraffin-embedded sections were immunostained using the CSA II System (Dako, Carpinteria, CA USA) as described previously by our group. ${ }^{11}$ The anti-HIF-1 $\alpha$ primary antibody (Novus NB100-123) was used at a 1:1000 dilution.

\subsection{Cryosections and immunofluorescence labelling}

Freshly isolated hearts were treated and stained as described before. ${ }^{16}$ Details can be found in the Supplemental Methods section.

\subsection{RNA extraction and RT-PCR analysis}

After RNA extraction, reverse transeription was performed with $2 \mu \mathrm{g}$ of RNA and a first strand complementary DNA (CDNA) synthesis kit (Fermentas $\mathrm{GmbH}$, St Leon-Rot, Germany). mRNA levels were quantified by using $0.5 \mu \mathrm{L}$ of the CDNA reaction and the SyBR Green qPCR reaction kit (Agilent, Santa Clara, CA, USA) in combination with the MX3005P light cycler (Stratagene, Santa Clara, CA, USA). The initial template concentration of each sample was calculated by comparison with serial dilutions of a calibrated standard. Primer sequences can be found in the Supplemental Methods section.

\subsection{Western blot}

Heart tissue was rapidly homogenized in a buffer containing $4 \mathrm{M}$ urea, $140 \mathrm{mM}$ Tris ( $\mathrm{pH} 6.8$ ), $1 \%$ SDS, $2 \% \mathrm{NP}-40$ and protease inhibitors (Roche, Grenzach, Germany). For immunoblot analysis, protein samples were resolved by SDS-PAGE and transferred onto nitrocellulose membranes (Amersham Biosciences, München, Germany) by semi-dry blotting (PeqLab, Erlangen, Germany). Membranes were probed with specific antibodies (see Supplemental Methods).

\subsection{Isolation of cardiomyocytes and measurement of cardiomyocyte shortening and intracellular $\mathrm{Ca}^{2+}$}

Hearts were excised from mice that were anaesthetized in an anaesthetic induction chamber with isoflurane and mounted on a Langendorff perfusion apparatus. The ventricular tissue was digested enzymatically, and single myocytes were isolated. After $\mathrm{Ca}^{2+}$ reintroduction (stepwise increase to $0.8 \mathrm{mM}$ ), myocytes were then plated onto superfusion chambers and used for immediate measurements. Myocytes were loaded with fluo-3. The dye was excited and emitted fluorescence measured. From the raw fluorescence, $\triangle F F F_{0}$ (increase in fluorescence compared to baseline) was calculated. Myocytes were field stimulated (voltage $25 \%$ above threshold) at $0.5,1,2$ and $4 \mathrm{~Hz}$ at $37^{\circ} \mathrm{C}$ until a steady state was achieved; only those cells exhibiting stable steady-state contractions were included in the study. At least 25 myocytes from four mice per group were studed. For the measurements of cardiomyocyte shortening and $\mathrm{Ca}^{2+}$ transients, the investigator was blinded regarding genotypes. For additional details see Supplemental Methods.

\subsection{Flow cytometry analysis}

Seven days after sham surgery or TAC surgery, left ventricles were isolated and sliced into small pieces. Tissue digestion, staining procedure, and subsequent flow cytometry analysis are described in the Supplemental Methods.

\subsection{Human myocardial tissue}

Failing left ventricular (LV) tissues were obtained from patients with endstage heart failure [dilated cardiomyopathy (DCM, $n=9$ ) and ischaemic cardiomyopathy (ICM, $n=10)]$ undergoing heart transplantation. The LV ejection fraction was $<30 \%$, cardiac index $1.5-3.0 \mathrm{~L} / \mathrm{min} \times \mathrm{m}^{2}$. Eight non-failing LV tissues (NF) from organ donors that could not be 


\section{Original Publications}

transplanted for technical reasons were used as controls. Donor patient histories or echocard ography revealed no signs of heart disease. Details have been published previously. ${ }^{17}$ The study conforms to the principles outlined in the Declaration of Helsinki and was reviewed and approved by the Ethical Committee of the University Hospital Hamburg (Az 532/ 116/9.7.1991)

\subsection{Statistical analyses}

Data are presented as means \pm SEM. We determined statistical differences by two-tailed Student's t-test (Figures $1 B, C$, and $G ; 2 A, C$, and $D$; $3 A, B, D$, and $E: 4 A, D$, and $r$, and $5 B$; see Supplementary material online, Figure $S 2 C-E$ and $S 3$ ), one-way ANOVA (see Supplementary material online, Figure $S 2 A$ and $B ;$ and Figure $5 H$ ) and two-way ANOVA (Figures $4 F-H, 5 A$; and $5 C-G$ ). ANOVAs were followed by Bonferroni post hoc tests. A P-value less than 0.05 was considered statistically significant.

\section{Results}

\subsection{Cardiomyopathy is associated with increased protein levels of HIF-1 $\alpha$}

In acute ischaemia, HIF-1 $\alpha$ is stabilized in the heart. ${ }^{18}$ To determine whether the HIF system is still affected in prolonged heart failure, we analysed HIF-1 $\alpha$ protein levels in ventricular tissue samples of patients with ICM and DCM and samples of non-failing hearts. In DCM samples, we found increased HIF-1 $\alpha$ protein levels compared with NF heart samples. In ICM samples, the increase was subtle and not significant (Figure $1 A$ and $B$ ). Taken together with the previously described increased HIF-1 $\alpha$ protein levels in HCM patients, ${ }^{19}$ these data indicate a chronic activation of the HIF pathway in some heart failure patients.

\subsection{Cardiac-specific Hif-1 $\alpha^{\text {tg }}$ mice have normal heart function in the resting state}

To dissect the impact of elevated HIF-1 $\alpha$ expression in the heart, we analysed the consequences of transgenic cardiac overexpression of HIF- $1 \alpha$ in a mouse model. Hif $1 \alpha^{\mathrm{g}}$ mice demonstrate significantly increased HIF-1 $\alpha$ RNA and protein levels in the heart, as demonstrated by quantitative real-time RT-PCR, western blot and immunohistochemistry analyses (Figure $T C-E$ ). Transverse Masson's Trichrome- and Sirius Red stained heart sections of 2-month-old Hif $1 \alpha^{48}$ mice did not reveal any obvious signs of gross malformation or fibrosis (Figure $1 F$ and Supplementary material online, Figure S1). Likewise, heart weight related to body weight did not show any difference when $\mathrm{Hif}-1 \alpha^{\mathrm{tg}}$ mice were compared with their wild-type littermates (Figure 1G). This was also verified by the analysis of septum thickness and posterior wall thickness by echocardiography. In addition, there was no significant change in fractional shortening, heart rate (see Supplementary material online, Figure S2A), or blood pressure (see Supplementary material online, Figure S2B).

\subsection{Cardiac-specific Hif- $1 \alpha^{\text {tg }}$ mice show differences in the expression of genes related to vasotonus, anaerobic metabolism and $\mathrm{Ca}^{2+}$ handling}

HIF-1 affects diverse pathways associated with cellular and systemic adaptation to hypoxic conditions, including oxygen supply and metabolism, which are important for HIF-mediated tissue-protective effects. ${ }^{9}$ Oxygen supply via the bloodstream is affected by vessel density and vessel diameter. Most interestingly, we found a significant up-regulation of the vascular endothelial growth factor in the hearts of the Hif $1 \alpha^{\text {tg }}$ mice only, whereas the expression of angiopoietin 1 and 2 was unaffected (Figure 2A). In contrast, we detected several HIF-1 target genes involved in regulating vascular tone, i.e. adrenomedullin, apelin, CD73, and inducible nitric oxide synthase to be up-regulated in the hearts of the $H$ Hif $1 \alpha^{\text {tg }}$ mice. Correspondingly, we found no change in the number of capillaries when analysing cryosections of left ventricles obtained from thif $1 \alpha^{\text {tg }}$ and Hif $1 \alpha^{\text {wh }}$ mice by $C D 31$ staining (Figure $2 B$ and $C$ ); however, when analysing capillary area we found a significant increase in the Hif $-1 \alpha^{48}$ mice (Figure 2D). The expected improvement in oxygen supply might be at least one reason for the cardioprotective effect following a myocardial infarction that was previously described in Hif $1 \alpha^{t 8}$ mice. ${ }^{14}$

Cellular metabolism is changed dramatically in response to hypoxia, with the net effect of increased anaerobic glycolysis. This has been attributed partly to HIF-mediated transcription of glucose transporter-1 (Glut-1) and glycolytic genes accompanied by $\mathrm{pH}$ buffering through the hypoxia-inducible carboanhydrase $\mathrm{IX}$ (CAIX). ${ }^{3}$ In line with those findings, we observed an increased mRNA expression of Glut-1, phosphofructokinase I (Pfkl) and CAIX in the Hif $1 \alpha^{48}$ hearts (Figure 3A). To determine whether this was accompanied by changes in the metabolic footprint, we performed cardiac $\left[{ }^{18}\right.$ FFDG PET analysis. Compared with their wildtype littermates, hearts of $H$ if $1 \alpha^{\text {tg }}$ mice used more glucose (Figure 3B), without changes in blood glucose and insulin levels (see Supplementary material online, Figure $S 2 C$ and D). When exposing tumour cells to hypoxia, increased anaerobic glucose metabolism is paradoxically associated with increased glycogen storage. ${ }^{20}$ Unlike the situation in tumour tissue, we could not find an increased expression of glycogen synthase 1 (Gys 1 ) and only a 1.5-fold increase in glycogen branching enzyme 1 (GBE1; Figure 3A). Moreover, there were no signs of increased glycogen storage in the hearts of $\mathrm{Hif}-1 \alpha^{\mathrm{tg}}$ animals (Figure 3C). When analysing lipid metabolism as determined by quantifying the expression of peroxisome proliferator-activated receptor $\alpha$ (PPAR $\alpha)$ and PPAR $\gamma$ and the expression of the PPAR $\gamma$ target gene product, fatty acid translocase (FAT), we found a weak yet significant down-regulation of PPAR $\alpha$, but no transcriptional changes in PPARy and FAT (Figure 3D). A slight downregulation in fatty acid metabolism in $H i f-1 \alpha^{\text {tg }}$ mice was seen when performing cardiac $\left[{ }^{18} \mathrm{~F}\right.$ FTHA PET analysis (Figure $3 E$ ). This was not significant and exhibited much lower standard uptake values (SUV) than $\left[{ }^{18} \mathrm{~F}\right] \mathrm{FDG}$ PET images of the myocardium. Sudan III staining revealed no signs of lipid accumulation in Hif $-1 \alpha^{\text {tg }}$ mice (Figure 3F). Taking these results together, the $H$ if $f \alpha^{\text {tg }}$ hearts demonstrate metabolic remodelling with an increase in glucose utilization.

Heart function relies critically on proper calcium handling. There is a recent report demonstrating that HIF- $1 \alpha$ impairs the promoter activity of the sarcoplasmic calcium pump, sarcoplasmic/endoplasmic reticulum calcium ATPase 2a (SERCA2a). ${ }^{21}$ These in vitro data were verified in our in vivo mouse model, because we observed a reduction of RNA and protein levels of SERCA2a in the Hif $-1 \alpha^{\text {tg }}$ hearts when compared with their littermates (Figure 4A, B and D). Besides SERCA2a, calcium handling critically involves the function of ryanodine receptor 2 (RyR2) and phospholamban (PLB); thus, we also investigated possible changes of these calcium handling 


\section{Original Publications}

proteins. RyR2 expression (Figure 4B) and PLB protein levels (Figure $4 C$ ) were unchanged. However, the phosphorylation level of PLB at the protein kinase A (PKA) phosphorylation site (Ser16) and the $\mathrm{Ca}^{2+} /$ calmodulin-dependent protein kinase II (CaMKII) phosphorylation site (Thr17) was elevated in the Hif $1 \alpha^{\text {tg }}$ hearts by 60 and $61 \%$, respectively (Figure $4 C$ and D). These changes were not associated with increased CaMKII protein levels or increased CaMKII activity as determined by analysing the phosphorylation state of CaMKII (Figure 4E).

To investigate whether the observed changes in SERCA2a protein level and PLB activity result in abnormal $\mathrm{Ca}^{2+}$ handling, we determined $\mathrm{Ca}^{2+}$ dynamics of isolated cardiomyocytes. Hif $-1 \alpha^{\text {tg }}$ cells demonstrated significantly increased intracellular

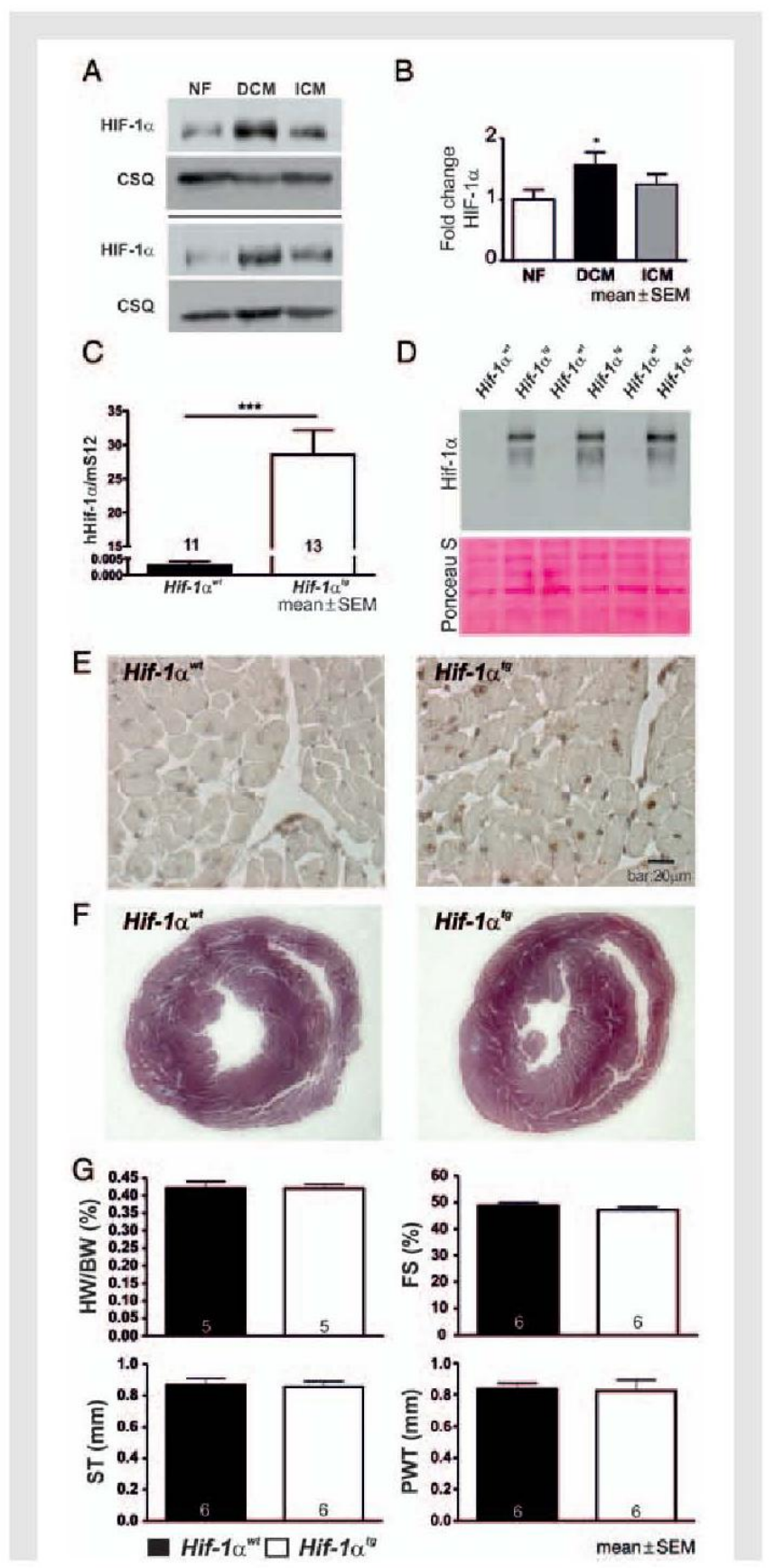

$\mathrm{Ca}^{2+}$ transients when stimulated at $0.5-4 \mathrm{~Hz}$ (Figure 4F). Twitch relaxation and intracellular $\left[\mathrm{Ca}^{2+}\right]$ decline, which are approximately $90 \%$ dependent on SERCA function in mice, ${ }^{22}$ were significantly increased and thus faster in cardiomyoctes isolated from transgenic vs. wild-type mice (Figure $4 G$ and $H$ ). Yet, the sarcoplasmic reticulum $\mathrm{Ca}^{2+}$ load did not differ $(P>0.05)$ when comparing isolated cardiomyocytes from both genotypes (Figure 4i). Overall, these data indicate that chronic overexpression of HIF-1 $\alpha$ alters $\mathrm{Ca}^{2+}$ handling properties of the myocardium, and that alterations in PLB phosphorylation may functionally override a reduction in SERCA2a protein levels.

\subsection{Hif-1 $\alpha^{\text {tg }}$ transgenic mice develop heart failure with advanced age, or at a young age when challenged by increased mechanical load}

The alterations identified in the Hif $1 \alpha^{\text {tg }}$ mice regarding vessel area, metabolism, and calcium handling are at least partly protective adaptive mechanisms, which can be interpreted as improved oxygen supply and energy-saving mechanisms in response to ischaemia. Indeed, similar mechanisms do occur as compensatory mechanisms during the development of ischaemic heart failure. ${ }^{23,24}$ Despite those changes, Hif $-1 \alpha^{\text {tg }}$ mice demonstrated no decrease in heart function at the age of 3 months, as described above. To determine whether chronic activation of the HIF-induced adaptive pathways alters heart function over time, we followed Hif- $1 \alpha^{\text {tg }}$ and Hif- $1 \alpha^{\text {wt }}$ littermates over a period of 8 months (Figure 5A). With age, Hif $-1 \alpha^{\text {tg }}$ mice developed a spontaneous increase in septum wall thickness and a decrease in fractional shortening, indicating that over time the HIF-1 $\alpha$-mediated changes result in cardiomyopathy.

Cardiac decompensation can be triggered by increased mechanical load. Initially, pressure overload leads to a compensatory response and myocardial remodelling, with a development of cardiac hypertrophy and ventricular dilatation. Ultimately, these changes have a det-

Figure I HIF-1 $\alpha$ accumulates in human DCM samples. Cardiacspecific HIF-1 $\alpha$ transgenic mice show no gross structural changes and have normal heart function in the resting state. (A) HIF-1 $\alpha$ protein was detected by western blot analysis. Tissue homogenates were prepared from normal, non-failing hearts (NF), and hearts of patients with end-stage dilated cardiomyopathy (DCM) and ischaemic cardiomyopathy (ICM). Samples were probed for HIF-1 $\alpha$ and calsequestrin (CSQ). (B) Bar graph shows mean values ( \pm SEM) from densitometric analysis of all hearts analysed in $A$. Protein levels in DCM and ICM hearts are expressed as fold change relative to protein levels in NF, which was set to $1(* P<0.05)$. (C) RT-PCR analysis confirmed the significant increase of HIF-1 $\alpha$ mRNA transcripts in the left ventricles of Hif-1 $\alpha^{\text {tg }}$ mice compared with Hif $1 \alpha^{\text {wt }}$ littermates (***p<0.001). (D) Protein extracts prepared from left ventricles of Hif- $1 \alpha^{\mathrm{wt}}$ and Hif- $1 \alpha^{\text {tg }}$ mice were analysed with anti-HIF-1 $\alpha$ antibodies, confirming stabilization of HIF-1 $\alpha$ protein in transgenic animals. $(E)$ Immunohistochemical analyses of hearts from Hif- $1 \alpha^{\text {wt }}$ and Hif-1 $\alpha^{\text {tg }}$ mice. $(F)$ Paraffin-embedded tissue sections of Hif- $1 \alpha^{\text {wt }}$ and Hif- $1 \alpha^{\text {tg }}$ hearts stained with Trichrome. $(G)$ Hearts of 8-week-old female Hif- $1 \alpha^{\text {wt }}$ and Hif-1 $\alpha^{\text {tg }}$ mice were excised, and the ratios of heart weight (HW) to body weight (BW) were determined. Septum thickness (ST), posterior wall thickness (PWT) and fractional shortening (FS) were analysed by echocardiography. 


\section{Original Publications}

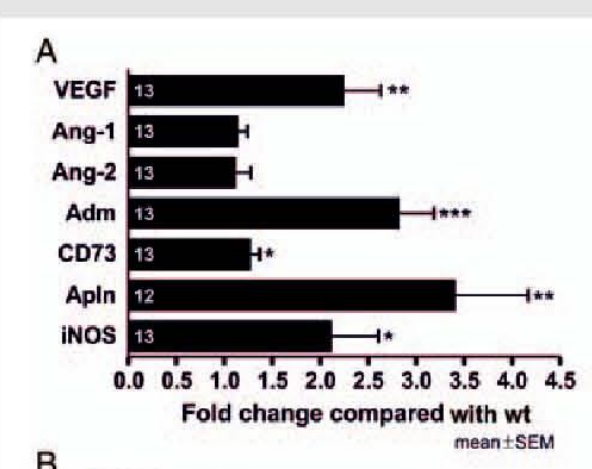

B
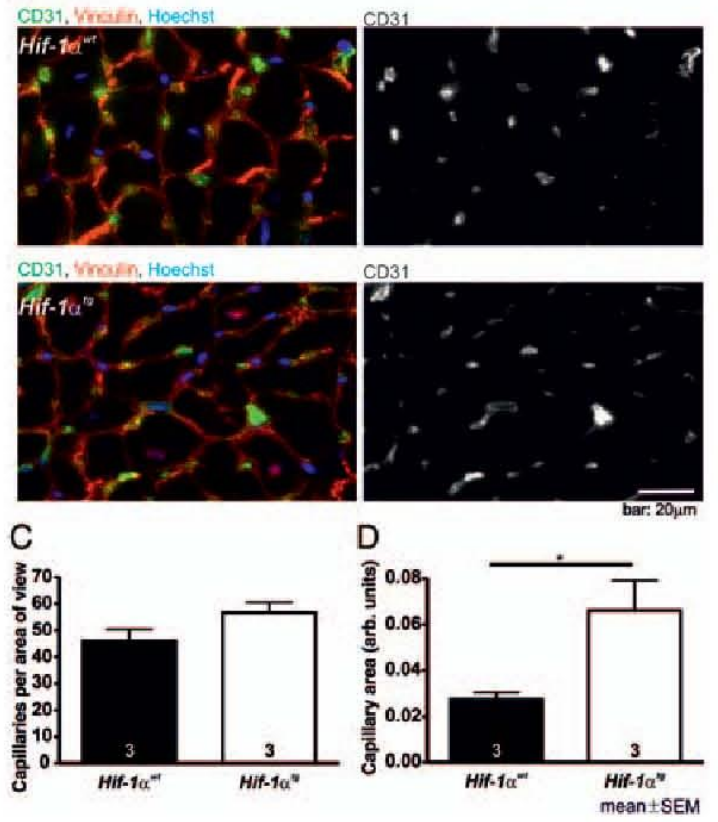

Figure 2 Vascular changes in the hearts of Hif- $1 \alpha^{\text {tg }}$ mice. (A) Quantitative real-time RT-PCR analysis of left ventricles from 8-week-old Hif-1 $\alpha^{\text {wt }}$ and Hif-1 $\alpha^{\text {tg }}$ mice was performed. Transcript levels of genes involved in angiogenesis and vasotonus regulation were analysed $\left(* P<0.05 ; * * P<0.01\right.$; $\left.{ }^{* * * P}<0.001\right)$. (B) Capillaries of hearts of Hif- $1 \alpha^{\text {wt }}$ and Hif- $1 \alpha^{\text {tg }}$ mice littermates were analysed by anti-CD31 staining. Sections were co-stained for vinculin and DNA (Hoechst). The number of capillaries were counted (C), and the capillary area $(D)$ was determined by measuring the area of CD31-stained capillaries using Imagej $(* P<0.05)$.

rimental effect on ventricular function, resulting in heart failure. We investigated the response of 2 -month-old $H i f-1 \alpha^{\text {tg }}$ mice and Hif $1 \alpha^{\text {wt }}$ littermates to increased mechanical load after TAC. Constriction of the aorta resulted in a significantly increased pressure afterload, which was not different between $H i f-1 \alpha^{\text {ts }}$ mice and Hif $1 \alpha^{\text {wt }}$ groups (Figure 5B). Cardiac HIF- $1 \alpha$ protein levels were not obviously changed 8 weeks after TAC treatment in Hif $1 \alpha^{\text {tg }}$ mice and $H$ if $-1 \alpha^{\mathrm{wt}}$, as detected by western blotting (see Supplementary material online, Figure S3A). Myocardial inflammation in sham-operated and TAC-treated animals did not show any differences when Hif $-1 \alpha^{\text {ts }}$ mice were compared with their wild-type littermates, as determined by quantifying the number of CD45-positive cells (see
Supplementary material online, Figure S3B). Left ventricular hypertrophy was indicated by a significant increase in ST and PWT in TACtreated Hif $1 \alpha^{\text {tg }}$ mice (Figure $5 C$ and D) 2 weeks after surgery, as determined by echocardiography. While Hif- $1 \alpha^{\text {wt }}$ mice did not show any signs of decompensation compared with sham-treated mice, the Hif $1 \alpha^{\text {tg }}$ mice demonstrated a significant increase in LVESD 4 and 8 weeks after surgery (Figure 5E), together with a decrease in FAS (Figure 5F). The LVEDD (Figure 5G) was not significantly affected in TAC-treated transgenic mice compared with wild-type animals. In support of the TAC-induced hypertrophy and heart failure in $H$ if $-1 \alpha^{\text {tg }}$ mice, the heart-to-tibia length ratio was also increased (Figure 5H). Taking these results together, chronic activation of HIF-1 triggers intrinsic pathways in the cardiomyocytes, which impair long-term preservation of heart function and weaken adaption to increased mechanical load.

\section{Discussion}

There is ample evidence that HIF-1 protects the heart from an acute ischaemic insult. Based on the characterization of the Hif $-1 \alpha^{\text {tg }}$ hearts, as demonstrated in our study, this may be attributed to an increased capillary area in the heart and metabolic preconditioning via increasing gene expression and glucose metabolism that is necessary for the anaerobic metabolic switch. Moreover, the changes in calcium handling in Hif- $1 \alpha^{\text {tg }}$ hearts demonstrate energy-saving mechanisms regarding the depressed SERCA2a expression. The decrease in SERCA2a might be counter-regulated by an increased PLB phosphorylation and thus, a net increase in calcium release and shorter relaxation time. Although the HIF-mediated adaptation pathways regarding metabolic reprogramming and altered calcium handling are beneficial to withstand acute ischaemia, these same pathways are indicative of defective cardiac homeostasis in the failing heart. ${ }^{11,24,25}$ Accordingly, the tissue-protective properties of HIF-1 in the young Hif $1 \alpha^{\text {ts }}$ mice were accompanied by development of heart failure with ageing or after increased mechanical load. Thus, it is tempting to speculate that chronic activation of HIF-1 and its associated adaptive pathways alone are sufficient to induce cardiomyopathy. We found increased protein levels of HIF- $1 \alpha$ in heart samples of cardiomyopathy patients, indicating that the HIF pathway is activated during disease progression. According to the data obtained with our mouse model, induction of this regulatory pathway may be beneficial initially, however, if sustained it may actively contribute to heart failure.

Bao et $a .^{26}$ showed in a rat model that treatment with GSK360A, and thus chronic stabilization of HIF-1 $\alpha$ signalling, after myocardial infarction improved cardiac remodelling and ventricular performance in the ischaemic myocardium without affecting infarct size. This is contrary to the observed cardioprotective effect in the Hif- $1 \alpha^{\text {tg }}$ mice, which goes along with a decreased infarct size. In the study of Bao et al. no long-term maladaptive effects were observed 3 months after treatment. Nevertheless, it should be noted that the animals were treated systemically, whereas in the Hif $-1 \alpha^{\text {tg }}$ mice HIF- $1 \alpha$ is stabilized in cardiomyocytes only. Furthermore, treatment with the PHD-inhibiting compound GSK360A lasted for only 4 weeks, after which the compound was withdrawn and washed out.

Likewise, the cardiac short hairpin RNA PHD2 knockdown after myocardial infarction mediates HIF-1 $\alpha$ up-regulation and improved cardiac function ${ }^{27}$ without any chronic adverse effects. Again, however, in that mouse model the HIF- $1 \alpha$ protein levels returned to baseline only 4 weeks after short hairpin RNA injection, whereas 


\section{Original Publications}

A

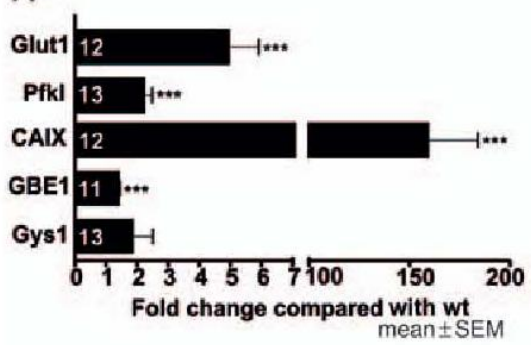

B

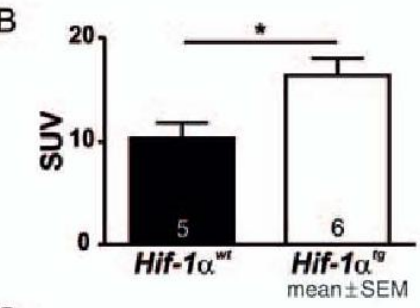

C

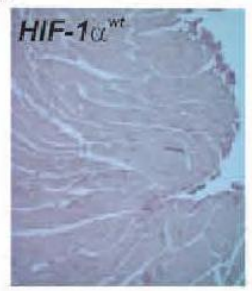

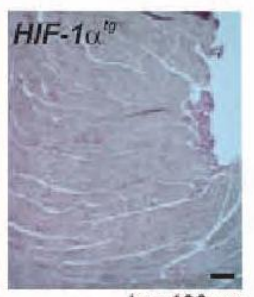

bar $100 \mu \mathrm{m}$
D

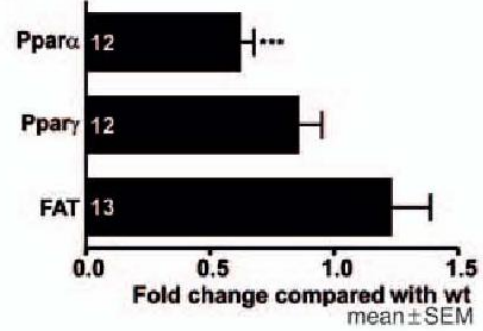

E
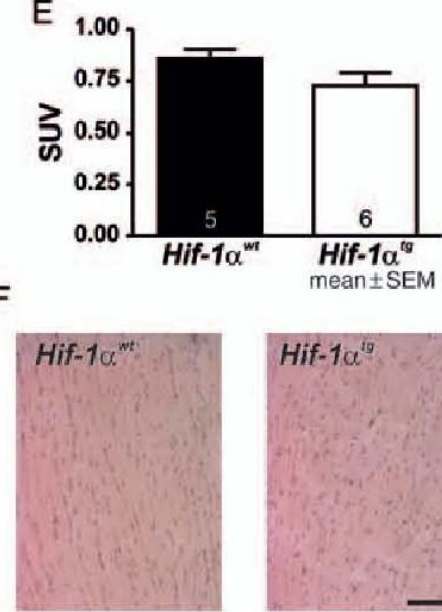

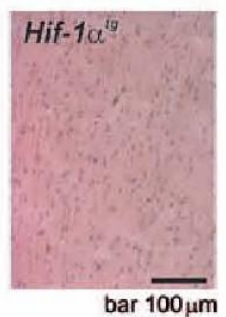

Figure 3 Metabolic changes in Hif-1 $\alpha^{\text {tg }}$ mice. (A) Quantitative real-time RT-PCR analysis of left ventricles from 8-week-old Hif-1 $\alpha^{\text {wt }}$ and Hif-1 $\alpha^{\text {tg }}$ mice was performed. Transcript levels of genes involved in glucose metabolism and pH regulation were analysed (***p $<0.001)$. (B) $\left.{ }^{18} \mathrm{~F}\right] \mathrm{FDG}$ uptake in Hif- $1 \alpha^{\text {wt }}$ and Hif- $1 \alpha^{\text {tg }}$ mice. Bar graph shows cardiac standard uptake values (SUV) at $60 \mathrm{~min}(* P<0.05)$. Data represent mean values \pm SEM. (C) Periodic acid-Schiff staining of paraffin-embedded tissue sections of Hif- $1 \alpha^{\text {wt }}$ and Hif- $1 \alpha^{\text {tg }}$ hearts. (D) RNA expression levels of PPAR $\alpha$, PPAR $\gamma$, and FAT in left ventricles from 8-week-old Hif-1 $\alpha^{\mathrm{wt}}$ and Hif-1 $\alpha^{\mathrm{tg}}$ mice as determined by quantitative real-time RT-PCR. (E) $\left[{ }^{18} \mathrm{~F}\right] \mathrm{FTHA}$ uptake in Hif-1 $\alpha^{\mathrm{wt}}$ and Hif- $1 \alpha^{\text {tg }}$ mice. Bar graph shows cardiac SUV at $60 \mathrm{~min}$. (F) Sudan III staining of frozen sections of Hif-1 $\alpha^{\text {wt }}$ and Hif- $1 \alpha^{\text {tg }}$ mice.

in the present $H$ if $-1 \alpha^{\text {tg }}$ mice we saw the first signs of cardiomyopathy at 3 months of age.

A detrimental effect associated with long-term stabilization of HIF-1 $\alpha$ is similar to ventricular dysfunction of the heart in cardiacspecific $\mathrm{pVHL}^{-1-}$ mice. ${ }^{28}$ It is important to note that a detrimental cardiac effect was also reported in tetracycline-inducible cardiacspecific HIF- $1 \alpha$ transgenic mice and cardiac-specific HIF- $2 \alpha$ transgenic mice. ${ }^{29,30}$ In both mouse models, the authors demonstrated a spontaneous cardiac decompensation of the mice at a young age, whereas the animals in our mouse model demonstrated normal heart function in non-challenged conditions but aggravated myocardial dysfunction over time and after TAC. This development of cardiomyopathy over time enabled us to determine the HIF-dependent adaptive changes in the heart, as described above, which occur before the onset of heart failure. While Bekeredjian et al. have created tetracycline-inducible HIF-1 $\alpha$ overexpressing mice, we analysed an unregulated HIF-1 $\alpha$ transgenic mouse model, which most probably presents a lower transgenic HIF-1 $\alpha$ expression compared with the acutely inducible model. ${ }^{29}$ This assumption is additionally supported by the fact that in the tetracycline-inducible model, an HIF-1 $\alpha$ variant was used that substituted amino acids critical to HIF-1 $\alpha$ degradation (i.e. Pro402Ala, Pro564Ala, and Asn803Ala), whereas in the present model the wild-type HIF-1 $\alpha$ protein was overexpressed. Likewise, Moslehi et al. have created a cardiac-specific HIF-2 $\alpha$ transgenic mouse model, which overexpresses a non-hydroxylatable HIF- $2 \alpha$ variant. $^{30}$ The different degrees of HIF $\alpha$ levels over time may explain the varying onset of heart failure in our model compared with the tetracycline-inducible HIF- $1 \alpha$ model and the HIF- $2 \alpha$ transgenic mice. Interestingly, we previously showed that mice with reduced HIF-1 $\alpha$ levels (HIF-1 $1 \alpha^{+1-}$ mice) also develop heart failure after TAC. ${ }^{15}$ This might imply that HIF- $1 \alpha$ protein levels need to be tightly regulated, whereby neither decreased nor elevated HIF-1 $\alpha$ protein levels can be tolerated.

While ischaemia develops rapidly in myocardial infarction, tissue hypoxia supposedly develops over a longer period of time during cardiac hypertrophy. The heart is particularly susceptible to hypoxia/ischaemia, because only limited reserves of high-energy phosphates are maintained. ${ }^{31}$ Quick metabolic reprogramming to anaerobic metabolism is therefore of critical importance to the heart's survival of an acute ischaemic insult. ${ }^{32}$ The Hif- $1 \alpha^{\text {tg }}$ hearts demonstrated signs of energy metabolism remodelling, with a shift towards glucose consumption. The ratio of ATP synthesis compared with 


\section{Original Publications}

A

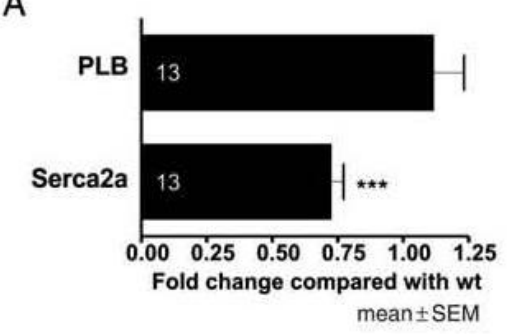

B

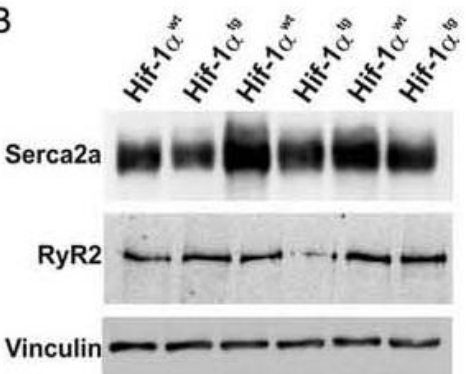

C
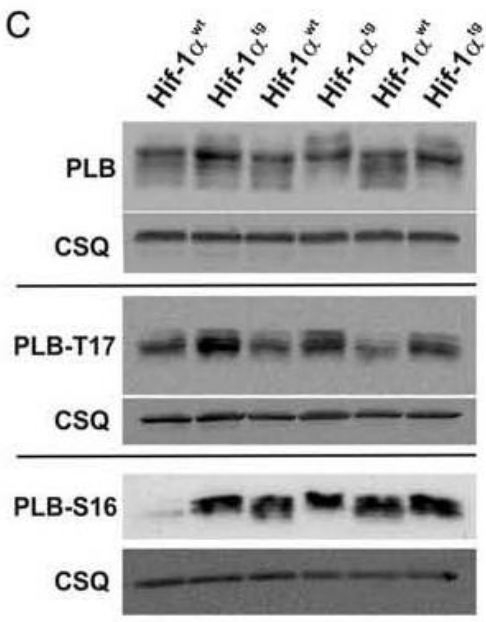

D

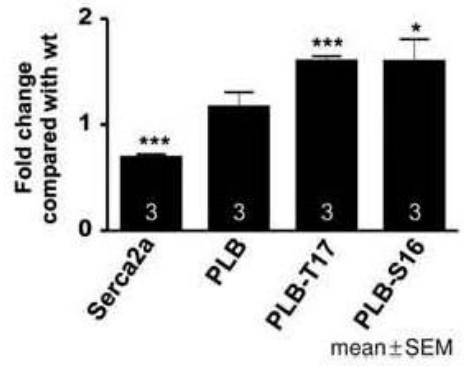

E

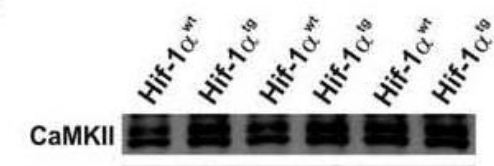

Vinculin $-\infty-\infty-$

pCaMKII - - . - -

Vinculin $\rightarrow-\rightarrow-\rightarrow$

F

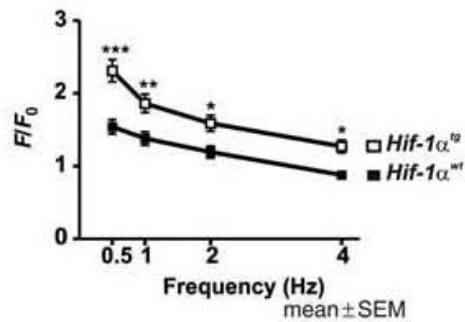

G

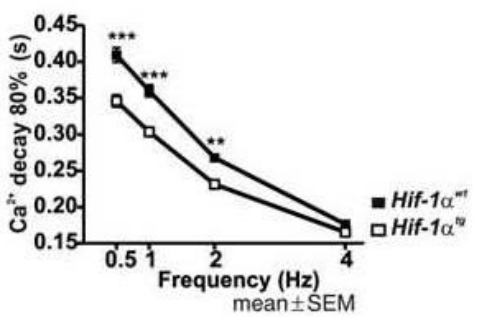

$\mathrm{H}$
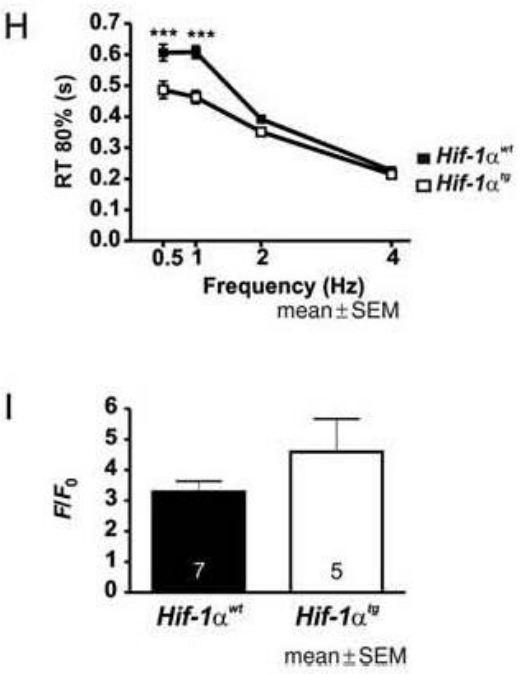

Figure 4 Changes in calcium handling in cardiomyocytes of $H i f f \alpha^{\text {tg }}$ mice. (A) RNA expression of SERCA2a and phospholamban (PLB) in left ventricles of 8 -week-old $H_{i f-1} \alpha^{\text {wt }}$ and $H_{i f-} 1 \alpha^{\text {tg }}$ mice was determined in quantitative real-time RT-PCR analysis $(* * * P<0.001)$. (B) SERCA2a protein levels were decreased and RyR2 protein levels unchanged in the left ventricles of Hif- $1 \alpha^{\text {tg }}$ mice compared with Hif- $1 \alpha^{\text {wt }}$ mice, as determined by western blot analysis. (C) Protein expression of calcium handling proteins was detected by western blot analysis of protein extracts from left ventricles of $H_{i f} 1 \alpha^{\text {wt }}$ and $H_{i f f} 1 \alpha^{\text {tg }}$ mice. (D) Quantification of SERCA2a, PLB, PLB T-17 and PLB-S16 protein levels as shown in A, B and C was determined by western blot analysis. (E) CaMKIl and phosphorylated CaMKIl protein levels were determined by western blot analysis. $(F-1) \mathrm{Ca}^{2+}$ homeostasis in isolated $\mathrm{Hif}_{\mathrm{f}} 1 \alpha^{\mathrm{wt}}$ and $\mathrm{Hif}-1 \alpha^{\text {tg }}$ cardiomyocytes. Cells were stimulated at $0.5-4 \mathrm{~Hz}$ and analysed for $\mathrm{Ca}^{2+}$ transient amplitude $\left(\mathrm{F} ;{ }^{* * P}<0.01\right.$, ${ }^{* * * P}<0.001$; data represent mean values \pm SEM), RT $80 \%$ intracellular $\left[\mathrm{Ca}^{2+}\right]$ decline $(G ; * * p<0.001$; data represent mean values \pm SEM), and $\mathrm{RT} 80 \%$ twitch relaxation $(H ; * * P<0.01, * * * p<0.001)$. (I) The sarcoplasmic reticulum $\mathrm{Ca}^{2+}$ content is unaltered in cardiomyocytes from Hif $1 \alpha^{\text {to }}$ mice measured by caffeine-induced $\mathrm{Ca}^{2+}$ transients. 


\section{Original Publications}

A
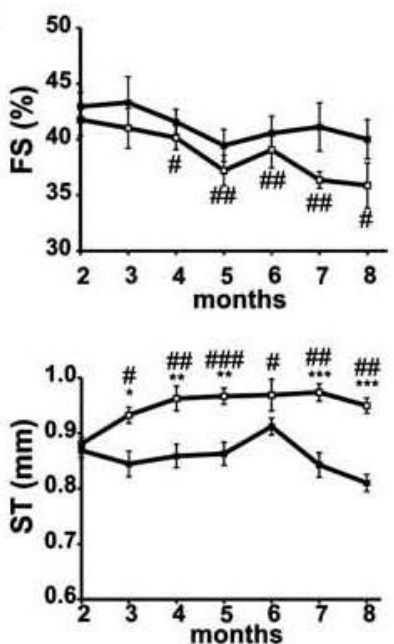

B

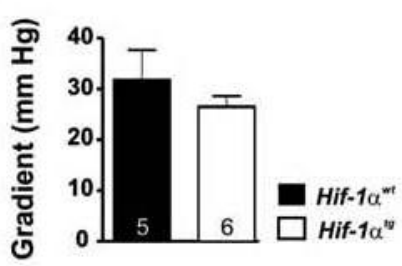

E

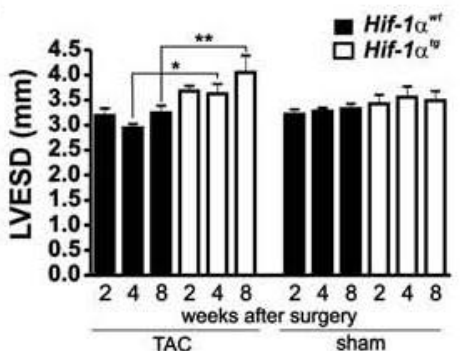

$\mathrm{H}$

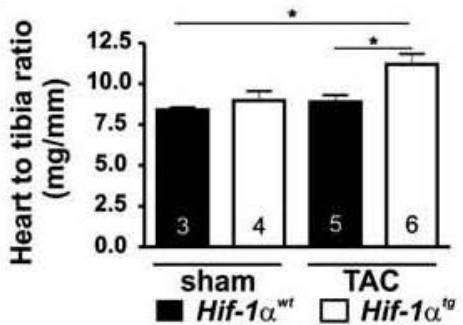

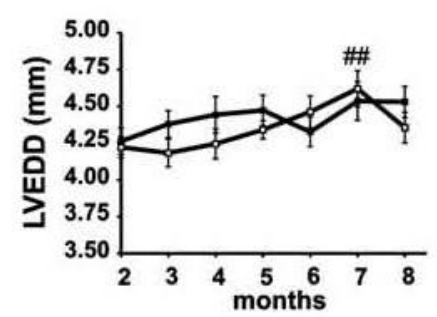

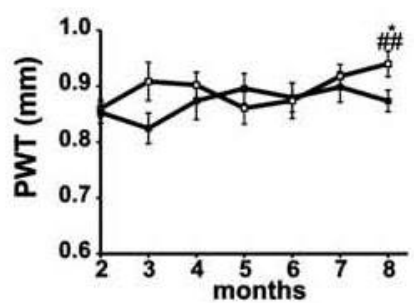

C

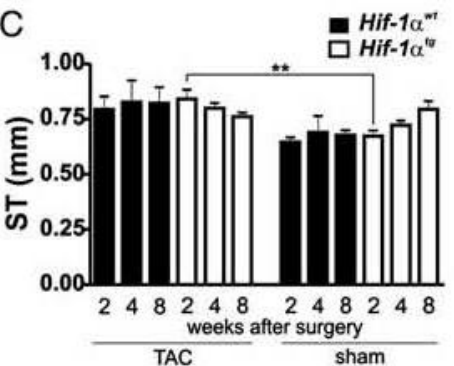

F

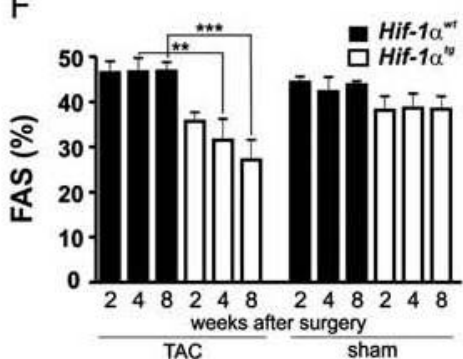

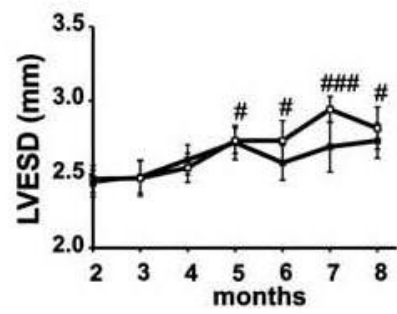

- Hif- $1 \alpha^{m}, n=8$ $\square H i f-1 \alpha^{t 0}, n=8$

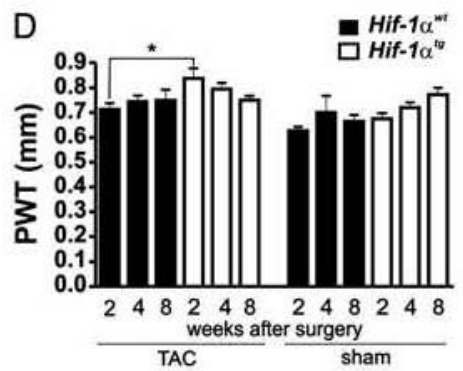

G

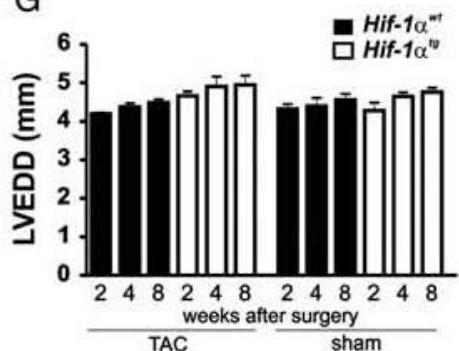

Figure 5 Hif-1 $\alpha^{\text {tg }}$ mice develop signs of heart failure with ageing and after transverse aortic constriction. (A) Echocardiographic measurements were performed serially on a cohort of male Hif- $1 \alpha^{\text {wt }}(n=8)$ and Hif $-1 \alpha^{\text {tg }}$ mice $(n=8)$ between the age of 2 and 8 months. Fractional shortening (FS), septum thickness (ST), posterior wall thickness (PWT), left ventricular end-diastolic diameter (LVEDD), and left ventricular end-systolic diameter (LVESD) were analysed $\left({ }^{*} P<0.05,{ }^{* *} P<0.01\right.$, ${ }^{* * * P}<0.001$, Hif- $1 \alpha^{\text {tg }}$ mice vs. Hif- $1 \alpha^{\text {wt }}$ control mice; ${ }^{\#} P<0.05,{ }^{\# \#} P<0.01$, ${ }^{\# \# p}<0.001$, aged Hiff $1 \alpha^{\text {tg }}$ mice vs. 2 -month-old Hif-1 $\alpha^{\text {tg }}$ mice). Sustained pressure overload was induced in female Hif- $1 \alpha^{\text {wt }}$ and Hif- $1 \alpha^{\text {tg }}$ mice by TAC [TAC treatment, Hif- $1 \alpha^{\text {wt }}(n=5)$ and Hif- $1 \alpha^{\text {tg }}(n=6)$; sham treatment, Hif- $1 \alpha^{\text {wt }}(n=3)$ and Hif- $\left.1 \alpha^{\text {tg }}(n=4)\right]$. (B) The TAC-induced pressure gradient was measured 1 day after surgery. Numbers in the bars indicate the number of animals in each group. ST (C), PWT (D), LVESD (E), fractional area shortening (FAS; $F$ ), and LVEDD $(G)$ were analysed 2, 4, and 8 weeks after TAC by echocardiography. $(H)$ The heart weight-to-tibia length ratios were determined 8 weeks after TAC $(* P<0.05)$. 


\section{Original Publications}

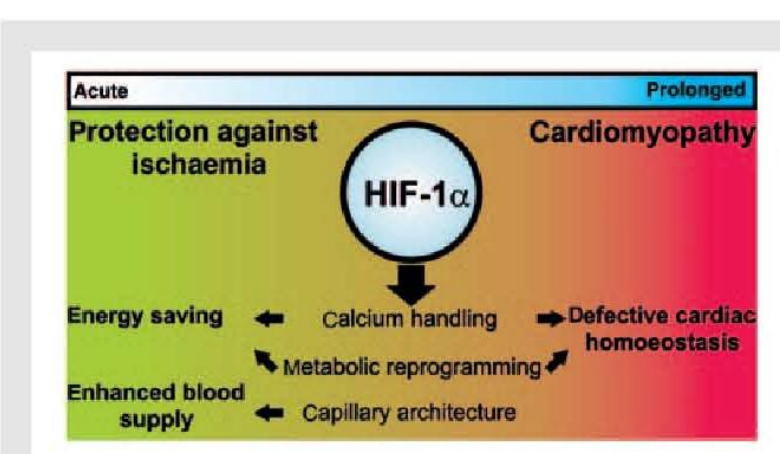

Figure 6 Scheme indicating possible mechanisms involved in protective and maladaptive effects of cardiac-specific HIF-1 $\alpha$ up-regulation. For details see Discussion.

the spent $\mathrm{O}_{2}$ is lower when glucose vs. fatty acids is consumed. This mechanism is used by the heart in the case of severe ischaemia and, based on our results demonstrated here, is transcriptionally controlled at least in part by HIF-1. On the molar basis over the long term, however, much more ATP is produced from fatty acid oxidation than from anaerobic glucose utilization. ${ }^{33}$ In uncompensated hypertrophy and in other forms of heart failure as a consequence of high energy demand, a sole increase in glucose uptake and utilization is therefore not sufficient to compensate for the overall energy demand. ${ }^{34}$ Thus, the HIF-1 $\alpha$ overexpressing heart seems to be well prepared to face an acute ischaemic insult via expression of anaerobic metabolism-related genes and adapting calcium handling. In line with our results, a previous study has shown that constitutive HIF-1 $\alpha$ overexpression protects the heart from diabetic-induced glucose metabolism impairment, partly by up-regulation of Glut- $1 .^{35}$ The metabolic preconditioning in HIF-1 $\alpha$ transgenic mice, however, seems not to be sufficient for the adaptation towards increased mechanical load, because energy supply may be a limiting factor in this hypertrophy-inducing and energy-wasting setting.

In summary, the cardiac stabilization of HIF-1 $\alpha$ results in tissue protection in the case of myocardial infarction. ${ }^{14}$ HIF-mediated pathways involved may include changes in vascular tone, energy metabolism, and calcium handling (Figure 6). According to our results, long-term activation of HIF-1 and its associated adaptive pathways is detrimental. Although short-term activation of HIF-1 is beneficial and may be used in therapeutic strategies, prolonged activation of HIF-1 over time drives the development of cardiomyopathy.

\section{Supplementary material}

Supplementary material is available at Cardiovascular Research online.

\section{Acknowledgements}

We would like to acknowledge the technical support of Annette Hillemann, Anika Hunold, Felicia Steuer, and Thomas Sowa. Mareike Lehnhoff, Maren König, and Daniel Bukala were of valuable help in PET measurements. The radiopharmacy group, Gerald Reischl, Denis Lamparter, and Walter Ehrlichmann, of the Department of Preclinical Imaging and Radiopharmacy at the University Hospital of Tübingen, is acknowledged for radiotracer production. We also thank Samantha Whitman (University of Arizona, Tucson, AZ, USA) for her help preparing this manuscript.

Conflict of interest: none declared.

\section{Funding}

This work was supported by grants from the Else Kröner-Fresenius-Stiftung (P25/10//A12/10 to A.Z); and the Deutsche Forschungsgemeinschaft (Ka1269/11-1 to D.M.K.).

\section{References}

1. Mudd JO, Kass DA. Tackling heart failure in the twenty-first century. Nature 2008;451 919-928.

2. Giordano FJ. Oxygen, oxidative stress, hypoxia, and heart failure. f Gin invest 2005; 115:500-508.

3. Majmundar AJ, Wong W], Simon MC. Hypoxia-inducible factors and the response to hypoxic stress. Mol Cell 2010;40:294-309.

4. Jewell UR, Kvietikova I, Scheid A, Bauer C, Wenger RH, Gassmann M. Induction of HIF-1 $\alpha$ in response to hypoxia is instantaneous. FASEB / 2001;15:1312-1314.

5. Ivan M, Kondo K, Yang H, Kim W, Valiando J, Ohh M et at. HIFo targeted for VHL-mediated destruction by proline hydroxylation: implications for $\mathrm{O}_{2}$ sensing. Science 2001;292:464-468.

6. Jaakkola P, Mole DR, Tian YM, Wilson MI, Gielbert J, Gaskell Sj et al. Targeting of $\mathrm{HIF}-\alpha$ to the von Hippel-Lindau ubiquitylation complex by $\mathrm{O}_{2}$-regulated prolyl hydroxylation. Science 2001;292:468-472.

7. Hon WC, Wilson MI, Harlos K, Claridge TD, Schofield C], Pugh CW et al. Structural basis for the recognition of hydroxyproline in HIF-1 $\alpha$ by pVHL. Nature 2002;417: 975-978.

8. Min JH, Yang H, Ivan M, Gertler F, Kaelin WG Jr, Pavletich NP. Structure of an HIF-1 $\alpha$-pVHL complex: hydroxyproline recognition in signaling. Science 2002;296: $1886-1889$.

9. Wenger RH, Stiehl DP, Camenisch G. Integration of oxygen signaling at the consensus HRE. Sci STKE 2005;2005:re12.

10. Eckle T, Kohler D, Lehmann R, El Kasmi K, Eltzschig HK. Hypoxia-inducible factor- 1 is central to cardioprotection: a new paradigm for ischemic preconditioning. Circulation 2008;118:166-175.

11. Hölscher M, Silter M, Krull S, von Ahlen M, Hesse A, Schwartz $P$ et at. Cardiomyocyte-specific prolyl-4-hydroxylase domain 2 knock out protects from acute myocardial ischemic injury. J Biol Chem 2011;286:11185-11194.

12. Hyvarinen J, Hassinen IE, Sormunen R, Maki JM, Kivirikko KI, Koivunen P et al. Hearts of hypoxia-inducible factor prolyl 4-hydroxylase-2 hypomorphic mice show protec tion against acute ischemia-reperfusion injury. f Biol Chem 2010;285:13646-13657.

13. Myllyharju J. HIF prolyl 4-hydroxylases and their potential as drug targets. Curr Pharm Des 2009;15:3878-3885.

14. Kido M, Du L, Sullivan CC, Li X, Deutsch R, Jamieson SW et al. Hypoxia-inducible factor $1 \alpha$ reduces infarction and attenuates progression of cardiac dysfunction after myocardial infarction in the mouse. I Am Coll Cardiol 2005;46:2116-2124.

15. Silter M, Kögler H, Zieseniss A, Wilting J, Schäfer K, Toischer K et al. Impaired $\mathrm{Ca}^{2+}$ handling in HIF- $1 \alpha^{+l}$ mice as a consequence of pressure overload. Pflugers Arch 2010;459:569-577.

16. Zieseniss A, Schroeder U, Buchmeier S, Schoenenberger CA, van den Heuvel J, Jackusch BM et al. Raver 1 is an integral component of muscle contractile elements. Cell Tissue Res 2007;327:583-594.

17. El-Armouche A, Ouchi N, Tanaka K, Doros G, Wittköpper K, Schulze T et at. Follistatin-like 1 in chronic systolic heart failure: a marker of left ventricular remodeling. Circ Heart Fait 2011;4:621-627.

18. Lee SH, Wolf PL, Escudero R, Deutsch R, Jamieson SW, Thistlethwaite PA. Early expression of angiogenesis factors in acute myocardial ischemia and infarction. $N$ Engl f Med 2000;342:626-633.

19. Krishnan J, Suter M, Windak R, Krebs T, Felley A, Montessuit C et al. Activation of a HIF-1 $\alpha$-PPAR $y$ axis underlies the integration of glycolytic and lipid anabolic pathways in pathologic cardiac hypertrophy. Cell Metab 2009;9:512-524.

20. Pescador N, Villar D, Cifuentes D, Garcia-Rocha M, Ortiz-Barahona A, Vazquez S et at. Hypoxia promotes glycogen accumulation through hypoxia inducible factor (HIF)-mediated induction of glycogen synthase 1. PLoS One 2010;5:e9644.

21. Ronkainen VP, Skoumal R, Tavi P. Hypoxia and HIF-1 suppress SERCA2a expression in embryonic cardiac myocytes through two interdependent hypoxia response elements. J Mol Cell Cardiol 2011;50:1008-1016.

22. Li L, Chu G, Kranias EG, Bers DM. Cardiac myocyte calcium transport in phospholamban knockout mouse: relaxation and endogenous CaMKII effects. Am J Physio 1998;274: H1335-H1347.

23. Knuuti J, Tuunanen $\mathrm{H}$. Metabolic imaging in myocardial ischemia and heart failure. Qf Nucl Med Mol Imaging 2010;54:168-176. 


\section{Original Publications}

24. Hasenfuss G, Schillinger W, Lehnart SE, Preuss M, Pieske B, Maier LS et at. Relationship between $\mathrm{Na}^{+} \cdot \mathrm{Ca}^{2+}$-exchanger protein levels and diastolic function of failing human myocardium. Groulation 1999:99:641-648.

25. Taegtmeyer $\mathrm{H}$, Dilsizian V. Imaging myocardial metabolism and ischemic memory. Not Cin Pract Cardiovasc Med 2008;5:S42-S48

26. Bao W, Qin P, Needle S, Erickson-Miller CL, Duffy KJ, Ariazi JL et al. Chronic inhibition of hypoxia-inducible factor (HIF) prolyl 4-hydroxylase improves ventricular performance, remodeling and vascularity following myocardial infarction in the rat J Cardiovasc Pharmacol 2010;56:147-155.

27. Huang M, Chan DA, jia F, Xie X, Li Z, Hoyt G et of. Short hairpin RNA interference therapy for ischemic heart disease. Circulation 2008;118:S226-S233.

28. Lei L, Mason S, Liu D, Huang Y, Marks C. Hickey R et al. Hypoxia-inducible factordependent degeneration, failure, and malignant transformation of the heart in the absence of the von Hippel-Lindau protein. Mol Cell Biot 2008;28:3790-3803.

29. Bekeredjian R, Walton CB, MacCannell KA, Ecker ], Kruse F, Outten JT et ol. Conditional HIF-1 $\alpha$ expression produces a reversible cardiomyopathy. PLOS One 2010;5: e11693.
30. Moslehi J, Minamishima YA, Shi J, Neuberg D, Charytan DM, Padera RF et at. Loss of hypoxia-inducible factor prolyl hydroxylase activity in cardiomyocytes phenocopies ischemic cardiomyopathy. Circulation 2010;122:1004-1016.

31. Taegtmeyer $H$, Wilson CR, Razeghi P, Sharma S. Metabolic energetics and genetics in the heart. Ann N Y Acad Só 2005;1047:208-218.

32. Jennings RB, Reimer KA. The cell biology of acute myocardial ischemia. Annu Rev Med 1991:42:225-246.

33. Ingwall JS. Energy metabolism in heart failure and remodelling. Cardiovasc Res 2009;81 $412-419$.

34. Neglia D, De Caterina A, Marraccini P, Natali A, Ciardetti M, Vecoli C et of. Impaired myocardial metabolic reserve and substrate selection flexibility during stress in patients with idiopathic dilated cardiomyopathy. Am J Physiol Heart Grc Physiol 2007; 293: $\mathrm{H} 3270-\mathrm{H} 3278$

35. Xue W, Cai L, Tan Y, Thistlethwaite P, Kang Y], Li X et af. Cardiac-specific overexpression of HIF- $1 \alpha$ prevents deterioration of glycolytic pathway and cardiac remodeling in streptozotocin-induced diabetic mice. Am \& Pathol 2010;177: 97-105. 


\section{Original Publications}

\section{Supplemental figures}

Fig.1
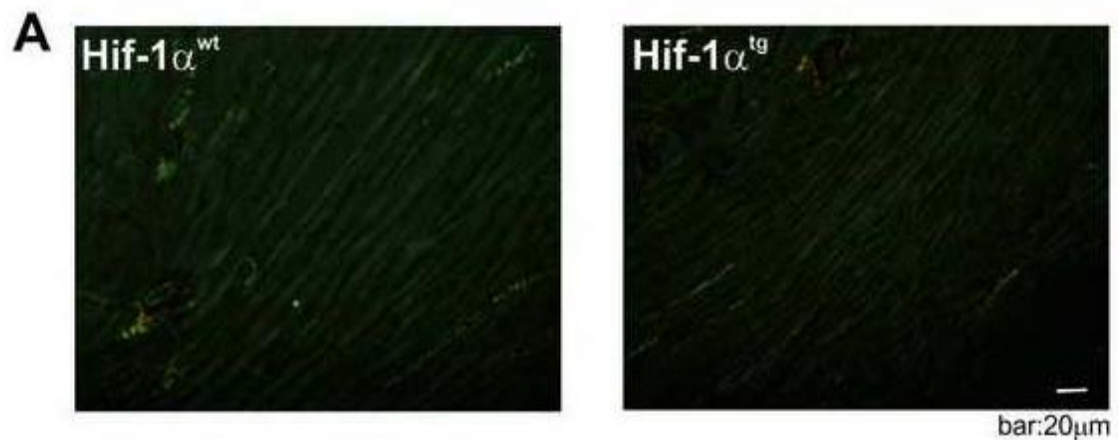

Fig.2

A

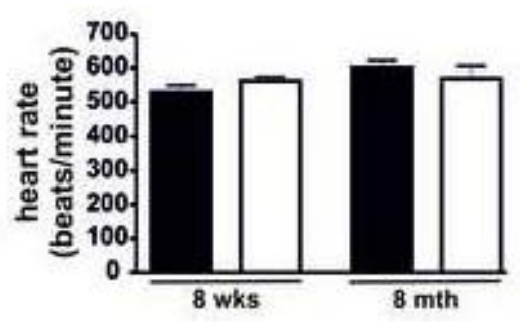

Hif $-1 \alpha^{* 4}, n=4$ (8wks), $n=8$ (8mth) $\square$ Hif- $1 \alpha^{*}, n=5$ (8wks), $n=8$ (8mth)

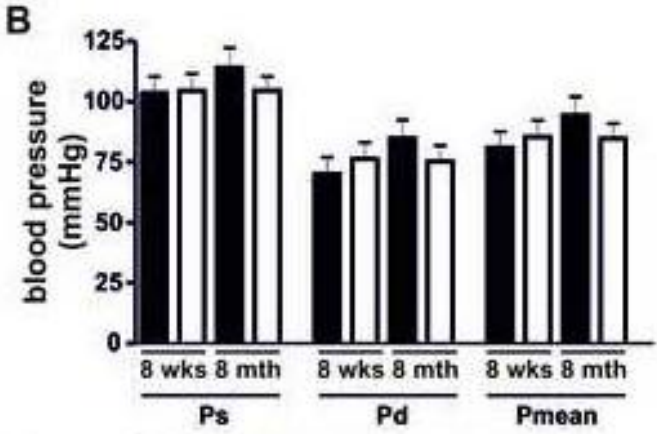

Hif-1a $\alpha^{-1}, n=4$ (8wks), $n=8$ (8mth)

$\square$ Hif- $1 \alpha^{*}, n=5$ (8wks), $n=8$ (8mth)
C

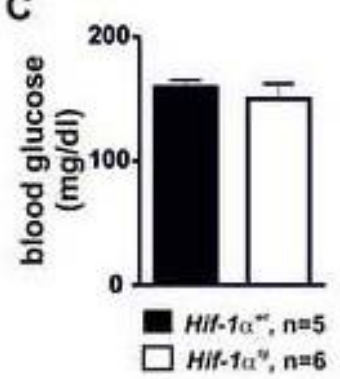

D

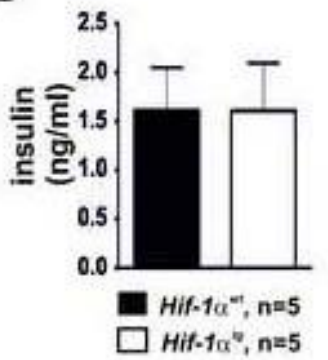

E

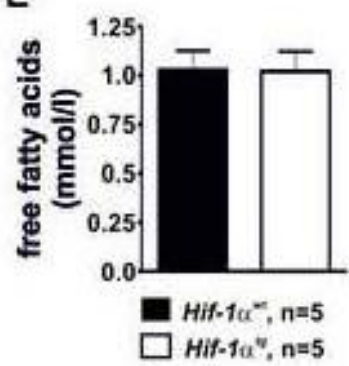




\section{Original Publications}

Fig.3

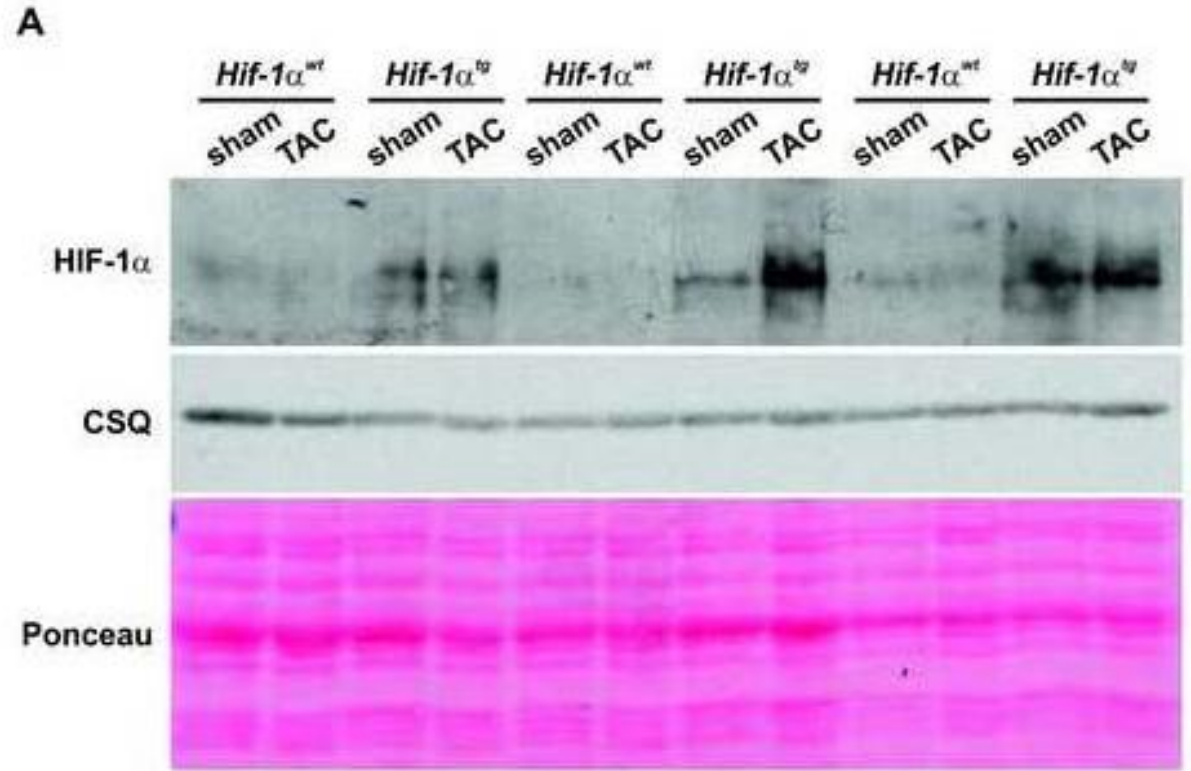

B

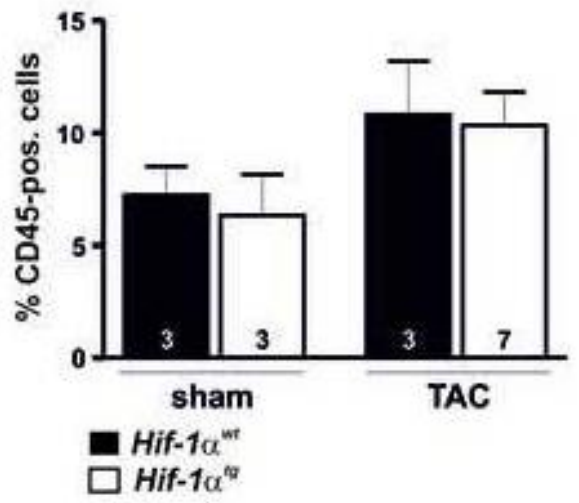

Supp. Fig. 1. Histological staining for collagen (Sirius Red) on Hif- $1 \alpha^{w t}$ and Hif- $1 \alpha^{t g}$ hearts.

Supp. Fig. 2. Heart rate (A), blood pressure (B) analyzed in 8 weeks and 8 month old mice, blood glucose (C), insulin (D) and free fatty acids (E) do not differ between Hif-1 $\alpha^{\text {wt }}$ and Hif$1 \alpha^{\text {tg }}$ hearts.

Supp. Fig. 3. (A) HIF-1 $\alpha$ protein was detected by Western blot analysis of protein extracts from left ventricles of Hif- $1 \alpha^{\text {wt }}$ and Hif- $1 \alpha^{\text {tg }}$ mice 8 weeks after sham- and TAC-treatment. (B) Myocardial inflammation was analyzed in sham and TAC treated Hif- $1 \alpha^{\text {wt }}$ and Hif- $1 \alpha^{\text {tg }}$ hearts by CD45 flow cytometry. 


\section{Original Publications}

\section{Supplemental Methods}

\section{Primer sequences}

mS12 forward, 5'-GAAGCTGCCAAGGCCTTAGA-3' and mS12 reverse, 5'AACTGCAACCAACCACCTTC-3'; HIF-1 $\alpha$ forward, 5'-TGCATCTCCATCTCCTACCC -3 ' and HIF-1 $\alpha$ reverse, 5'-GTAGCTGCATGATCGTCTGG-3'; Glut1 forward, 5'-TGGCCTTGCTGGAACGGCTG-3' and Glut1 reverse, 5'-TCCTTGGGCTGCAGGGAGCA-3'; phosphofruktokinase 1 (Pfkl) forward, 5'-ACGAGgCCATCCAGCTCCGT-3' and Pfkl reverse, 5'-TGGGGCTTGGGCAGTGTCCT-3'; carbonic anhydrase IX (CA IX) forward, 5'-GGGGGTCATCTGGACTGTGTT-3' and CA IX reverse, 5'-CCCACTTCTGTGCCTGTGCT-3'; Glucan (1,4-alpha) branching enzyme (GBE1) forward, 5'GCTTTCAGCTCCACAGGCCTACG -3' and GBE1 reverse, 5'-TGAGGGGGATCAGCGGCAACA-3'; Glycogen synthase 1 (Gys1) forward, 5'- CCCTGGGACACCCGCTAACTCTAC-3' and Gys1 reverse, 5'- CCCCTGTCACCTTCGCCTTCG -3'; adrenomedullin (Adm) forward, 5'TGGCCCCCTACAAGCCAGCAAT-3' and adrenomedullin reverse, 5'GCCAACGGGATACTCGCCCG-3'; apelin forward, 5'-TGCAGTTTGTGGAGTGCCACTG-3' and apelin reverse, 5'-GCACCGGGAGGCACT-3'; VEGFa forward, 5'CACGACAGAAGGAGAGCAGAAGT-3' and VEGFa reverse, 5'- TTCGCTGGTAGACATCCATGAA-3'; angiopoietin (Ang)-1 forward, 5'-CGCTCTCATGCTAACAGGAGGTTGG-3' and Ang-1 reverse, 5' GCATTCTCTGGACCCAAGTGGCG-3'; Ang-2 forward, 5'-CACCCAACTCCAAGAGCTCGG-3' and Ang-2 reverse, 5'-CACGTAGCGGTGCTGACCGG-3'; Phospholamban (PLB) forward 5'CCGAAGCCAAGACAGAAGCAGGTG-3' and PLB reverse, 5'-CCCATGACGGA GTGCTCGGC-3'; SERCA2a forward, 5'-AGATGGTCCTGGCAGATGAC-3' and SERCA2a reverse, 5'CCAGGTCTGGAGGATTGAAC -3', PPAR $\alpha$ forward 5'-CACGGCGTGGTGC ATTTGGG-3' and PPAR $\alpha$ reverse 5'- TGACAGAGCCCTCGGAGCCC-3'; PPARY forward 5'ACGGGGTCTCGGTTGAGGGG-3`and PPAR $p$ reverse 5' -TCCGCCCAAACCTGATGGCATT-3'; CD36 forward 5'-AATTCCCTTGGCAACCAACCACA-3' and CD36 reverse 5'GTGGCCCGGTTCTACTAATTCATGA-3'; iNOS forward 5'-CCTCACTGGGACAGCACAGAATGT-3' and iNOS reverse 5 '-CCGATGCAGCGAGGGGCATT-3`.

\section{Antibodies used for Western blots}

Membranes were probed with anti-HIF-1 $\alpha$ (Novus), anti-Vinculin (Sigma), anti-Calsequestrin (Affinity Bioreagents), anti-Serca2a (Dianova), anti-RyR2 (Acris), anti-CaMKII (a gift from D. M. Bers, University of California, Davis, Calif, to L.S. Maier), anti-pospho-CaMKII (Thermo 


\section{Original Publications}

Scientific), anti-Phospholamban (Badrilla), anti-Phospho-Thr17 (Badrilla) anti-Phospho-S16 (Badrilla) followed by a goat HRP (horseradish peroxidase)-labelled anti-mouse or anti-rabbit antibody (Santa Cruz Biotechnology).

\section{TAC surgery}

Pressure overload was induced by TAC in 5-7 week old female mice. Mice were anesthetized by $1.5 \%$ isoflurane inhalation. Pain management of the mice comprised treatment with buprenorphine (0.06 mg/kg body weight i.p. before surgergy) and $1.33 \mathrm{mg} / \mathrm{ml}$ metamizole (for 7 days in the drinking water). Once anesthesia was administered the anesthetic plane was assessed by the reaction to pinching the toe, determining the respiration rate and close clinical supervision. The aorta was constricted with polyviolene non-absorbable braided nylon strings (5-0 USP) using blunted 25-gauge needles as placeholders that were removed after ligation.

\section{Echocardiography}

For transthoracic echocardiography after TAC a Vevo2100 (VisualSonics, Toronto, Canada) system with a $30 \mathrm{MHz}$ center frequency transducer was used. Two-dimensional cine loops with frame rates of 200 frames/s of a long axis view and a short-axis with at mid-level of the papillary muscles as well as M-mode loops of the short axis view were recorded. Thickness of the septum (ST), the posterior myocardial wall (PWT), and the area of the left ventricular cavity were measured in systole and diastole from the short-axis view. Fractional area shortening was calculated: \% FAS = (LV end-diastolic area - LV end-systolic area)/ (LV enddiastolic areas $\times 100)$.

\section{Mouse heart rate and blood pressure measurements}

Heart rate and blood pressure were measured in 8 week and 8 month old mice using a CODA non-invasive blood pressure system (Kent Scientific Corporation, Connecticut, USA).

\section{Radiotracer production}

Fluorine-18 was produced as $\left[{ }^{18}\right.$ F]fluoride at the PETtrace cyclotron (General Electric Healthcare, Uppsala, Sweden) using the $180(p, n) 18 \mathrm{~F}$ nuclear reaction. [ $\left.{ }^{18} \mathrm{~F}\right] \mathrm{Fluoro}-$ 2deoxyglucose $\left(\left[{ }^{18} \mathrm{~F}\right] \mathrm{FDG}\right)$ was synthesized in a FDG MicroLab module (GE Healthcare, 


\section{Original Publications}

Münster, Germany) as published ${ }^{1}$. Radiochemical purity was $>95 \%$. [ $\left.{ }^{18 F}\right] \mathrm{FTHA}$ was synthesized using the method from DeGrado ${ }^{2}$ with modifications on a modified TRACERlab FXF-N PET tracer Synthesizer (GE Healthcare, Münster, Germany). Briefly, $2 \mu \mathrm{L}$ of the precursor benzyl-14-(R,S)-tosyloxy-6-thiaheptadecanoate (ABX, Germany) in $1 \mathrm{~mL}$ of acetonitrile were reacted with a mixture of aceotropically dried $\left[{ }^{18} \mathrm{~F}\right]$ fluoride, $15 \mathrm{mg}$ of Kryptofix 2.2.2. and $3.5 \mathrm{mg} \mathrm{K}_{2} \mathrm{CO}_{3}$ at $110^{\circ} \mathrm{C}$ for $5 \mathrm{~min}$. After hydrolysis with $350 \mu \mathrm{L}$ of $0.14 \mathrm{~N}$ $\mathrm{KOH}\left(110{ }^{\circ} \mathrm{C}, 5 \mathrm{~min}\right) 0.3 \mathrm{~mL}$ of $6.5 \%$ sulfuric acid were added for neutralization. The product was purified using HPLC chromatography (Supelcosil ABZ+; $10 \times 250$ mm; $\mathrm{H}_{2} \mathrm{O} / \mathrm{MeOH} \mathrm{80/20}$ with $1 \% \mathrm{H}_{3} \mathrm{PO}_{4} ; 5 \mathrm{ml} / \mathrm{min}$; detection: UV $216 \mathrm{~nm}$ and Nal(TI)). The product was obtained in uncorrected yields of $15 \pm 5 \%(n=13)$, corresponding to $9.3 \pm 3.3 \mathrm{GBq}$ of isolated $\left[{ }^{18} \mathrm{~F}\right] \mathrm{FTHA}$, after irradiations using 35 to $60 \mu \mathrm{A}$ for 40 to $60 \mathrm{~min}$. Radiochemical purity as determined by TLC was $>90 \%$. Specific activities were $>50 \mathrm{GBq} / \mu \mathrm{mol}$ at end of synthesis.

1. Hamacher K, Coenen HH, Stocklin G. Efficient stereospecific synthesis of no-carrieradded 2-[ $\left.{ }^{18} \mathrm{~F}\right]$-fluoro-2-deoxy-d-glucose using aminopolyether supported nucleophilic substitution. J Nucl Med 1986;27:235-238.

2. DeGrado TR, Coenen HH, Stocklin G. 14(r,s)-[ ${ }^{18}$ F]fluoro-6-thia-heptadecanoic acid (ftha): Evaluation in mouse of a new probe of myocardial utilization of long chain fatty acids. J Nucl Med 1991;32:1888-1896.

\section{PET analysis}

Before each scan day the mice were fasted for at least $14 \mathrm{hr}$. For anesthesia $1.5 \%$ isoflurane was evaporated in medical oxygen using a dedicated vaporizer for rodents (Vetland, Lousiville, KY, USA). After the tail vein i.v. injection of $\left[{ }^{18} \mathrm{~F}\right] \mathrm{FTHA}(13 \pm 1.03 \mathrm{MBq})$ or $\left[{ }^{18} \mathrm{~F}\right] \mathrm{FDG}$ $(13 \pm 0.3 \mathrm{MBq})$ and a $55 \mathrm{~min}$ uptake time under anesthesia, a $15 \mathrm{~min}$ emission scan was obtained. This PET measurement was followed by 13 min transmission scan based on a rotating ${ }^{57}$ Co source for photon attenuation correction. The PET images were reconstructed using an ordered subset expectation maximization (OSEM) 2D algorithm with attenuation correction (matrix: $128 \times 128$; voxel size: $0.776 \times 0.776 \times 0.796 \mathrm{~mm}^{3}$ ). For image analysis regions of interests (ROIs) were placed on 3 consecutive slices of the middle left myocardium in short axis view to avoid the spillover effect from liver to the heart apex region. The counts of left ventricle cavity were subtracted. The myocardial $\left[{ }^{18} \mathrm{~F}\right] \mathrm{FTHA}$ and $\left[{ }^{18} \mathrm{~F}\right] \mathrm{FDG}$ uptake was reported as standardized uptake value (SUV, SUV = tissue radioactivity concentration 


\section{Original Publications}

$[\mathrm{Bq} / \mathrm{ml}] /$ (injection dose $[\mathrm{Bq}] /$ body weight $[\mathrm{g}]$ )). One mouse of the transgenic group was excluded from $\left[{ }^{18}\right.$ F]FDG SUV evaluation because it was not anesthetized properly during the PET scan.

\section{Sirius Red Polarization Method for Collagen Staining}

Interstitial collagen was detected by Sirius red polarization microscopy. Paraffin sections were stained with $0.1 \%$ Sirius red in saturated picric acid for $1 \mathrm{~h}$. Sections were rinsed with acidified water for 1 minute twice and then dehydrated with $100 \%$ ethanol, sections were coverslipped. The stained sections were observed under polarized light and photographed with the same exposure time for each section.

\section{Cryosections and immunofluorescence labelling}

Hearts were sectioned $(5-10 \mu \mathrm{m})$ in a cryostat, mounted on glass slides and dried for at least 30 min. After being washed with PBS, the sections were briefly incubated with $1 \%$ Triton X100 and then fixed with $4 \%$ formaldehyde and permeabilized with $0.2 \%$ Triton $X-100$. Nonspecific binding of antibodies was blocked by incubation with $1 \%$ bovine serum albumin for 1 h. The sections were then incubated with the polyclonal anti-CD31 antibodies (Dianova, DIA 310) and anti-vinculin antibodies (Sigma, hVin-1) at room temperature for $1 \mathrm{~h}$, washed three times in PBS and then incubated with anti-mouse TR (diluted 1:200; SantaCruz) and anti-rat FITC (1:200, SantaCruz). Samples were counterstained for DNA (Hoechst). Finally, samples were extensively washed in PBS, mounted in Mowiol, and examined by fluorescence microscopy (Axio Observer D1, Carl Zeiss, Göttingen, Germany). Capillary area was determined by analyzing CD31 positive pixels using ImageJ (NIH Bethesda, MD). In addition, numbers of capillaries were counted per view of field.

Isolation of cardiomyocytes, cardiomyocyte shortening and intracellular $\mathrm{Ca}^{2+}$ measurements

Isolated hearts were mounted on a Langendorff-perfusion apparatus driven by gravity and perfused with nominally $\mathrm{Ca}^{2+}$-free Tyrode's solution containing (in $\mathrm{mM}$ ) $\mathrm{NaCl} 115, \mathrm{KCl} 4.7$, $\mathrm{KH}_{2} \mathrm{PO}_{4}$ 0.6, $\mathrm{Na}_{2} \mathrm{HPO}_{4}$ 0.32, $\mathrm{MgSO}_{4}$ 1.2, $\mathrm{NaHCO}_{3}$ 12, $\mathrm{KHCO} 3$ 10, HEPES 10, taurine 30, 2,3butanedionemonoxime 10 , glucose $5.5(\mathrm{pH} 7.46)$ for $2-4 \mathrm{~min}$ at $37^{\circ} \mathrm{C}$. Perfusion was then switched to the same solution containing Liberase blendzyme 1 (Roche) $0.25 \mathrm{mg} / \mathrm{ml}$ and 


\section{Original Publications}

Trypsin $0.14 \mathrm{mg} / \mathrm{ml}$ with perfusion continuing until the heart became flaccid (7-12 $\mathrm{min}$ ). Ventricular tissue was removed, dispersed, filtered and suspensions were rinsed several times. After $\mathrm{Ca}^{2+}$ reintroduction (stepwise increase to $0.8 \mathrm{mM}$ ), isolated myocytes were then plated onto superfusion chambers, with the glass bottoms treated with laminin to allow cell adhesion and used for immediate measurements. Myocytes were loaded with fluo-3 by incubation with $10 \mu \mathrm{M}$ of the acetoxymethyl ester (AM) form of the dye (Molecular Probes, Eugene, OR) for $15 \mathrm{~min}$ at room temperature in darkness. The dye was excited with a wavelength at $480 \pm 15 \mathrm{~nm}$ using a $75 \mathrm{~W}$ xenon arc lamp (Ushio, Japan) on the stage of a Nikon Eclipse TE200-U inverted microscope. Emitted fluorescence was measured using a photomultiplier (at 535 $\pm 20 \mathrm{~nm}$; lonOptix Corp., Milton, MA).

\section{Flow cytometry analysis of CD45 inflammatory cells}

The tissue was treated with $1.2 \mathrm{U} / \mathrm{mL}$ Dispasell (PAN), $150 \mathrm{U} / \mathrm{mL}$ Collagenase IV (Biochrom) and $2 \mathrm{mM} \mathrm{CaCl}_{2}$ in $\mathrm{PBS}$ for $45 \mathrm{~min}$ at $37^{\circ} \mathrm{C}$. Digestion was stopped using DMEM supplemented with 15\% FCS and remaining connective tissues and fibers were removed using $70 \mu \mathrm{m}$ and $40 \mu \mathrm{m}$ nylon cell strainers (BD). Cells were spun down at $400 \mathrm{~g}$ and $4^{\circ} \mathrm{C}$ and resuspended in erythrocyte lysis buffer containing $10 \mathrm{mM} \mathrm{KHCO}_{3}, 155 \mathrm{mM} \mathrm{NH}_{4} \mathrm{Cl}$ and 126 $\mu \mathrm{M}$ EDTA. Lysis was stopped after 5 min with DMEM + 15\% FCS and cells were again centrifuged at $400 \mathrm{~g}$ and $4^{\circ} \mathrm{C}$. Cells were resuspended in PBS and stained with PerCPCy5.5conjugated CD45 antibody or respective isotype controle (BD) and measured on a Becton Dickinson FACSCalibur. Cells were gated according to size (FCS-A) and granularity (SSC-A) as well as SSC-W to exclude cell debris and duplets. Using FACSdiva software percentage of CD45-positive cells within the gated population was analyzed. It should be noted that the analysis is suitable for detecting changes in the percentage of CD45 positive immune cells among different treatment groups but does not allow to quantify absolute CD45-positive cell numbers since disruption and digestion might release more small non-integrated cells (like leukocytes and endothelial cells) while dissociation of fibroblasts and the myocyte network can be less efficient. 


\section{Original Publications}

\section{Insulin measurements}

Insulin concentrations in serum of 8 month old mice were determined using the Ultra Sensitive Mouse Insulin ELISA kit (Crystal Chem, Inc.) according to manufacturer's instructions.

\section{Measurements of free fatty acids}

Free fatty acids in mouse serum of 8 month old animals were analyzed using assay kits from Wako (Osaka, Japan) and a clinical analyzer (Hitachi 917).

\section{Blood glucose level analysis}

$5 \mu \mathrm{l}$ whole blood was drawn from retrobulbar by a capillary when the mice were under $1.5 \%$ isoflurane anesthesia. After the blood was collected, the blood glucose level was measured immediately by a glucose analyzer (Hemocue, Ängelholm, Sweden). 


\section{Unpublished Data}

\section{Unpublished Data}

The following chapter contains additional data of the cardiomyocyte-specific PHD2 knockout mice $\left(c P h d 2^{--}\right)$that were not published at the submission date of this thesis and furthermore unpublished data of cardiomyocyte-specific PHD2/PHD3 double knock-out mice $\left(\right.$ cPhd $\left.2 / 3^{-/}\right)$.

\subsection{Detrimental consequences of a cardiomyocyte-specific knock-out of PHD2}

Marion Hölscher ${ }^{1}$, Yun Lin ${ }^{2}$, Bernd J. Pichler ${ }^{2}$, Ben Wielockx ${ }^{3}$, Georg Breier ${ }^{3}$, Dörthe M. Katschinski $^{1 *}$, Anke Zieseniss ${ }^{1 *}$ ( ${ }^{*}$ both authors contributed equally)

(1) From the Department of Cardiovascular Physiology, Universitätsmedizin Göttingen, Georg-August University Göttingen, (2) from the Department of Preclinical Imaging and Imaging Technology of the Werner Siemens-Foundation, University of Tübingen and (3) from the Department of Pathology, TU Dresden

\section{Introduction}

Cardiomyocyte-specific PHD2 knock-out $\left(\mathrm{cPhd}^{--}\right)$mice are protected from acute myocardial infarction as shown earlier by our group (Hölscher et al., 2011). However we did not analyze the long-term consequences of cardiomyocyte-specific PHD2 knock-out. Therefore, we followed a cohort of $c P h d 2^{-/}$and $w t$ littermates over a time period of eight months. $c P h d 2^{-/-}$ mice developed a hypertrophy with aging. Furthermore the metabolism of eight months old cPhd $2^{-}$mice was shifted towards glycolysis with an increased uptake of glucose and a diminished uptake of fatty acid as shown by PET analysis which was not detectable in eight week old mice.

\section{Material and methods}

Mice- Cardiomyocyte-specific PHD2 knock-out mice ( $c P h d 2^{-/-}$) (described in Hölscher et al., 2011) were further analyzed.

Echocardiography- Two-dimensional images and M-mode tracings were recorded from the parasternal long axis view at midpapillary level (Vevo 660 ${ }^{\text {тM }}$, VS-0 M-VE660 version 3.1, Visual Sonics). Posterior wall thickness (PWT) and fractional shortening (FS) were determined. FS was calculated as (systolic diameter-diastolic diameter/diastolic diameter $\mathrm{x}$ 100). FS was used as marker for cardiac contractile function.

Pet analysis- The PET analysis was done as described previously in Hölscher et al., 2012. 


\section{Unpublished Data}

\section{Results}

Cardiomyocyte-specific PHD2 knock-out animals develop cardiac hypertrophy with aging but without impairment of the heart function (fig. 1).

\section{Fig.1}
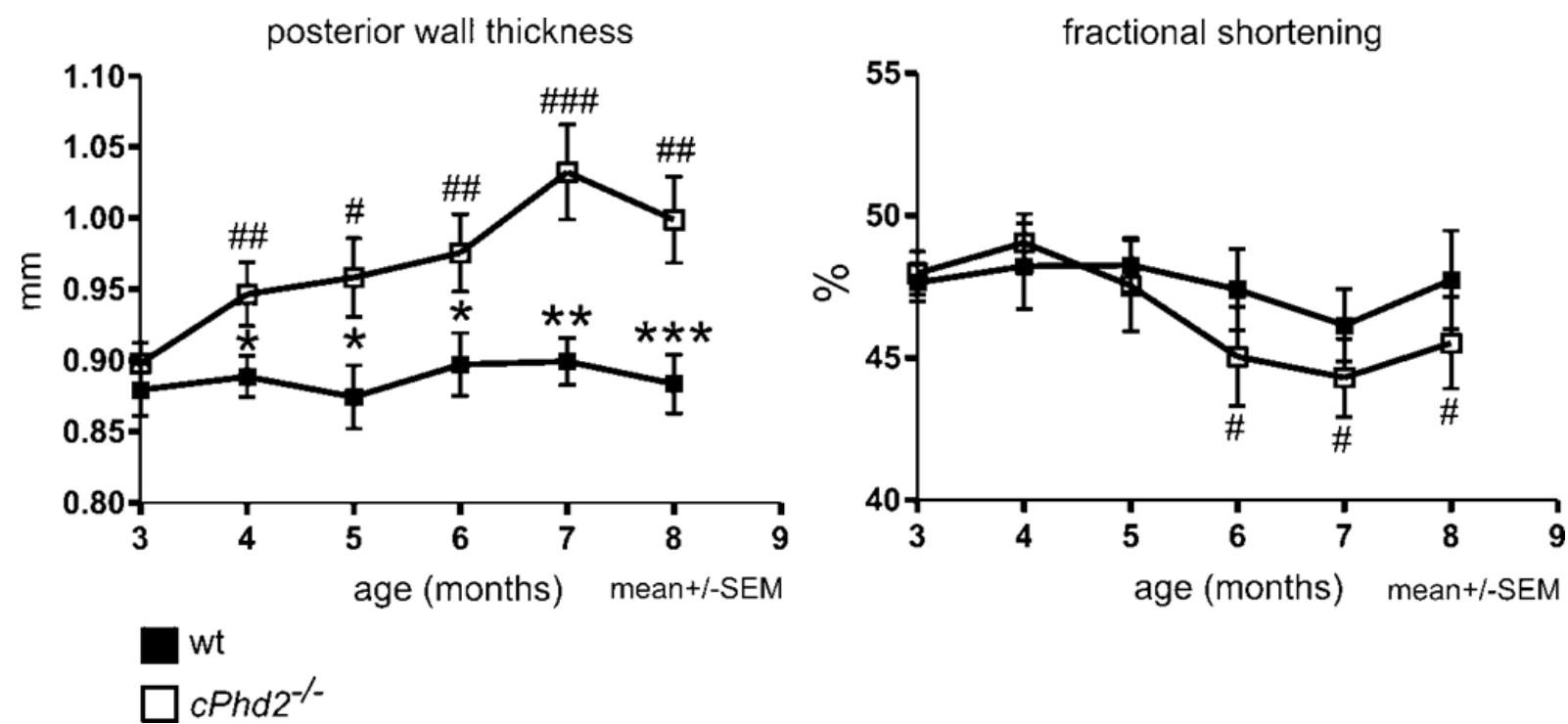

Fig.1 $\mathrm{CPhd2}^{-/-}$mice develop a hypertrophy with aging. A cohort of $w \mathrm{t}(\mathrm{n}=13)$ and $c P h d 2^{-/-}(\mathrm{n}=13)$ mice was followed over a time period of 8 months to determine long-term consequences of a PHD2 knock-out.

*, $\mathrm{p}<0.05 ;{ }^{* *}, \mathrm{p}<0.01 ;{ }^{* * *}, \mathrm{p}<0.001$ compared to wt; \#, $\mathrm{p}<0.05 ; \# \#, \mathrm{p}<0.01 ; \# \#, \mathrm{p}<0.001$ compared to 3 months

When analyzing the metabolism of 8 week and 8 month old $c P h d 2^{-/-}$and $w t$ littermates by PET analysis we could detect a shift towards glycolysis in 8 month old $c P h d 2^{-/-}$mice but not in 8 week old animals (fig. 2). 


\section{Unpublished Data}

\section{Fig.2}
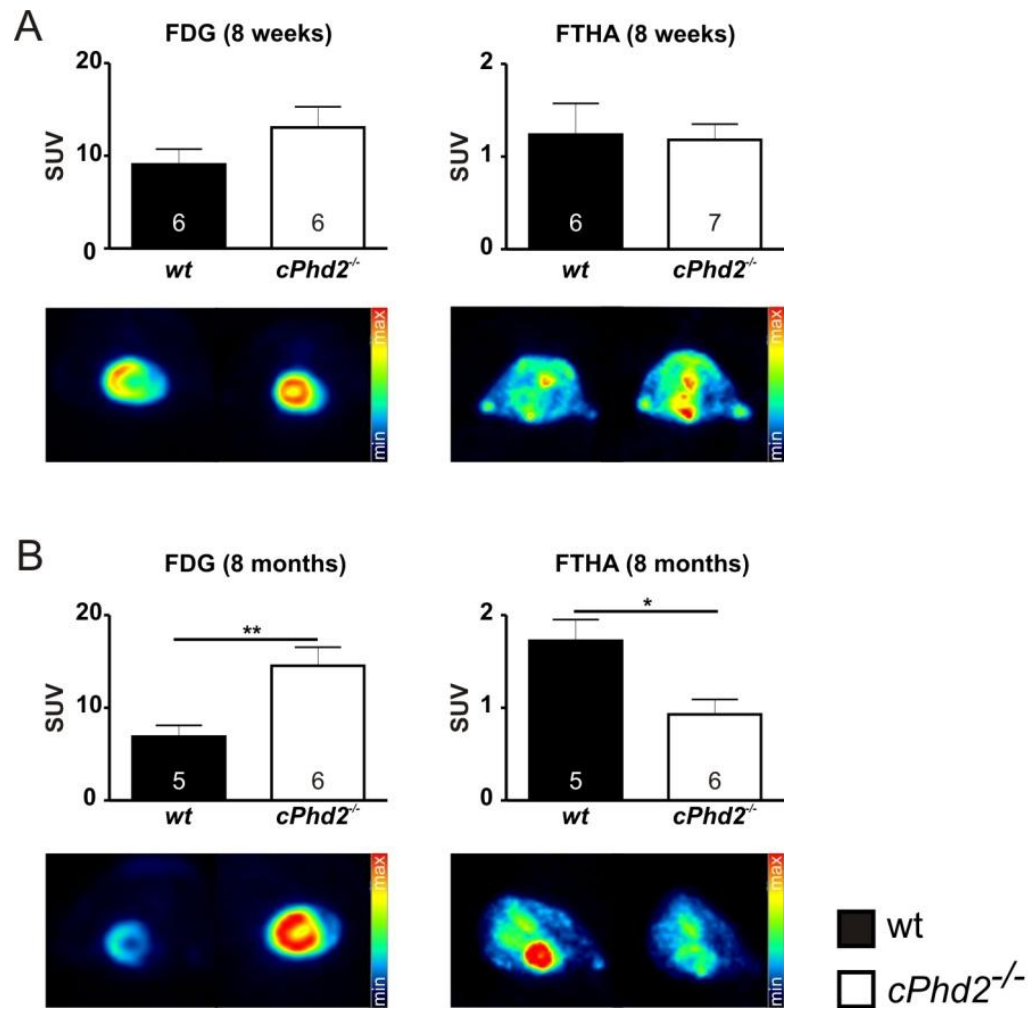

Fig. 2 The metabolism of 8 month old $c P h d 2^{-/-}$mice is shifted towards glycolysis. $\left[{ }^{18} \mathrm{~F}\right] \mathrm{FDG}$ uptake and $\left[{ }^{18} \mathrm{~F}\right] \mathrm{FTHA}$ uptake in (A) 8 week old and (B) 8 month old $c P h d 2^{-/}$and wt mice. Bar diagram shows cardiac SUV at 60 min. ${ }^{*}, \mathrm{p}<0.05 ;{ }^{*}, p<0.01$. Data represent mean \pm SEM. The number in the bars indicate the number of analyzed animals.

\section{Discussion}

Earlier on, we could show that $c P h d 2^{-/}$mice show a mild cardiac HIF-1 $\alpha$ stabilization with subsequent up-regulation of several HIF target genes. Amongst other genes, genes involved in metabolism were significantly up-regulated. Furthermore the capillary diameter was increased in hearts of $\mathrm{CPhd} 2^{\%}$ mice. Due to these changes $\mathrm{CPhd2} 2^{-/}$mice are primed to withstand acute ischemic insults. This could be shown by a smaller area at risk and a better preserved heart function three weeks after the ligation of the left anterior descending artery. Although these changes are beneficial on a short-term basis they have a detrimental outcome on a long-term basis. $c P h d 2^{-/}$mice develop a hypertrophy with aging (fig. 1) which can be partly explained by the observed in shift in metabolism towards glycolysis (fig. 2). As this same result (shift towards glycolysis and hypertrophy) can be also observed in HIF-1 $\alpha^{\text {tg }}$ mice (Hölscher et al., 2012) is can be assumed that the effects are HIF-mediated. 


\title{
4. Unpublished Data
}

\subsection{Cardiomyocyte-specific double knock-out of PHD2 and PHD3 leads to dilated cardiomyopathy and a premature death}

Marion Hölscher ${ }^{1}$, Ben Wielockx ${ }^{2}$, Georg Breier ${ }^{2}$, Dörthe M. Katschinski ${ }^{1 *}$, Anke Zieseniss ${ }^{1 *}$ (*both authors contributed equally)

(1) From the Department of Cardiovascular Physiology, Universitätsmedizin Göttingen, Georg-August University Göttingen and (2) from the Department of Pathology, TU Dresden

\begin{abstract}
Proly-4-hydroxylase domain enzymes (PHDs) are the most important enzymes that sense changes in oxygen concentration and directly control the cellular response by destabilizing the $\alpha$-subunit of the hypoxia-inducible factor (HIF), the master transcriptional regulator of the hypoxic response. Beforehand we have generated cardiomyocyte-specific PHD2 knockout mice $\left(c P h d 2^{-/}\right)$that showed a HIF-1 $\alpha$ stabilization with subsequent up-regulation of several HIF target genes. As PHD3 is a HIF target gene itself it was also up-regulated. To repress this regulatory feedback loop, we created cardiomyocyte-specific PHD2/PHD3 double knock-out mice $\left(c P H D 2 / 3^{--}\right)\left(P h d 2^{\text {flox/flox }} \times\right.$ Phd $\left.3^{\text {flox/flox }} \times M L C V C r e\right)$. These mice show signs of dilated cardiomyopathy that is already detectable at the age of eight weeks. This leads to premature death of the mice at the age of four to six months.
\end{abstract}

\section{Introduction}

The maintenance of oxygen homeostasis is critical to all aerobic organisms. In animals, complex systems have evolved to sense and to adapt to changes in cellular oxygen availability. The most important factor in response to low oxygen concentrations is the Hypoxia-Inducible Factor (HIF). HIF is a heterodimeric transcription factor consisting of one of three oxygen-sensitive $\alpha$-subunits (HIF- $1 \alpha$, HIF- $2 \alpha$ or HIF- $3 \alpha$ ) and the common oxygenindependently regulated $\beta$-subunit. Under normoxic conditions two proline residues (Pro 402 and $\mathrm{Pro}^{564}$ ) of the $\alpha$-subunit of HIF-1 are hydroxlated by prolyl-4-hydroxylase domain enzymes (PHDs). This hydroxylation marks HIF-1 $\alpha$ for ubiquitination by the VHL E3 Ligase which then leads to rapid degradation by the proteasome (Maxwell et al., 1999).

Up to now three PHD isoforms with known physiological functions have been identified, PHD1, PHD2 and PHD3. Based on its ubiquitous expression pattern and its dominant effect in normoxia, PHD2 seems to be the most important isoform. This assumption was further affirmed by the finding that PHD2 knock-out mice are not viable, as they die early between 


\section{Unpublished Data}

embryonic day 12.5 and 14.5, whereas PHD1 as well as PHD3 knock-out mice are viable (Takeda et al., 2006).

To gain further inside into the function of PHD2 in vivo two independent inducible PHD2 knock-out mice were generated (Minamishima et al., 2007; Takeda et al., 2007). These mice show, amongst other symptoms, signs of a dilated cardiomyopathy. However, these mice also have an increased hematocrit, thus it is not clear if the observed cardiac phenotype is a primary effect or if it is due to the hyperviscosity.

Therefore we have previously generated cardiomyocyte-specific PHD2 knock-out mice $\left(c P h d 2^{-/}\right.$) (Hölscher et al., 2011). These mice show slightly stabilized HIF-1 $\alpha$ protein levels with subsequent up-regulation of HIF target genes. These mice are protected from acute myocardial infarction which can be displayed by a smaller area of necrosis (AON) and a smaller area at risk (AAR) six hours after ligation of the left anterior descending artery. Furthermore the heart function of these mice is better preserved three weeks after the infarction. The response to an elevated afterload, as induced by transverse aortic constriction, however did not differ in $\mathrm{CPhd}^{-/}$mice compared to wt mice. As PHD3 is a HIF target gene itself, it is also significantly up-regulated (about 8-fold on mRNA level compared to $w t$ littermates).

Minamishima et al. (2009) could show that PHD2 and PHD3 are partially redundant in vivo with PHD3 to some extent compensating when PHD2 is inactive. Thus the effects caused by the PHD2 knock-out in the $\mathrm{CPhd2} 2^{-/}$mice are attenuated by the compensatory up-regulation of PHD3. To analyze the cardiac function of a double knock-out of PHD2 and PHD3 we generated cardiomyocyte-specific knock-out mice by crossing Phd $2^{\text {flox/flox }} \times \mathrm{Phd}^{\text {flox/flox }}$ mice with MLCVvCre/+ mice. These mice show a significant down-regulation of PHD2 and PHD3 and a subsequent up-regulation of several HIF target genes. Furthermore, the PHD2/PHD3 double knock-out mice develop a dilated cardiomyopathy that leads to premature death.

\section{Material and Methods}

Animal Experimentation and Echocardiography-Animal experimentation was performed with $c P h d 2 / 3^{--}$mice and littermate $P h d 2 / 3 w t$ control mice. All protocols regarding animal experimentation were conducted according to the German animal protection laws and approved by the responsible governmental authority (Niedersächsisches Landesamt für Verbraucherschutz und Lebensmittelsicherheit in Oldenburg; animal experimentation 


\section{Unpublished Data}

numbers 33.942502-04-10/0024 and 33.9-42502-04-10/0069). Echocardiography and measurement of left ventricular end systolic diameter (LVESD), left ventricular end diastolic diameter (LVEDD) and fractional shortening (FS) were performed as described by Silter et al. (2009).

Mice- All animals in this study were backcrossed to C57BL/6 mice at least five times. $P h d 2^{\text {flox/flox }} \times M L C v C r e /+$ mice were crossed with $P h d 3^{\text {flox/flox }} \times M L C v+/+$ (or vice versa) to

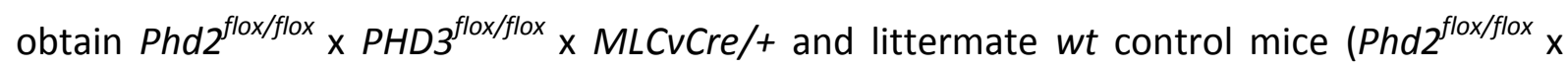
$P h d 3^{\text {flox } / f l o x} \times \mathrm{MLCV}+/+$ ) within two generations.

Mice were genotyped by PCR using the following primers: Cre forward, 5'CGTACTGACGGTGGGAGAAT-3' and Cre reverse, 5'-CGGCAAAACAGGTAGTTA-3'; PHD2 forward, 5'-CTCACTGACCTACGCCGTGT-3' and PHD2 reverse, 5'-CGCATCTTCCATCTCCATTT-3'; PHD3 forward, 5'-ATGGCCGCTGTATCACCTGTAT-3' and PHD3 reverse, 5' - CCACGTTAACTCTAGAGCCACTGA-3'.

The successful deletion of PHD2 and PHD3 in the floxed mice was verified by qRT-PCR analysis.

Protein Extraction and Immunoblot Analyses- Protein extraction and immunoblot analyses were performed as previously described by Hölscher et al. (2011).

qRT-PCR Analyses-After RNA extraction reverse transcription (RT) was performed with $2 \mu \mathrm{g}$ (left ventricle) or $1 \mu \mathrm{g}$ (atrium) of RNA and a first strand cDNA synthesis kit (Fermentas, St. Leon-Rot). mRNA levels were quantified by using $0.5 \mu$ of the CDNA reaction and a SYBR Green qPCR reaction kit (Clontech) in combination with a MX3000P light cycler (Stratagene). The initial template concentration of each sample was calculated by comparison with serial dilutions of a calibrated standard in reference to $\mathrm{mS} 12$. Primers were as follows: $\mathrm{mS} 12$ forward, 5'-GAAGCTGCCAAGGCCTTAGA-3' and mS12 reverse, 5'-AACTGCAACCAACCACCTTC3'; PHD1 forward, 5'-GCTAGGCTGAGGGAGGAAGT-3' and PHD1 reverse, 5'- TCTACCCAGGCAATCTGGTC-3'; PHD2 forward, 5'-TCTATTTTGCCAGACCTGTCACC-3' and PHD2 reverse, 5'-GAAGCAACACGTCGTCACTCACT-3'; PHD3 forward, 5'-GGCCGCTGTATCACCTGTAT-3' and PHD3 reverse, 5'-TTCTGCCCTTTCTTCAGCAT-3'; HIF-1 $\alpha$ forward, 5'-TCAAGTCAGCAACGTGGAAG-3' and HIF-1 $\alpha$ reverse, 5'-TATCGAGGCTGTGTCGACTG-3'; phosphofruktokinase 1 (Pfkl) forward, 5'-ACGAGGCCATCCAGCTCCGT-3' and Pfkl reverse, 5'-TGGGGCTTGGGCAGTGTCCT-3'; glucose transporter-1 (Glut1) forward, 5'-TGGCCTTGCTGGAACGGCTG-3' and Glut1 reverse, 


\section{Unpublished Data}

5'-TCCTTGGgCtGCAGgGAGCA-3'; CA IX (Carbonic anhydrase 9) forward, 5'-GGGTGTCATCTGGACTGTGTT-3' and CA IX reverse, 5'-CTTCTGTGCTGCCTTCTCATC-3'; $\alpha$-skeletal actin forward, 5'-GGCACCCAGGGCCAGAGTCA-3' and $\alpha$-skeletal actin reverse, 5'-CACGATGCCGGTGGTACGGC-3'.

Statistical analysis- Data were analysed by 2-tailed Student's unpaired $t$ test or in case of repeated echocardiography analysis of aging mice as paired $t$ test and presented as mean \pm SEM. A $P$ value less than 0.05 was considered statistically significant.

Kaplan-Meier survival curves have been made using GraphPad Prism 4 (GraphPad Software, San Diego, CA) and analyzed using log-rank test: **, p<0.01

\section{Results}

Generation of cardiomyocyte-specific PHD2/PHD3 knock-out mice- For generating cardiomyocyte-specific PHD2/PHD3 knock-out mice (cPhd2/3/-) Phd2 flox/flox $\times$ MLCvCre/+ mice were crossed with $P h d 3^{\text {flox/flox }} \times \mathrm{MLCV}+/+$ to obtain $c P h d 2 / 3^{-/-}$mice within two generations. The expression of the MLCVCre starts at embryonic day E7.5 which coincidences with the earliest stages of ventricular specification (Chen et al., 1998). Exons 2 and 3 of the Phd 2 gene and Exon 2 of the Phd3 gene are flanked by loxP-sites. The MLCV-driven Cre recombinase allows the recombination of the floxed exons only in ventricular cardiomyocytes (fig. 1A). Analyzing the mRNA levels of PHD1, PHD2 and PHD3 it could be shown that the levels of PHD2 and PHD3 are significantly reduced in the ventricle but not in the atrium (fig. 1B). As the mRNA is isolated from whole tissues, i.e. ventricles and atria, and not from isolated cardiomyocytes the knock-out is not $100 \%$ in the sample but within an expected dimension. The mRNA-levels of the non-hypoxia inducible PHD1 remain unchanged. Intriguingly, HIF-1 $\alpha$ is significantly down-regulated on mRNA level (fig. 1C). Despite this down-regulation, HIF-1 $\alpha$ protein levels show a stabilization of HIF-1 $\alpha$ in the left ventricle. This stabilization is in a similar range as in $\mathrm{CPhd}^{-\%}$ mice but seemingly less than in Hif- $1 \alpha^{\text {tg }}$ mice (fig. 1D). Representative HIF target genes were analyzed on mRNA level. Genes involved in glucose metabolism (Glut1, Pfkl), pH control (CA IX) and a constituent of the contractile apparatus ( $\alpha$-skeletal actin) are significantly up-regulated (fig. 1E). This demonstrates that the HIF pathway is activated in the cardiomyocytes of $c P h d 2 / 3^{--}$mice in spite of the reduction of HIF-1 $\alpha$ on mRNA level. 


\section{Unpublished Data}

Fig.1

A

PHD2

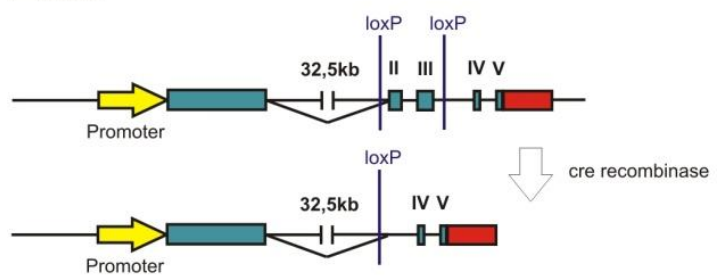

PHD3

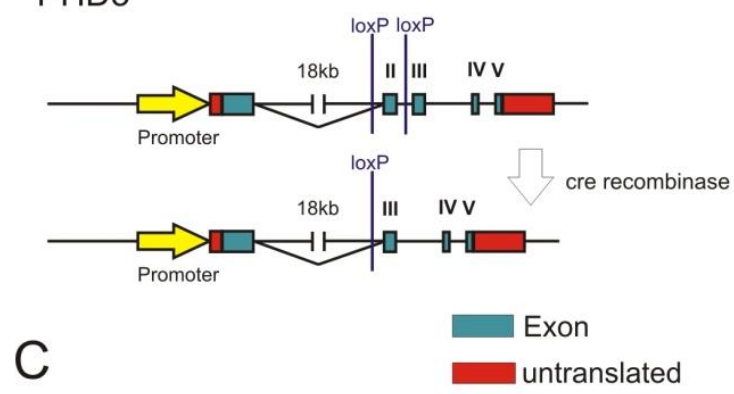

B

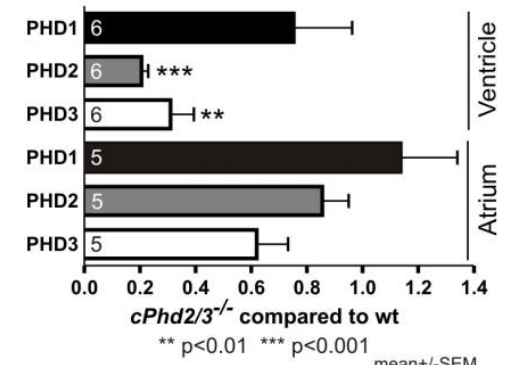

$\mathrm{D}$
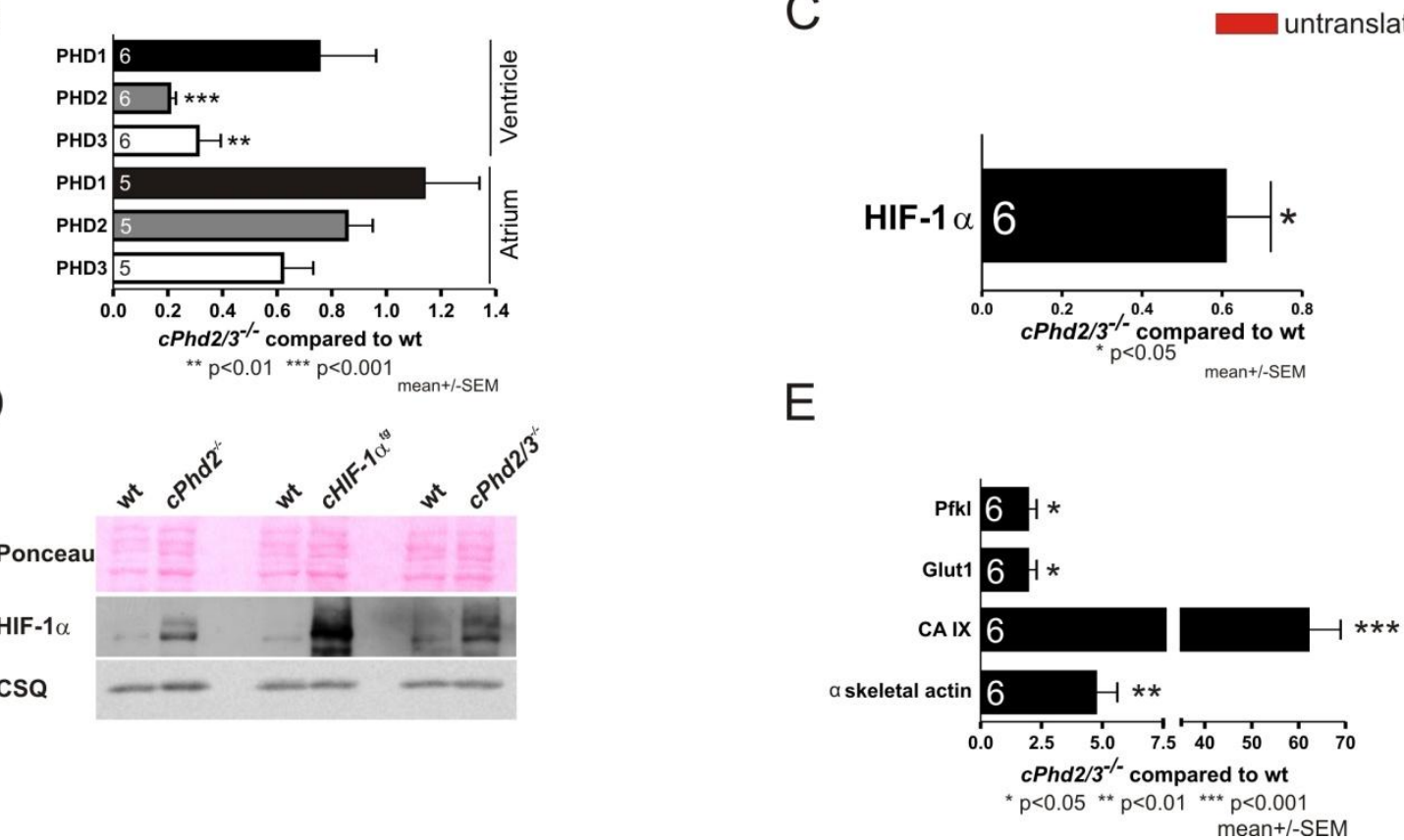

Fig.1 Generation and analyses of $\boldsymbol{c P h d 2} / 3^{-/}$mice. (A) Schematic description of the gene targeting strategy is shown. In the targeted Phd 2 locus exons 2 and 3 and in the targeted Phd 3 locus exon 2 are flanked by two loxP sites. These flanked exons of Phd 2 and $P h d 3$ are deleted by Cre-mediated recombination by crossing Phd $2^{\text {flox/flox }} \times$ Phd $3^{\text {flox/flox }}$ mice with MLCvCre mice. (B) qRT-PCR analysis confirmed the significant reduction of PHD2 and PHD3 mRNA transcripts in the left ventricles but not in the atria of 8 week old $c P h d 2 / 3^{--}$mice compared with wt littermates. PHD1 mRNA levels were unchanged in the ventricle and in the atrium. In total, 6 (atrium: 5) wt and 6 (atrium: 5) cPhd $2 / 3^{-1}$ mice were analyzed. ${ }^{* *}, p<0.01 ;{ }^{* * *}, p<0.001$. Data represent mean $\pm \mathrm{SEM}$. (C) HIF-1 $\alpha$ mRNA levels in left ventricle were determined by qRT-PCR analysis and showed a significant downregulation. ${ }^{*}, p<0.05$ Data represent mean \pm SEM. (D) HIF-1 $\alpha$ protein was detected by Western blot analysis. Protein extracts prepared from left ventricles of 8 week old Phd $2 \mathrm{wt}$ and $c P h d 2^{-/}$, Hif- $1 \alpha^{w t}$ and Hif- $1 \alpha^{t g}$ and $c P h d 2 / 3$ wt and $c P h d 2 / 3^{-\%}$ mice were analyzed with anti-HIF- $1 \alpha$ and anticalsequestrin (CSQ) antibodies, confirming that HIF-1 $\alpha$ is stabilized in the left ventricles. Ponceau staining and CSQ show equal loading. (E) Representative HIF target genes were analyzed by qRT-PCR. Pfkl, Glut1, $\alpha$-skeletal actin and CA IX showed a significant up-regulation on mRNA level in left ventricles of 8 week old $c P h d 2 / 3^{-\%}$ mice in comparison to wt littermates. ${ }^{*}, p<0.05 ;{ }^{* *}, p<0.01 ;{ }^{* * *}$, $p<0.001$. Data represent mean \pm SEM. The number in the bars indicate the number of analyzed animals. 


\section{Unpublished Data}

$\mathrm{cPhd} 2 / 3^{--}$mice develop dilated cardiomyopathy with aging which leads to a premature death. I was able to show earlier (Hölscher et al., 2012) that cardiac-specific HIF-1 $\alpha$ transgenic mice develop heart failure with aging or when challenged by increased mechanical load. $c$ Phd $2^{-/}$mice, which show only mild levels of HIF-1 $\alpha$ stabilization did not respond differently to elevated mechanical load than their wt littermates (Hölscher et al., 2011). However these mice also develop cardiac hypertrophy with aging, though, without a loss in heart function (see chapter 4.1, fig. 1). To determine the consequences of a double knock-out of PHD2 and PHD3 in cardiomyocytes on the cardiac function $c P h d 2 / 3^{-}$mice underwent echocardiography every two weeks between the age of eight and eighteen weeks. Representative echocardiographic recordings are shown in figure 2B (B-mode; brightness mode) and in figure $2 \mathrm{C}$ (M-mode; motion mode). $\mathrm{CPhd} 2 / 3^{-/}$mice already show an inferior heart function at the age of eight weeks that worsens over time (fig. 2A). At the age of 4.5 months, $c P h d 2 / 3^{-/}$mice show an enlargement of the heart (fig. 2D), which is also shown by an increased end-diastolic diameter (fig. 2A). Wt littermates on the contrary, did not show any signs of a weakened heart function, an increase in heart size or premature death. $c P h d 2 / 3^{-}$mice die significantly earlier than wt mice with a median survival of 21 weeks (fig. 2E). 


\section{Unpublished Data}

Fig.2

A

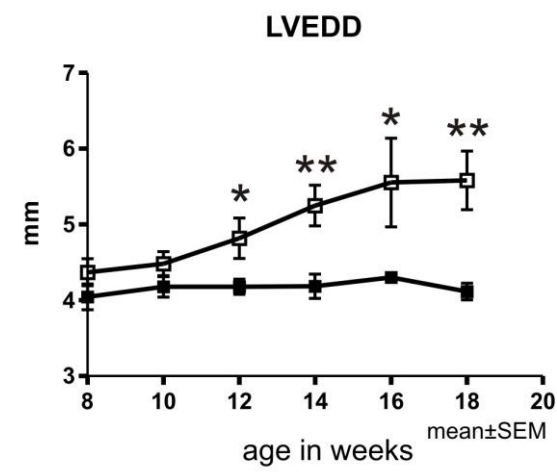

LVESD

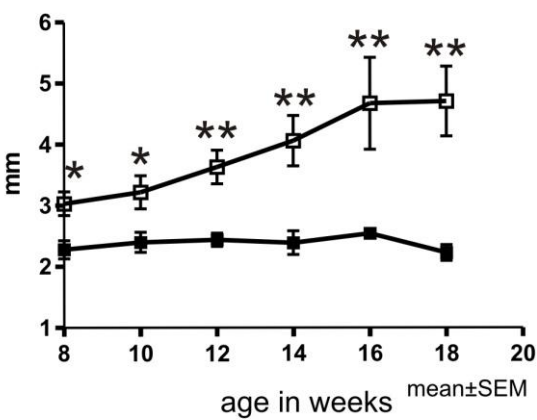

FS

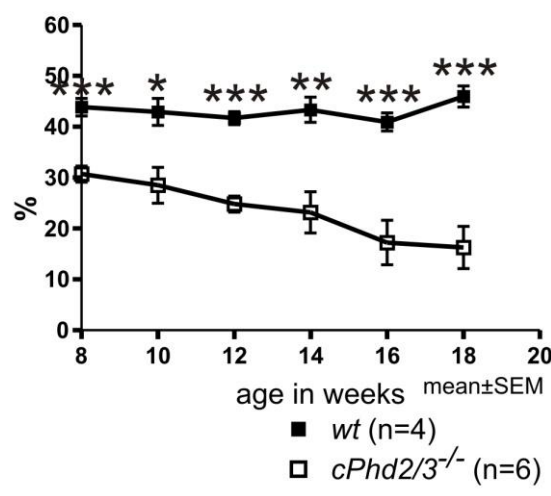

B

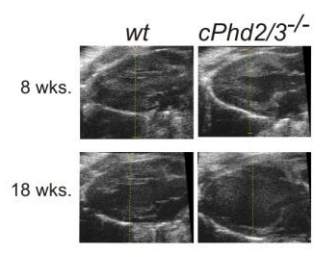

C wt cPhd $2 / 3^{-/-}$

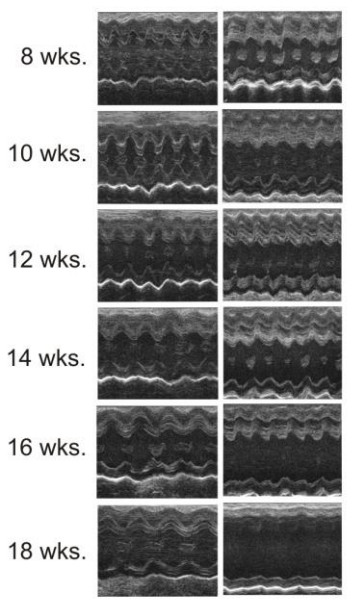

D wt $\quad c P h d 2 / 3^{-/-}$

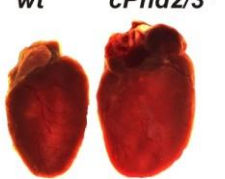

E

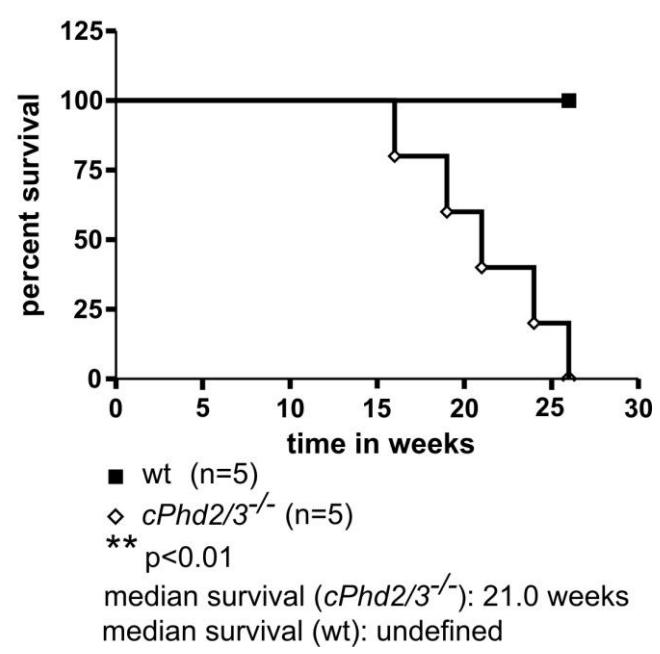

Fig. 2 Cardiomyocyte-specific deficiency of PHD2 and PHD3 leads to dilated cardiomyopathy. cPhd $2 / 3^{-1}(n=6)$ and wt littermates $(n=4)$ were analyzed by echocardiography every two weeks between the age of eight and eighteen weeks. (A) Left ventricular end-diastolic diameter (LVEDD) and left ventricular end-systolic diameter (LVESD) were determined. Fractional shortening (FS) of the left ventricle as a marker for heart function was defined as (FS= LVEDD - LVESD/ LVEDD $\times 100$ ). Graphs represent mean \pm SEM. ${ }^{*}, p<0.05 ; * *, p<0.01 ; * * *, p<0.001$. Echocardiographic images recorded from parasternal long axis view of a $c P h d 2 / 3^{--}$and a wt mice; (B) B-Mode, (C) M-Mode. (D) Explanted hearts of a $\mathrm{CPhd} 2 / 3^{-/}$and a wt mouse at the age of eighteen weeks; (E) Kaplan-Meier survival curve for $c P h d 2 / 3^{--}$and wt littermates. Wt mice live significantly longer than $c P h d 2 / 3^{--}$mice which die around the age of 21 weeks. 


\section{Unpublished Data}

\section{Discussion}

HIF-1 $\alpha$ was shown to be critically involved in cardioprotection (Cai et al., 2003; Date et al. 2005). Previously it has been shown by our group (Hölscher et al., 2011) and others (e.g. Hyvärinen et al., 2010) that stabilization of HIF-1 $\alpha$ mediated by a knock-out of PHD2 is beneficial in the setting of acute myocardial infarction. Currently small molecule inhibitors are being developed to stabilize HIF-1 $\alpha$ in order to treat diseases related to hypoxia like anemia, stroke, or myocardial infarction (Katschinski, 2009; Myllyharju, 2009; Yan et al., 2010).

To gain insight into the outcome of a double knock-out of PHD2 and PHD3 in the heart, $c P h d 2 / 3^{-/}$mice were created. These mice show a significant down-regulation of PHD2 and PHD3 in the ventricular cardiomyoctes compared to $w t$ animals but unchanged levels of PHD1. Interestingly, $c P h d 2 / 3^{-/}$show a significant down-regulation of HIF-1 $\alpha$ mRNA levels. In a recent report it was shown that in HMEC-1 cells sustained hypoxia progressively decreases HIF-1 $\alpha$ mRNA while HIF-1 $\alpha$ protein levels rapidly peak after $3 \mathrm{~h}$ and then slowly decay (Chamboredon et al., 2011). Therefore it can be speculated that in $c P h d 2 / 3^{-/-}$hearts an extreme hypoxic environment is mimicked so that the HIF-1 $\alpha$ mRNA levels are decreased. Representatively analyzed HIF target genes show a significant up-regulation to a similar extent as in $\mathrm{CPhd}^{-/}$mice (e.g. Glut1 2.5-fold up-regulated in $\mathrm{CPhd} 2^{-/}$and $c P h d 2 / 3^{-/}$mice) but less than in Hif-1 $\alpha^{\text {tg }}$ mice (e.g. Glut1 5-fold up-regulated).

$c P h d 2 / 3^{-/}$mice show already at a very young age (eight weeks) signs of cardiomyopathy and a constriction of their heart function. The impairment of the heart function of $c P h d 2 / 3^{-/}$ mice finally leads to a premature death at the age of maximal six months.

HIF-1 $\alpha$ transgenic mice (either over-expressing the wild-type HIF-1 $\alpha$ isoform or a modified form which cannot be hydroxylated as amino acids critical to HIF-1 $\alpha$ degradation (i.e. Pro ${ }^{402} \mathrm{Ala}$, Pro ${ }^{564} \mathrm{Ala}$ and $\mathrm{Asn}{ }^{803} \mathrm{Ala}$ ) were substituted) (Hölscher et al., 2012; Bekeredjian et al., 2010) develop a hypertrophy with aging or when challenged by elevated mechanical load. As $c P h d 2 / 3^{-/}$mice unexpectedly do not show a more profound HIF-1 $\alpha$ stabilization than Hif- $1 \alpha^{\text {tg }}$ mice the HIF-1 stabilization could still be a contributing factor for the premature death but might not be the main reason. This will be analyzed in follow-up studies by creating cardiomyocyte-specific PHD2/PHD3/HIF-1 $\alpha$ triple knock-out mice. If the HIF-1 $\alpha$ stabilization is causative for the premature death of the $c P h d 2 / 3^{-/}$mice the cPhd2/3/HIF-1 $\alpha^{-/-}$triple knock-out mice should show a normal life span. 


\section{Unpublished Data}

Despite the promising perspective in developing drugs to mediate the cardioprotective effect of HIF-1 $\alpha$, it is indispensable to gain deeper insight into the specific functions of the PHD isoforms to create isoform specific PHD inhibitors as at least a double knock-out of PHD2 and PHD3 in cardiomyocytes has a detrimental outcome. Furthermore other pathways than the HIF pathway that are regulated by the PHDs need to be studied to further elucidate the consequences of this inhibition.

Acknowledgments-We thank Sabine Krull and Annette Hillemann for expert technical assistance.

\section{References}

Chamboredon S, Ciais D, Desroches-Castan A, Savi P, Bono F, Feige JJ, Cherradi N. Hypoxiainducible factor-1 $\alpha$ mRNA: a new target for destabilization by tristetraprolin in endothelial cells.Mol Biol Cell 18:3366-78.

Chen J, Kubalak SW and ChienKR (1998).Ventricular muscle-restricted targeting of the RXRa gene reveals a non-cellautonomous requirement in cardiac chamber morphogenesis. Development 125:1943-1949

Bekeredjian R, Walton CB, MacCannell KA, Ecker J, Kruse F, et al. 2010 Conditional HIF-1 $\alpha$ Expression Produces a Reversible Cardiomyopathy. PLoS ONE 5(7): e11693

Hölscher M, Silter M, Krull S, von Ahlen M, Hesse A, Schwartz P, Wielockx B, Breier G, Katschinski DM, Zieseniss A (2011). Cardiomyocyte-specific prolyl-4-hydroxylase domain 2 knock out protects from acute myocardial ischemic injury. J. Biol. Chem. 286:11185-11194

Hölscher $M$, Schäfer K, Krull S, Farhat K, Hesse A, Silter M, Lin Y, Pichler BJ, Patricia Thistlethwaite P, El Armouche A, Maier LS, Katschinski DM, Zieseniss A (2012) Unfavourable consequences of chronic cardiac HIF-1 $\alpha$ stabilization. Cardiovasc Res 94:77-86

Hyvärinen J, Hassinen IE, Sormunen R, Mäki JM, Kivirikko KI, Koivunen P, and Myllyharju, J (2010). Hearts of hypoxia-inducible factor prolyl 4-hydroxylase-2 hypomorphic mice show protection against acute ischemia-reperfusion injury. J Biol Chem 285:13646-13657

Katschinski DM (2009). In vivo functions of the prolyl-4-hydroxylase domain oxygen sensors: direct route to the treatment of anaemia and the protection of ischaemic tissues. Acta Physiol 195:407-414

Maxwell PH, Wiesener MS, Chang GW, Clifford SC, Vaux EC, Cockman ME, Wykoff CC, Pugh CW, MaherER, and Ratcliffe PJ (1999). The tumour suppressor protein VHL targets hypoxiainducible factors for oxygen-dependent proteolysis. Nature 399:271-275. 


\section{Unpublished Data}

Minamishima YA, Moslehi J, Bardeesy N, Cullen D, Bronson RT, Kaelin WG Jr (2007). Somatic inactivation of the PHD2 prolyl hydroxylase causes polycythemia and congestive heart failure; Blood 111:3236-44.

Minamishima YA, Moslehi J, Padera RF, Bronson RT, Liao R and Kaelin Jr WG (2009). A Feedback Loop Involving the Phd3 Prolyl Hydroxylase Tunes the Mammalian Hypoxic Response In Vivo. Mol Cell Biol 29:5729-5741

Myllyharju J. (2009). HIF Prolyl 4-Hydroxylases and their Potential as Drug Targets. Curr Pharm Des 15:3878-3885

Takeda K, Cowan A, Fong GH (2007). Essential role for prolyl hydroxylase domain protein 2 in oxygen homeostasis of the adult vascular system. Circulation 116:774-81

Takeda K, Ho V, Takeda H, Duan L, Nagy A, and Fong G (2006). Placental but Not Heart Defects Are Associated with Elevated Hypoxia-Inducible Factor $\alpha$ Levels in Mice Lacking Prolyl Hydroxylase Domain Protein 2. MCB 22:8336-8346

Silter M, Kögler H, Zieseniss A, Wilting J., Schäfer K, Toischer K, Rokita AG, Breves G, Maier LS, Katschinski DM (2010). Impaired Ca2+-handling in HIF-1 $\alpha+/-$ mice as a consequence of pressure overload. Pflugers Arch - Eur J Physiol 459:569-577

Yan L, Colandrea VJ, and Hale JJ (2010). Prolyl hydroxylase domain-containing protein inhibitors as stabilizers of hypoxia-inducible factor: small molecule-based therapeutics for anemia. Expert Opin Ther Pat 20:1219-1245 


\section{Discussion}

\section{Discussion}

The maintenance of cellular oxygen homeostasis is critical to all aerobic organisms in order to produce energy for cell survival and reproduction. The master regulator in response to decreased oxygen availability is HIF. HIF is a heterodimeric transcription factor consisting of one of three oxygen-sensitive $\alpha$-subunits and an oxygen-stable $\beta$-subunit. The molecular basis for the oxygen-dependent regulation of the $\alpha$-subunit is the hydroxylation of two distinct proline residues. This hydroxylation is committed by a family of oxygen-, iron- and 2oxoglutarate dependent PHD enzymes that have been first described in 2001 (Bruick and McKnight, 2001; Epstein et al., 2001; Ivan et al., 2002). So far three members of this family with known physiological functions have been identified- PHD1, PHD2 and PHD3. The PHDs have many features in common but they show some differences in expression pattern, catalytic properties and physiological role (see table 1). PHD inhibition has been proven to activate the HIF pathway in vitro and in vivo (Warnecke et al., 2003). The knowledge about PHD isoform specific functions is important for the ongoing development of small molecule PHD inhibitors. The first PHD inhibitors are currently tested in preclinical and clinical trials for the treatment of anemia and for cytoprotection (Yan et al., 2010).

HIF-1 has been attributed a central role in a variety of cell functions by transcriptional regulation of a multitude of genes involved in e.g. angiogenesis, vasotonus regulation, glucose metabolism, viability and $\mathrm{pH}$ control.

In the course of time HIF-1 became a promising candidate in mediating cardioprotection. Cai et al. demonstrated a positive effect of hypoxia in the setting of acute myocardial infarction for the first time in 2003. Rats were exposed to intermittent hypoxia (5 cycles: 6 minutes of $6 \% \mathrm{O}_{2}$ followed by room air for 6 minutes); control rats did not undergo this procedure. Afterwards explanted hearts perfused in Langendorff mode showed a smaller infarction area compared to control hearts. Since then several studies followed that could show that HIF-1 is critical involved in regulation of cellular heart functions (for review see: Tekin et al., 2010).

Despite the fact that PHD inhibitors are already currently being designed in order to treat or attenuate the outcome of acute myocardial infarction (and other diseases like stroke, anemia, cancer, etc.) in the nearby future a lot of open questions regarding HIF-1 $\alpha$ stabilization still remain.

The aims of this thesis were to analyze the cardiomyocyte-specific functions of PHD2 and PHD3 and to address the effects of cardiac HIF-1 $\alpha$ stabilization. 


\section{Discussion}

\subsection{Beneficial effects of PHD2 knock-out, respectively HIF-1 $\alpha$ stabilization}

Myocardial infarction is the death of heart muscle caused by sudden blockage of a coronary artery perfusion. If blood flow is not restored to the heart muscle within 20 to 40 minutes, irreversible death of the heart muscle will begin to occur. In the last couple of years evidence thickened that HIF-1 is an important mediator of cardioprotection. This was beforehand also shown by Kido et al. (2005) for the HIF-1 $\alpha$ transgenic mice $\left(H i f-1 \alpha^{\mathrm{tg}}\right.$ ) that were further analyzed in this thesis.

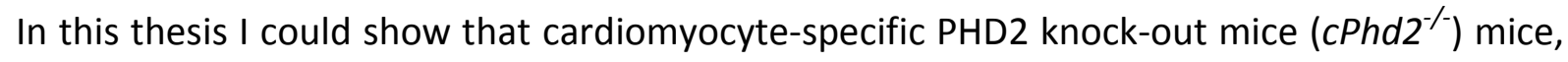
which show elevated cardiac HIF-1 $\alpha$ protein levels, had significantly smaller areas at risk and areas of necrosis as well as a better preserved heart function after a permanent ligation of the left anterior descending artery (LAD) (Hölscher et al., 2011, fig. 8). Furthermore I could demonstrate a better cell survival in the infarcted myocardium of $\mathrm{CPhd} 2^{-/}$mice compared to wt mice with significant less apoptotic cells in $c P h d 2^{-/-}$mice (Hölscher et al., 2011, fig. 8).

In Hif- $1 \alpha^{\text {tg }}$ mice I could show a significant up-regulation of several vasotonus regulating genes. When determining the area and the number of capillaries via CD31 staining I could show that the number of capillaries is the same in Hif- $1 \alpha^{\text {tg }}$ and wt littermates, the area of the capillaries in Hif- $1 \alpha^{\text {tg }}$ mice, however, is significantly increased which allows a better blood flow into the heart (Hölscher et al., 2012, fig. 2). The same effect could be shown for cPhd $2^{-/}$mice despite the non-changed expression of vasotonus regulating genes (Hölscher et al., 2011, fig. 6). The increase of capillary diameter and therefore an expected improvement in oxygen supply might be at least one reason for the cardioprotective effect following a myocardial infarction.

The heart can use different metabolic fuels, including fatty acids, glucose, lactate, ketones, and amino acids. In healthy condition fatty acids are the preferred fuel, accounting for up to $90 \%$ of the total acetyl-CoA provided to cardiac mitochondria. Comparing fatty acids (e.g. palmitate) and glucose the complete oxidation of one mol palmitate generates 105 molecules of ATP and consumes 46 atoms of oxygen. Oxidation of one mol glucose in contrast generates 31 molecules of ATP and consumes twelve atoms of oxygen. Thus the oxidation of fatty acids generates more ATP but it comes at the cost of higher $\mathrm{O}_{2}$ consumption (Lopaschuk et al., 2010).

During fetal stages glucose is used as the primary energy substrate whereas rates of fatty acid oxidation are low. After birth a switch towards fatty acid oxidation occurs. If the 


\section{Discussion}

newborn heart is exposed to volume overload hypertrophy this switch fails to appear (Stanley et al., 2005).

Glucose is central for energy production in the ischemic heart when the lack of oxygen induces a shift to anaerobic metabolism with subsequent stimulation of glucose uptake. There is evidence from animal studies that stimulation of carbohydrate oxidation with simultaneous inhibition of fatty acid oxidation can improve the heart function (Stanley et al., 2004). In hearts of $c P h d 2^{--}$and Hif- $1 \alpha^{t g}$ mice genes regulating glycolysis are significantly upregulated (e.g. Glut1, Pfkl) which goes along with higher rates of glucose uptake and lower fatty acid uptake as determined by PET analysis (Hölscher et al., 2012, fig. 3; chapter 4.1, fig. 2). In addition to the changes in capillary structure, this change in metabolism most likely also contributes to the cardioprotective effect after myocardial infarction. The hearts of these mice are likely pre-conditioned to resist ischemic conditions.

Furthermore, the calcium handling of cells is a critical component for proper heart function. Despite the decrease of SERCA2a in hearts of Hif- $1 \alpha^{\text {tg }}$ mice we observed changes in calcium handling with increased intracellular $\mathrm{Ca}^{2+}$ - transients and a faster $\mathrm{Ca}^{2+}$-decline. This leads to the assumption that the down-regulation of SERCA2a might be counter-regulated by the observed increased PLB-phosphorylation (Hölscher et al., 2012, fig. 4).

Taken together the changes in metabolism, apoptosis, calcium handling and capillary area in cPhd $2^{-/}$and Hif- $1 \alpha^{\text {tg }}$ mice can be interpreted as improved oxygen supply and energy saving mechanisms which protects the heart in case of an acute ischemic insult.

\subsection{Detrimental effects of PHD2 knock-out, respectively HIF stabilization}

An important parameter for the application of potential PHD inhibitory drugs in order to treat ischemic diseases will be the duration of the treatment. As shown in this thesis develop Hif- $1 \alpha^{t g}$ (Hölscher et al., 2012, fig. 5) as well as $c P h d 2^{-/-}$mice (Chapter 4.1, fig. 1) a cardiac hypertrophy with aging which goes along with non-lethal heart failure up to the age of eight months.

Cardiac hypertrophy is a thickening of the heart muscle which results in decreased size of the chamber of the heart, including the left and right ventricle. Hypertrophy can occur pathologically in response to diverse stimuli including hypertension, valve disease, myocardial infarction and genetic mutations, or it can occur physiological in response to regular physical activity or chronic exercise training (McMullen and Jennings, 2007). While 


\section{Discussion}

the remodeling of the heart during developing pathological hypertrophy is irreversible it is fully reversible in the setting of physiological hypertrophy (Mihl et al., 2008).

Pathologic cardiac hypertrophy is associated with an increased risk of angina, heart attack or even heart failure. In a mouse model, pathological cardiac hypertrophy can be triggered by transverse aortic constriction. Consequences of this hypertrophy can be a dilatation of the ventricle which finally leads to heart failure. A developing mismatch between the number of capillaries and the size of cardiomyocytes which leads to myocardial hypoxia goes along with cardiac hypertrophy. The impact of HIF-1 on cardiac hypertrophy is discussed controversially in the literature. Several studies revealed a critical involvement of HIF-1 in the regulation of cardiac hypertrophy and cardiac function occurring in response to an elevated workload. Silter et al. (2009) could show that HIF-1 $\alpha^{+/-}$mice developed severe heart failure after TAC as revealed by a significantly reduced fractional shortening whereas HIF- $1 \alpha^{+/+}$mice did not. The extent of cardiac hypertrophy on the contrary was similar in both genotypes. As the calcium handling of cardiomyocytes is critically important for heart function it was analyzed in the mice. On the single cell level, cells from $\mathrm{HIF}^{+/-}$mice showed reduced myocyte shortening, decreased intracellular $\mathrm{Ca}^{2+}$-transients and $\mathrm{SR}-\mathrm{Ca}^{2+}$ content in myocytes in comparison to $\mathrm{HIF}^{+/+}$mice.

Huang et al. (2004) demonstrated a mild hypertrophy with increased wall thickness and heart to body weight ratio in resting constitutive cardiac-specific Hif-1 $\alpha$ knock-out mice despite of cardiac hypovascularization.

Bekeredjian et al. (2010) showed that mice carrying an oxygen-stable, inducible HIF-1 $\alpha$ (tetoff system) show an increased heart to body weight ratio after removal of doxycycline. Most interestingly this effect was fully reversible by restoring doxycycline to the drinking water and thus, restoring low HIF-1 $\alpha$ protein levels so that the heart to body weight ratio gradually decreased back to basal level.

The definite reasons for the induction of cardiomyopathy by HIF-1 remain still unclear. But it is very likely that it is a multifactorial process. The HIF-mediated metabolic switch to glyoclysis can drive the detrimental outcome of long-term HIF-1 $\alpha$ stabilization. Taegtmeyer et al. (1988) described that an adaptive metabolic switching from fatty acid to glucose metabolism can precede the development of hypertrophy (Taegtmeyer et al., 1988). The

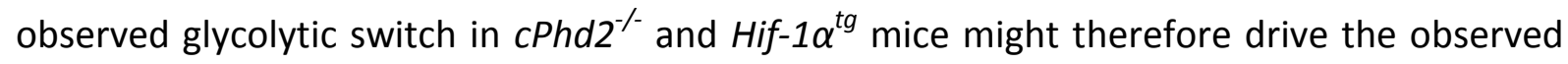
cardiac hypertrophy observed in aging mice. 


\section{Discussion}

After triggering cardiac hypertrophy by TAC surgery $\mathrm{CPhd2} 2^{-/-}$mice did not react differently than wt littermates (Hölscher et al., 2011, fig. 7), while Hif-1 $\alpha^{\text {tg }}$ mice showed severe signs of a dilated cardiomyopathy (Hölscher et al., 2012, fig. 5). The different results of $c P h d 2^{-/}$and $H i f-1 \alpha^{t g}$ to the pressure induced overload suggest that the severity of cardiomyopathy might be determined by the level of HIF-1 $\alpha$ stabilization.

These findings indicate that HIF-1 is indeed critically involved in the adaptation towards an increased mechanical load. In contrast to an acute ischemic insult, where HIF-mediated changes seem beneficial to withstand this situation, HIF-1 $\alpha$ stabilization seems to be inadequate in order to treat diseases related to elevated load.

In summary we can conclude that HIF-mediated changes in metabolism, apoptosis, calcium handling and capillary area are beneficial to withstand acute ischemia but that long-term stabilization of HIF-1 $\alpha$ has detrimental effects which are likely to be caused by various factors that have to be further elucidated. Thereby is seems that the severity of cardiomyopathy is determined by the level of HIF-1 $\alpha$ stabilization.

However, it remains possible that the threshold of HIF activity required for therapeutic effects (e.g. attenuation of outcome of myocardial infarction; red blood cell production in severe anemia) is lower than the threshold required for deleterious effects.

\section{$5.3 \mathrm{cPhd} 2 / 3^{\%-}$ mice}

Using the $\mathrm{CPhd} 2^{-/-}$mice we showed that PHD3 is significantly up-regulated as a consequence of a PHD2 knock-out (Hölscher et al., 2011, fig. 1). As PHD2 and PHD3 have partially redundant functions is it likely that the effects seen by the PHD2 knock-out are attenuated by the regulatory feedback up-regulation of PHD3. Subsequently we created cardiomyocytespecific PHD2/PHD3 double knock-out mice ( $\left(\mathrm{Phd} 2 / 3^{-}\right)$in order to see if the changes in e.g. metabolism and cardioprotection are more profound. $c P h d 2 / 3^{--}$mice were expected to have high levels of HIF-1 $\alpha$ protein as HIF-1 $\alpha$ does not get hydroxylated by PHD2 and PHD3 in these mice. Surprisingly these mice do only show modestly elevated HIF-1 $\alpha$ protein levels that do not exceed those of Hif- $1 \alpha^{t g}$ mice. The HIF-1 $\alpha$ mRNA levels are even diminished (chapter 4.2, fig. 1). Therefore, it is somewhat unlikely that the slightly elevated HIF-1 $\alpha$ protein levels are the main reason for the cardiomyopathy and the premature death of the knock-out mice. In further studies cardiomyocyte-specific PHD2/PHD3/HIF-1 $\alpha$ triple knock-out mice will be studied to determine the contribution of HIF-1 $\alpha$ to this phenotype. 


\section{Discussion}

Besides HIF-1 $\alpha$ the PHDs can also hydroxylate other substrates. A recently published paper (Yan et al., 2011) showed an activation of 5'adenosine-monophosphate-activated protein kinase (AMPK) by activation of the prolyl-hydroxylase oxygen-sensing signal cascade through inhibition of the PHDs by PHD2-RNAi or dimethyloxaloylglycine (DMOG), a cell-permeable 2OG (2-oxoglutarate) analog that is expected to act as a competitive inhibitor against all 2OG-dependent dioxygenases, in cardiomyocytes. AMPK plays an important role in cellular energy homeostasis; it can increase cellular energy levels by inhibiting anabolic energy consuming pathways (e.g. fatty acid synthesis) and stimulate energy producing, catabolic pathways (e.g. glucose transport).

One might speculate that AMPK might be activated in cardiomyocytes of $c P h d 2 / 3^{-/}$mice. In the cardiac hypertrophy associated with glycogen overload, both activating and inactivating mutations of AMPK in mice are associated with a marked cardiac hypertrophy (Dyck and Lopaschuk, 2006). Therefore it is tempting to speculate that besides HIF-1 $\alpha$ AMPK contributes to the observed phenotype in $\mathrm{cPhd} 2 / 3^{--}$mice. However it is likely that there will be a multitude of other factors that have to be determined in order to fully understand the molecular basis for the detrimental outcome of the double knock-out of PHD2 and PHD3 in cardiomyocytes of mice.

\subsection{PHD inhibitors as a therapeutic approach in acute myocardial infarction}

Patients with myocardial infarction (MI) are treated with stents or fibrolytic therapy to enable tissue reperfusion (Andersen et al., 2003). Patients that have survived an $\mathrm{MI}$ are still endangered to suffer from heart failure as their hearts are scarred and became enlarged as the heart tries to compensate for the insufficient pump function.

So far no therapy has been developed that directly aims to protect the heart muscle during the ischemic infarct. While the condition of the cells in the main area of the infarct region is not improvable it is of great interest to improve the situation for the cells in the hypoxic region surrounding the infarct area.

Since the discovery of HIF and the PHDs the HIF pathway has been exploited for therapeutic purposes. The use of PHD inhibitors is a promising new therapeutic option in treating diseases like myocardial infarction but also stroke, renal or liver injury or severe anemia.

Earlier it was shown by Natarajan et al. (2006) that normoxic activation of HIF-1 in the heart by PHD2 siRNA administration attenuates reperfusion injury via an iNOS-dependent 


\section{Discussion}

pathway. Since then, two novel orally active HIF-PHD inhibitors, GSK360A (Toronto Research Chemicals) and FG2216 (Fibrogen), were developed and are now being tested.

In a rat model of LAD ligation it has been shown that chronic treatment with GSK360A for 28 days prevented the progressive reduction in ejection fraction (Bao et al., 2010). In a different study (Philipp et al., 2006) it could be shown that treatment with FG2216 induces a significantly reduced heart and lung weight, improved left ventricular contractility and left ventricular ejection fraction without affecting infarct size in rats 30 days after myocardial infarction.

Despite these promising results further studies need to be conducted in order to determine the long-term consequences of these inhibitors. So far the effects of chronic PHD inhibition are largely unknown and existing data are only obtained from genetically modified knock-out mice. Furthermore the up to now available PHD inhibitors are not suitable to test PHD isoform-specific effects. Based on our results of the detrimental outcome of a cardiomyocyte-specific PHD2/PHD3 double knock-out mouse it seems indispensable to develop PHD inhibitors that inhibit specifically one isoform.

In rat heart-derived $\mathrm{H} 9 \mathrm{c} 2$ cells PHD3 silencing substantially ameliorates doxorubicin-induced apoptosis, whereas PHD1 or PHD2 knockdown did not significantly influence apoptosis. Thus, PHD3 is likely to be cardioprotective by reducing apoptosis in some heart disorders (Liu et al., 2010). Therefore it would be interesting to design a PHD3 specific inhibitor to determine if the apoptosis can be limited after an ischemic infarct.

Furthermore it would be interesting to design a PHD1 specific inhibitor as PHD1 knock-out mice show significant protection in a myocardial ischemia/reperfusion model (Adluri et al., 2011). But in contrast to cardiomyocyte-specific PHD2 knock-out mice which develop cardiomyopathy over time in the here presented study do PHD1 knockout mice survive without any cardiac defects until adulthood (Takeda et al., 2007). The reason for this difference might be that PHD1 loss induces HIF-1 $\alpha$ to a lesser extent than the loss of PHD2, so that there might be sufficient HIF to provide protection from ischemia but without activating other detrimental pathways.

Besides treatment of myocardial infarction PHD inhibitors are currently being tested for the treatment of anemia. Whereas treatment for tissue protection is based on short term application the treatment of anemia is designed to be a chronic treatment. Furthermore in tissue protection the treatment is based on the diversity of HIF target genes and their 


\section{Discussion}

potential to protect cells against consequences of hypoxia whereas in anemia treatment is based on a single target gene, erythropoietin. Phase 2 clinical trials in patients with chronic kidney disease are currently under way, studying the effect of oral PHD inhibitors as potential substitutes to EPO injection (Fibrogen, Press release 17.03.2011: Fibrogen announces initiation of phase 2B studies of FG-4592, an oral HIF proly hydroxylase inhibitor, for treatment of anemia in chronic kidney disease).

As the long-term stabilization of HIF-1 $\alpha$ seems to have detrimental long-term effects (Hölscher et al., 2012; Bekeredjian et al., 2010) PHD inhibitors will have to be carefully titrated to achieve beneficial effects without doing more harm than good.

\subsection{Summary and Outlook}

In this thesis several mouse models with different levels of HIF-1 $\alpha$ protein stabilization in cardiomyocytes were analyzed. It could be shown that HIF-1 $\alpha$ stabilization has a beneficial outcome in the setting of acute myocardial infarction. $c P h d 2^{-/}$and Hif- $1 \alpha^{\text {tg }}$ mice have a smaller area at risk six hours after the ligation of the left anterior descending artery and a better preserved heart function three, respectively four weeks after surgery (Hölscher et al., 2011; Kido et al., 2005). Likely due to the stabilization of HIF-1 $\alpha$ the vascular tone, cardiac metabolism, and the calcium handling of the cells are changed. The metabolism of $c P h d 2^{-/-}$ and Hif- $1 \alpha^{\text {tg }}$ mice is shifted towards glycolysis with a net increase in glucose uptake. Furthermore the calcium handling of isolated cardiomyocytes is, at least in Hif- $1 \alpha^{\text {tg }}$ mice, changed, leading to increased intracellular $\mathrm{Ca}^{2+}$-transients when stimulated at $0.5-4 \mathrm{~Hz}$. Although being beneficial in the setting of acute myocardial infarction, these changes lead in the long term to hypertrophy that goes along with heart failure. Still unclear is, however, if hypoxia is reason for or outcome of cardiac hypertrophy. To analyze if in a developing hypertrophy hypoxia leads to hypertrophy or if hypertrophy subsequently leads to hypoxia mice carrying the oxygen-dependent degradation domain in fusion with the firefly luciferase will be analyzed (Safran et al., 2005) in further studies. This fusion protein makes it possible to determine the bioluminescence which correlates with the stabilization of HIF-1 $\alpha$. In ODDluciferase mice the luciferase is continuously transcribed and translated in all cells, but is also rapidly degraded under normoxic conditions. Only in hypoxia does luciferase accumulate and, thus, by providing the substrate luciferin, regions of hypoxia within these 


\section{Discussion}

mice may be imaged. With the help of this mouse model the question what comes firsthypertrophy or hypoxia- can hopefully be answered.

In the future it will be worthwhile to look deeper into the consequences of cardiomyocytespecific HIF-1 $\alpha$ up-regulation. It will be interesting to know if the mitochondrial function is affected, and if so, if this leads to an improved or an impaired cellular respiration. Furthermore, it will be interesting to develop fibroblast-specific PHD2 knock-out mice to elaborate the differences and the similarities between cardiomyocyte- and fibroblastspecific PHD2 knock-out mice.

In this thesis the first unpublished results of cardiomyocyte-specific PHD2/PHD3 knock-out mice were described. These mice show a significant down-regulation of PHD2 and PHD3. Unexpectedly, these mice do not show excessively elevated HIF-1 $\alpha$ levels but instead they show a significant down-regulation of HIF-1 $\alpha$ mRNA and only a modest stabilization of HIF-1 $\alpha$ protein levels. $c P h d 2 / 3^{--}$mice develop a dilated cardiomyopathy already at a young age that finally leads to premature death. The main cause for this phenotype has to be further elucidated. Besides the HIF-1 $\alpha$ stabilization HIF-independent but PHD-dependent pathways are likely to be involved.

In summary, we could show that cardiomyocyte-specific HIF-1 $\alpha$ stabilization, mediated by a knock-out of PHD2, protects against acute myocardial infarction. Via the stabilization of HIF-1 $\alpha$ HIF target genes are transcriptional activated and thereby the calcium handling, the cell metabolism and the capillary structure are altered. These changes seem to cause cardiac hypertrophy over time.

In figure 3 a schematic overview demonstrating possible mechanisms involved in beneficial and detrimental effects of cardiac-specific HIF-1 $\alpha$ up-regulation are shown. 


\section{Discussion}

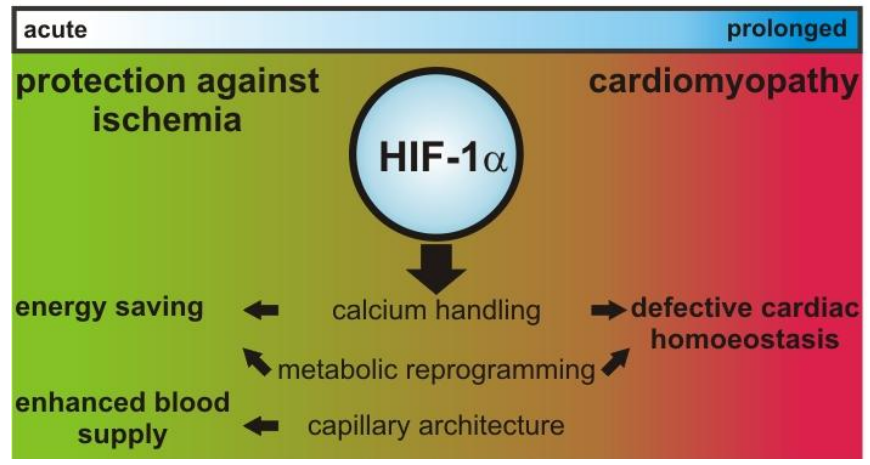

Hölscher et al., 2012

Fig.3 Schematical illustration of possible mechanisms that are involved in beneficial and detrimental effects of cardiac-specific HIF-1 $\alpha$ up-regulation.

Recapitulatory, it can be concluded from the here presented data that the HIF-mediated changes in metabolism, apoptosis, calcium handling and capillary area are on the one hand beneficial to withstand acute ischemia but are on the other hand, however, indicative for defective cardiac homeostasis in the failing heart. This has to be considered when designing PHD inhibiting drugs in the nearby future. 


\section{References}

\section{References}

Adluri RS, Thirunavukkarasu M, Dunna NR, Zhan L, Oriowo B, Takeda K, Sanchez JA, Otani H, Maulik G, Fong GH, Maulik N (2011). Disruption of Hypoxia-Inducible Transcription FactorProlyl Hydroxylase Domain-1 (PHD-1 ${ }^{-/-}$) Attenuates Ex Vivo Myocardial Ischemia/Reperfusion Injury Through Hypoxia-Inducible Factor-1 $\alpha$ Transcription Factor and Its Target Genes in Mice. Antioxidants \& Redox Signaling 15:1789-1797

Andersen HR, Nielsen TT, Rasmussen $K$, Thuesen L, Kelbaek $H$, Thayssen $P$, Abildgaard $U$, Pedersen F, Madsen JK, Grande P, Villadsen AB, Krusell LR, Haghfelt T, Lomholt P, Husted SE, Vigholt E, Kjaergard HK, Mortensen LS (2003). A Comparison of Coronary Angioplasty with Fibrinolytic Therapy in Acute Myocardial Infarction N Engl J Med 349:733-742

Appelhoff RJ, Tian YM, Raval RR, Turley H, Harris AL, Pugh CW, Ratcliffe PJ Gleadle JM (2004). Differential Function of the Prolyl Hydroxylases PHD1, PHD2, and PHD3 in the Regulation of Hypoxia-inducible Factor. J Biol Chem. 279:38458-38465

Asikainen TM and White CW (2007). HIF stabilizing agents: shotgun or scalpel? Am J Physiol Lung Cell Mol Physiol. 293:555-556

Bekeredjian R, Walton CB, MacCannell KA, Ecker J, Kruse F, Outten JT, Sutlcliffe D, Gerard RD, Bruick RK, Shohet RV (2010). Conditional HIF-1 $\alpha$ Expression Produces a Reversible Cardiomyopathy. PLoSONE 5(7):e11693

Benita Y, Kikuchi H, Smith AD, Zhang MQ, Chung DC, Xavier RJ (2009). An integrative genomics approach identifies Hypoxia Inducible Factor-1 (HIF-1)-target genes that form the core response to hypoxia. Nucleic Acids Research 37:4587-4602

Berra E, Benizri E, Ginouves A, Volmat V, Roux D, and Pouyssegur J (2003) HIF prolylhydroxylase 2 is the key oxygen sensor setting low steady-state levels of HIF-1alpha in normoxia. EMBO 22:4082-4090

Bohl S, Medway DJ, Schulz-Menger J, Schneider JE, Neubauer S, Lygate C (2009). Refined approach for quantification of in vivo ischemia-reperfusion injury in the mouse heart. Am J Physiol Heart Circ Physiol 297:H2054-2058.

Bruick RK and McKnight SLA (2001). Conserved Family of Prolyl-4-Hydroxylases That Modify HIF. Science 294:1337-1340

Cai Z, Manalo DJ, Wei G, Rodriguez ER, Fox-Talbot K, Lu H, Zweier JL, Semenza GL (2003). Hearts from rodents exposed to intermittent hypoxia or erythropoietin are protected against ischemia-reperfusion injury. Circulation 108:79-85

Chamboredon S, Ciais D, Desroches-Castan A, Savid P, Bonod F, Feige JJ, Cherradia N (2011). Hypoxia-inducible factor-1 $\alpha$ mRNA: a new target for destabilization by tristetraprolin in endothelial cells. Mol Biol Cell 18:3366-78 


\section{References}

Chan DA, Kawahara TLA, Sutphin PD, Chang HY, Chi JT, Giaccia AJ (2009). Tumor Vasculature Is Regulated by PHD2-Mediated Angiogenesis and Bone Marrow-Derived Cell Recruitment. Cancer Cell 15:527-538

Compernolle V, Brusselmans K, Acker T, Hoet P, Tjwa M, Beck H, PLaisance S, Dor $\mathrm{Y}$, Keshet E, Lupu F, Nemery B, Dewerchin M, Van Veldhoven P, Plate K, Moons L, Collen D, Carmeliet P (2002). Loss of HIF-2 $\alpha$ and inhibition of VEGF impair fetal lung maturation, whereas treatment with VEGF prevents fatal respiratory distress in premature mice. Nat Med 8:1329.

Cummins EP, Berra E, Comerford KM, Ginouves A, Fitzgerald KT, Seeballuck F, Godson C, Nielsen JE, Moynagh P, Pouyssegur J, Taylor CT (2006). Prolyl hydroxylase-1 negatively regulates IKB kinase- $\beta$, giving insight into hypoxia-induced NFkB activity. Proc Natl Acad Sci U S A. 103:18154-18159

Date T, Mochizuki S, Belanger AJ, Yamakawa M, Luo Z, Vincent KA, Cheng SH, Gregory RJ, Jiang $C$ (2005). Expression of constitutively stable hybrid hypoxia-inducible factor-1alpha protects cultured rat cardiomyocytes against simulated ischemia-reperfusion injury. Am J Physiol Cell Physiol 288:C314-C320

Dyck JRB and Lopaschuk GD (2006). AMPK alterations in cardiac physiology and pathology: enemy or ally? J Physiol 574:95-112

Ema M, Taya S, Yokotani N, Sogawa K, Matsuda Y, Fujii-Kuriyama Y (1997). A novel bHLH-PAS factor with close sequence similarity to hypoxia-inducible factor $1 \alpha$ regulates the VEGF expression and is potentially involved in lung and vascular development. Proc Natl Acad Sci USA 94:4273-4278

Erbel PJA, Card PB, Karakuzu O, Bruick RK, and Gardner KH (2003). Structural basis for PAS domain heterodimerization in the basic helix-loop-helix-PAS transcription factor hypoxiainducible factor. PNAS 100:15504-15509

Epstein ACR, Gleadle JM, McNeill LA, Hewitson KS,O'Rourke J, Mole DV, Mukherji M, Metzen E, Wilson MI, Dhanda A, Tian YM, Masson N, Hamilton DL, Jaakkola P, Barstead R, Hodgkin J, Maxwell PH, Pugh CW, Schofield J, Ratcliffe PJ (2011). C. elegans EGL-9 and Mammalian Homologs Define a Family of Dioxygenases that Regulate HIF by Prolyl Hydroxylation. Cell 107:43-54

Fibrogen press release (2011). FibroGen Announces Initiation of Phase 2b Studies of FG4592, an Oral HIF Prolyl Hydroxylase Inhibitor, for Treatment of Anemia in Chronic Kidney Disease

Flamme I, Frohlich T, von Reutern M, Kappel A, Damert A, and Risau W (1997). HRF, a putative basic helix-loop-helix-PAS-domain transcription factor is closely related to hypoxiainducible factor-1 alpha and developmentally expressed in blood vessels. Mech Dev 63:51-60

Goldberg MA, Dunning SP and Bunn HF (1988). Regulation of the erythropoietin gene: evidence that the oxygen sensor is a heme protein. Science 242:1412-1415 


\section{References}

Gu YZ, Moran SM, Hogenesch JB, Wartman L, Bradfield CA (1998). Molecular characterization and chromosomal localization of a third alpha-class hypoxia inducible factor subunit, HIF3alpha. Gene Expr 7:205-213

Hackett PH and Roach RC (2001). High-altitude illness. N Engl J Med. 345:107-114

Hara S, Hamada J, Kobayashi C, Kondo Y, Imura N (2001). Expression and Characterization of Hypoxia-Inducible Factor (HIF)-3 $\alpha$ in Human Kidney: Suppression of HIF-Mediated Gene Expression by HIF-3 $\alpha$. Biochem Biophys Res Commun 287:808-813

Hirsilä M, Koivunen P, Günzler V, Kivirikko KI, Myllyharju J (2003). Characterization of the Human Prolyl 4-Hydroxylases That Modify the Hypoxia-inducible Factor. J Biol Chem 278:30772-30780.

Hogenesch JB, Chan WK, Jackiw VH, Brown RC, Gu YZ, Pray-Grant M, Perdew GH, Bradfield CA (1997). Characterization of a subset of the basic-helix-loop-helix-PAS superfamily that interacts with components of the dioxin signaling pathway. J Biol Chem 272:8581-8593

Hölscher M, Schäfer K, Krull S, Farhat K, Hesse A, Silter M, Lin Y, Pichler BJ, Thistlethwaite P, El-Armouche A, Maier LS, Katschinski DM, Anke Zieseniss A (2012). Unfavourable consequences of chronic cardiac HIF-1 $\alpha$ stabilization. Cardiovasc Res. 94:77-86

Hölscher M, Silter M, Krull S, von Ahlen M, Hesse A, Schwartz P, Wielockx B, Breier G, Katschinski DM, Anke Zieseniss (2011). Cardiomyocyte-specific Prolyl-4-hydroxylase Domain 2 Knock Out Protects from Acute Myocardial Ischemic Injury. J Biol Chem 286:11185-11194

Hu CJ, Wang LY, Chodosh LA, Keith B, Simon MC (2003). Differential Roles of HypoxiaInducible Factor $1 \alpha$ (HIF-1 $\alpha$ ) and HIF-2 $\alpha$ in Hypoxic Gene Regulation. Mol Cell Biol 23:93619374

Iyer NV, Kotch LE, Agani F, Leung SW, Laughner E,Wenger RH, Gassmann M, Gearhart JD, Lawler AM, Yu AY, Semenza GL (1998). Cellular and developmental control of $\mathrm{O}_{2}$ homeostasis by hypoxia-inducible factor $1 \alpha$, Genes Dev 12:149-162

Kido M, Du L, Sullivan CC, Li X, Deutsch R, Jamieson SW, Thistlethwaite PA (2005). Hypoxia-inducible factor $1 \alpha$ reduces infarction and attenuates progression of cardiac dysfunction after myocardial infarction in the mouse. J Am Coll Cardiol 46:2116-2124

Koivunen P, Tiainen P, Hyvärinen J, Williams KE, Sormunen R, Klaus SJ, Kivirikko KI, and Myllyharju J (2007). An Endoplasmic Reticulum Transmembrane Prolyl 4-Hydroxylase Is Induced by Hypoxia and Acts on Hypoxia-inducible Factor $\alpha$. J Biol Chem 282:30544-30552

Laufs U, Maack C Und Böhm M (2008). Signaltransduktion bei adaptiven und maladaptiven kardialen Remodeling-Prozessen. Magazin Forschung 1/2008

Lieb ME, Menzies K, Moschella MC, Ni R, Taubman MB (2002) Mammalian EGLN genes have distinct patterns of mRNA expression and regulation. Biochem Cell Biol 80:421-426 


\section{References}

Liu Y, Huo Z, Yan B, Lin X, Zhou ZN, Liang X, Zhu W, Liang D, Li L, Liu Y, Zhao H, Sun Y, Chen YH (2010). Prolyl hydroxylase 3 interacts with $\mathrm{Bcl}-2$ to regulate doxorubicin-induced apoptosis in H9c2 cells. Biochem Biophys Res Commun 401:231-237

Loenarz C, Coleman ML, Boleininger A, Schierwater B, Holland PW, Ratcliffe PJ, Schofield CJ (2011). The hypoxia-inducible transcription factor pathway regulates oxygen sensing in the simplest animal, Trichoplax adhaerens. EMBO Rep 12:63-70

Lopaschuk GD, Ussher JR, Folmes CDL, Jaswal JS, Stanley WC (2010). Myocardial Fatty Acid Metabolism in Health and Disease. Physiol Rev 90:207-258

Mahon PC, Hirota K, and Semenza GL (2001). FIH-1: a novel protein that interacts with HIF$1 \alpha$ and VHL to mediate repression of HIF-1 transcriptional activity. Genes \& Dev 15:26752686

Maron BJ (2006). The 2006 American Heart Association Classification of Cardiomyopathies Is the GoldStandard. Circ Heart Fail 1:72-76

Maxwell PH, Wiesener MS, Chang GW, Clifford SC, Vaux EC, Cockman ME, Wykoff CC, Pugh CW, Maher ER, Ratcliffe PJ (199). The tumour suppressor protein VHL targets hypoxiainducible factors for oxygen-dependent proteolysis. Nature 399:271-275

McDonough MA, Li V, Flashman E, Chowdhury R, Mohr C, Liénard BMR, Zondlo J, Oldham NJ, Clifton IJ, Lewis J, McNeill LA, Kurzeja RJM, Hewitson KS, Yang E, Jordan S, Syed RS, Schofield CJ (2006). Cellular oxygen sensing: Crystal structure of hypoxia-inducible factor prolyl hydroxylase (PHD2). PNAS 103: 9814-9819

McMullen JR and Jennings GL (2007). Differences between pathological and physiological cardiac hypertrophy: novel therapeutic strategies to treat heart failure. Clin Exp Pharmacol Physiol 34:255-262

Metzen E, Berchner-Pfannschmidt U, Stengel P, Marxsen JH, Stolze I, Klinger M, Qi Huang W, Wotzlaw C, Hellwig-Bürgel T, Jelkmann W, Acker H, Fandrey J (2003). Intracellular localisation of human HIF-1 $\alpha$ hydroxylases: implications for oxygen sensing. J Cell Sci 116:1319-26.

Metzen E, Stiehl DP, Doege K, Marxsen JH, Hellwig-Bürgel T, Jelkmann W (2005). Regulation of the Prolyl Hydroxylase Domain Protein 2 (phd2/egln-1) gene: Identification of a functional hypoxia-responsive element. Biochem J 387:711-717

Mihl C, Dassen WRM, Kuipers H (2008). Cardiac remodelling: concentric versus eccentric hypertrophy in strength and endurance athletes. Neth Heart J 16:129-133.

Minamishima YA, Moslehi J, Bardeesy N, Cullen D, Bronson RT, Kaelin WG Jr (2008). Somatic inactivation of the PHD2 prolyl hydroxylase causes polycythemia and congestive heart failure. Blood 111:3236-44 


\section{References}

Natarajan R, Salloum FN, Fisher BJ, Kukreja RC, Fowler AA (2006). HIF-1 activation by prolyl 4-hyroxylase-2 gene silencing attenuates myocardial ischemia-reperfusion injury. Circ Res 98:133-140.

Oehme F, Ellinghaus $\mathrm{P}$, Kolkhof $\mathrm{P}$, Smith TJ, Ramakrishnan S, Hütter J, Schramm M, Flamme I (2002). Overexpression of $\mathrm{PH}-4$, a novel putative proline 4-hydroxylase, modulates activity of hypoxia-inducible transcription factors. Biochem Biophys Res Commun 296:343-349

Ortiz-Barahona A, Villar D, Pescador N, Amigo J, del Peso L (2010). Genome-wide identification of hypoxia-inducible factor binding sites and target genes by a probabilistic model integrating transcription-profiling data and in silico binding site prediction. Nuc Acids Res 38:2332-2345

Paralikar SJ and Paralikar JH (2010). High-altitude medicine. JH Indian J Occup Environ Med 14:6-12

Pescador N, Cuevas Y, Naranjo S, Alcaide M, Villar D, Land'Azuri MO, del Peso L (2005). Identification of a functional hypoxia-responsive element that regulates the expression of the egI nine homologue 3 (egln3/phd3) gene. Biochem J 390:189-197

Philipp S, Jürgensen JS, Fielitz J,Bernhardt WM, Weidemann A, Schiche A, Bernhard Pilz b, Dietz R, Regitz-Zagrosek V, Eckardt KU, Willenbrock R (2006). Stabilization of hypoxia inducible factor rather than modulation of collagen metabolism improves cardiac function after acute myocardial infarction in rats. Eur J Heart Fail 8:347-354

Reyes H, Reisz-Porszasz S, Hankinson O (1992). Identification of the Ah receptor nuclear translocator protein (Arnt) as a component of the DNA binding form of the Ah receptor. Science 256:1193-5.

Semenza GL. (1998). Hypoxia-inducible factor 1: master regulator of $\mathrm{O}_{2}$ homeostasis. Curr Opin Genet Dev 8:588-594

Sharp FR and Bernaudin M (2004). HIF1 and Oxygen Sensing in the Brain. Nat Rev Neurosci. 5:437-48.

Silter M, Kögler H, Zieseniss A, Wilting J, Schafer K, Toischer K, Rokita AG, Breves G, Maier LS, Katschinski DM (2010). Impaired Ca2+-handling in HIF-1 $\alpha^{+/-}$mice as a consequence of pressure overload. Pflugers Arch 459:569-577.

Patten RD, Aronovitz MJ, Deras-Mejia L, Pandian NG, Hanak GG, Smith JJ, Mendelsohn ME, Kosntam MA (1998). Ventricular remodeling in a mouse model of myocardial infarction. Am J Phys 274:H1812-1820.

Safran M, Kim WY, O’Connell F, Flippin L, GünzlerV, Horner JW, DePinho RA, Kaelin, Jr. WG (2006). Mouse model for noninvasive imaging of HIF prolyl hydroxylase activity: Assessment of an oral agent that stimulates erythropoietin production. PNAS 103:105-110 


\section{References}

Sano M, Minamino T, Toko H, Miyauchi H, Orimo M, Qin $\mathrm{Y}$, Akazawa H, Tateno K, Kayama $\mathrm{Y}$, Harada M, Shimizu I, Asahara T, Hamada H, Tomita S, Molkentin JD, Zou Y, Komuro I (2007). p53-induced inhibition of Hif-1 causes cardiac dysfunction during pressure overload. Nature 446:444-448

Stanley WC, Recchia FA, Lopaschuk GD (2005). Myocardial Substrate Metabolism in the Normal and Failing Heart. Physiol Rev 85:1093-1129

Taegtmeyer $\mathrm{H}$ and Overturf ML (1988). Effects of moderate hypertension on cardiac function and metabolism in the rabbit. Hypertension 11:416-26

Takeda K, Cowan A, Fong GH (2007). Essential Role for Prolyl Hydroxylase Domain Protein 2 in Oxygen Homeostasis of the Adult Vascular System. Circulation 116:774-781

Takeda K, Ho VC, Takeda H, Duan LJ, Nagy A, Fong GH (2006). Placental but Not Heart Defects Are Associated with Elevated Hypoxia-Inducible Factor $\alpha$ Levels in Mice Lacking Prolyl Hydroxylase Domain Protein 2. Mol Cell Biol 26:8336-8346

Tekin S, Dursun D, Xi L (2010). Hypoxia inducible factor 1 (HIF-1) and cardioprotection. Acta Pharm Sinic 31: 1085-1094

Tian H, Hammer RE, Matsumoto AM, Russell DW, McKnight SL (1998). The hypoxiaresponsive transcription factor EPAS1 is essential for catecholamine homeostasis and protection against heart failure during embryonic development. Genes Dev 12:3320-3324

Tian H, McKnight SL, Russell DW (1997). Endothelial PAS domain protein 1 (EPAS1), a transcription factor selectively expressed in endothelial cells. Genes Dev 11:72-82

Tian J, Yan Z, Wu Y, Zhang SL, Wang K, Ma XR, Guo L, Wang J, Zuo L, Liu JY, Quan L, Liu HR (2010). Inhibition of iNOS protects endothelial-dependent vasodilation in aged rats. Acta Pharm Sinic 31:1324-1328

Toischer K, Rokita AG, Unsöld B, Zhu W, MD, Kararigas G, Sossalla S, Reuter SP Becker A, Teucher N, Seidler T, Grebe C, Preuß L, Gupta SN, Schmidt K, Lehnart SE, Krüger M, Linke WA, Backs J, Regitz-Zagrosek V, Schäfer K, Field LJ, Maier LS, Hasenfuss G (2010). Differential Cardiac Remodeling in Preload Versus Afterload. Circulation 122: 993-1003

Warnecke C, Griethe W, Weidemann A, Jürgensen JS, Willam C, Bachmann S, Ivashchenko Y, Wagner I, Frei U, Wiesener M, Eckardt KU (2003). Activation of the hypoxia-inducible factorpathway and stimulation of angiogenesis by application of prolyl hydroxylase inhibitors. Faseb J 17:1186-8.

Yan L, Colandrea VJ, Hale JJ (2010). Prolyl hydroxylase domain-containing protein inhibitors as stabilizers of hypoxia-inducible factor: small molecule-based therapeutics for anemia. Expert Opin Ther Pat 20:1219-1245 


\section{References}

Yan H, Zhang DX, Shi X, Zhang Q, Huang YS (2011). Activation of the Prolyl-hydroxylase oxygen-sensing signal cascade leads to AMPK activation in cardiomyocytes. J Cell Mol Med doi: 10.1111/j.1582-4934.2011.01500.x

Zhang H, Bosch-Marce M, Shimoda LA, Tan YS, Baek JH, Wesley JB, Gonzalez FJ, and Gregg L. Semenza GL (2008). Mitochondrial Autophagy Is an HIF-1-dependent Adaptive Metabolic Response to Hypoxia. J Biol Chem 283:10892-10903 


\section{Acknowledgments}

\section{DANKE sagen möchte ich an dieser Stelle...}

... allen, die zum Gelingen dieser Arbeit beigetragen haben.

... Frau Prof. Dr. Dörthe M. Katschinski, die mir die Möglichkeit gab an diesem spannenden Thema zu arbeiten und mich mit vielen fachlichen Ratschlägen unterstützt hat. Zudem gab sie mir die Möglichkeit, meine Forschungsergebnisse auf mehreren nationalen und internationalen Tagungen präsentieren zu können.

... Dr. Anke Zieseniß. Danke für die tolle Betreuung und die wertvollen Tipps, die zum guten Gelingen dieser Arbeit beigetragen haben.

... Frau Prof. Dr. F. Alves und Frau Prof. Dr. S. Pöggeler für die freundliche Übernahme des Referates und Koreferates dieser Arbeit und ihre stets wertvollen Anregungen bei meinen Thesis Committees.

... Frau Prof. Dr. B. Schwappach, Frau Prof. Dr. Dr. H. Ehrenreich und Herrn Dr. V. Nikolaev für die sofortige Bereitschaft als Prüfer zur Verfügung zu stehen.

...Sabine für etliche qPCRs, Gewebeschnitte, Färbungen, etc...

...Monique, die mich geduldig in die mir bis dahin unbekannte Welt der "Mausarbeit“ eingearbeitet hat.

...Annette für unzählige Genotypisierungen.

...allen aktuellen und ehemaligen Mitarbeitern der AG Katschinski... Katja, Marieke, Sabine, Amke, Anke, Sabine, Melanie, Monique, Pia, Liza, Cordula, Ulrike, Jaco, Moritz und Annette für die tolle Atmosphäre und eure stete Hilfsbereitschaft und wertvollen Tipps. Ohne euch wäre die Laborzeit nur halb so toll gewesen...

... Frau Dr. Sarah Kimmina sowie den Mitarbeitern der Zentralen Tierexperimentellen Einrichtung der UMG für die freundliche Zusammenarbeit.

... allen, die mit mir die ein oder andere Stunde im OP3 zugebracht haben.

... AG Schäfer, insbesondere Frau Prof. Dr. K. Schäfer und Anika für die tolle Kooperation bei den Hif- $1 \alpha^{\text {tg }}$ Mäusen.

... AG Maier, insbesondere Felicia, für die Calciummessungen.

... meiner Familie, die mich immer unterstützt hat.

... Sven. Einfach danke für alles... 


\section{Curriculum vitae}

Name

Date of birth

Place of birth

August 1993-July 2002

July 2002-August 2003

October 2003-July 2008

June 2009-June 2012
Marion Hölscher

22.09.1982

Paderborn, Germany

Pelizaeus Secondary School, Paderborn

Au pair in Park City, Utah, USA

Diploma Biology, Justus-Liebig University, Gießen, Germany

Thesis title: "Kleine nicht-codierende RNAs in S.meliloti $1021^{\prime \prime}$

PhD student at the Department of Cardiovascular Physiology, Georg-August University, Göttingen, Germany

Thesis title: "Cardiac functions of the cellular oxygen sensors prolyl-4-hydroxylase domain enzymes 2 and 3"

\section{Publications}

Björn Voss, Marion Hölscher, Birgit Baumgarth, Andreas Kalbfleisch, Ceren Kaya, Wolfgang R. Hess, Anke Becker, Elena Evguenieva-Hackenberg (2009)

Expression of small RNAs in Rhizobiales and protection of a small RNA and its degradation products by $\mathrm{Hfq}$ in Sinorhizobium meliloti

Biochem Biophys Res Commun 390:331-336

Marion Hölscher, Monique Silter, Sabine Krull, Melanie von Ahlen, Amke Hesse, Peter Schwartz, Ben Wielockx, Georg Breier, Dörthe M. Katschinski, Anke Zieseniss (2011)

Cardiomyocyte-specific Prolyl-4-hydroxylase domain 2 knock out protects from acute myocardial ischaemic injury

J Biol Chem 286:11185-11194

Marion Hölscher, Katrin Schäfer, Sabine Krull, Katja Farhat, Amke Hesse, Monique Silter, Yun Lin, Bernd J. Pichler, Patricia Thistlethwaite, Ali El Armouche, Lars S. Maier, Dörthe M. Katschinski*, Anke Zieseniss* (*both authors contributed equally) (2012)

Unfavourable consequences of chronic cardiac HIF-1 $\alpha$ stabilization

Cardiovasc Res 94:77-86 


\section{Conferences}

18.-19.10.2010 2nd EUGENEHEART PhD Student Exchange Meeting London, England

poster presentation

08.-12.01.2011 Oxygen 2011

Davos, Switzerland

poster presentation

12.-17.02.2012 Advances in Hypoxic Signaling: From Bench to Bedside Banff, Alberta, Canada

poster presentation

22.-25.03.2012 $91^{\text {st }}$ Annual Meeting of the Deutsche Physiologische Gesellschaft Dresden, Germany poster presentation (poster prize award) 